\title{
From Molecular Mechanisms to Clinical Management of Antineoplastic Drug-Induced Cardiovascular Toxicity: A Translational Overview
}

\author{
Carlo Gabriele Tocchetti, ${ }^{1}$ Christian Cadeddu, Daniela Di Lisi, Saveria Femminò, ${ }^{3}$ Rosalinda Madonna,,6 \\ Donato Mele,, Ines Monte, Giuseppina Novo, Claudia Penna, Alessia Pepe, Paolo Spallarossa, ${ }^{4}$ \\ Gilda Varricchi, ${ }^{1,11}$ Concetta Zito, ${ }^{12}$ Pasquale Pagliaro,, ${ }^{4}$ and Giuseppe Mercuro, ${ }^{2, *}$
}

\begin{abstract}
Significance: Antineoplastic therapies have significantly improved the prognosis of oncology patients. However, these treatments can bring to a higher incidence of side-effects, including the worrying cardiovascular toxicity (CTX).

Recent Advances: Substantial evidence indicates multiple mechanisms of CTX, with redox mechanisms playing a key role. Recent data singled out mitochondria as key targets for antineoplastic drug-induced CTX; understanding the underlying mechanisms is, therefore, crucial for effective cardioprotection, without compromising the efficacy of anti-cancer treatments.

Critical Issues: CTX can occur within a few days or many years after treatment. Type I CTX is associated with irreversible cardiac cell injury, and it is typically caused by anthracyclines and traditional chemotherapeutics. Type II CTX is generally caused by novel biologics and more targeted drugs, and it is associated with reversible myocardial dysfunction. Therefore, patients undergoing anti-cancer treatments should be closely monitored, and patients at risk of CTX should be identified before beginning treatment to reduce CTX-related morbidity. Future Directions: Genetic profiling of clinical risk factors and an integrated approach using molecular, imaging, and clinical data may allow the recognition of patients who are at a high risk of developing chemotherapy-related CTX, and it may suggest methodologies to limit damage in a wider range of patients. The involvement of redox mechanisms in cancer biology and anticancer treatments is a very active field of research. Further investigations will be necessary to uncover the hallmarks of cancer from a redox perspective and to develop more efficacious antineoplastic therapies that also spare the cardiovascular system. Antioxid. Redox Signal. 30, 2110-2153.
\end{abstract}

Keywords: chemotherapy, ErbB2 inhibitors, vascular endothelial growth factor, tyrosine kinase inhibitors, oxidative/nitrosative stress, cancer immunotherapy

Reviewing Editors: Gregory Bellot, Jozef Dulak, Hasan Mukhtar, Pasquale Pignatelli, Des Richardson, Martin Sterba, Yuchiro Suzuki, and Oren Tirosh

${ }^{1}$ Department of Translational Medical Sciences, Federico II University, Naples, Italy.

${ }^{2}$ Department of Medical Sciences and Public Health, University of Cagliari, Cagliari, Italy.

${ }^{3}$ Biomedical Department of Internal Medicine, University of Palermo, Palermo, Italy.

${ }^{4}$ Department of Clinical and Biological Sciences, University of Turin, Turin, Italy.

${ }^{5}$ Center of Aging Sciences and Translational Medicine - CESI-MeT, "G. d'Annunzio" University, Chieti, Italy.

${ }^{6}$ Department of Internal Medicine, The Texas Heart Institute and Center for Cardiovascular Biology and Atherosclerosis Research, The University of Texas Health Science Center at Houston, Houston, Texas.

${ }^{7}$ Cardiology Unit, Emergency Department, University Hospital of Ferrara, Ferrara, Italy.

${ }^{8}$ Department of General Surgery and Medical-Surgery Specialities, University of Catania, Catania, Italy.

${ }^{9}$ U.O.C. Magnetic Resonance Imaging, Fondazione Toscana G. Monasterio C.N.R., Pisa, Italy.

${ }^{10}$ Clinic of Cardiovascular Diseases, IRCCS San Martino IST, Genova, Italy.

${ }^{11}$ Center for Basic and Clinical Immunology Research (CISI) - Federico II University, Naples, Italy.

${ }^{12}$ Division of Cardiology, Clinical and Experimental Department of Medicine and Pharmacology, Policlinico "G. Martino" University of Messina, Messina, Italy.

*These authors share senior authorship. 
Table of Contents

I. Introduction: The Clinical Problem of Antineoplastic Drug Cardiovascular Toxicity 2112

II. Anthracyclines 2113

A. Adverse effects

B. Mechanisms of adverse effects

C. Ways to reduce CV toxicity 2114

III. ErbB2 Inhibitors

A. Adverse effects

B. Mechanisms of adverse effects

C. Ways to reduce CV toxicity 2116

IV. VEGF Inhibitors and Multi-Targeted Kinase Inhibitors 2116

A. Adverse events

B. Mechanisms of adverse effects 2117

V. TKIs and Anti-BCR-abl Agents 2118

A. Imatinib adverse events $\quad 2119$

B. Mechanisms of imatinib-induced adverse events 2119

C. Nilotinib adverse events

D. Mechanisms of nilotinib-induced adverse events 2119

E. Dasatinib adverse events 2120

F. Mechanisms of dasatinib-induced adverse events 2120

G. Bosutinib adverse events 2120

$\begin{array}{ll}\text { H. Ponatinib adverse events } & 2120\end{array}$

VI. Taxanes 2121

A. Adverse effects 2121

B. Mechanisms of adverse effects 2121

C. Ways to reduce CV toxicity 2121

VII. Cancer Immunotherapy 2121

A. Immune checkpoint inhibitors 2121

B. CTLA-4 2121

C. PD-1/PD-L1 pathway 2121

D. Combination of checkpoint inhibitors 2122

E. Checkpoint inhibitors and immune-related adverse events 2122

VIII. Antimetabolites 2122

A. Adverse effects $\quad 2122$

B. Mechanism of adverse effects 2123

IX. Proteasome Inhibitors 2123

A. Adverse effects

B. Mechanisms of adverse effects 2124

C. Ways to reduce CV toxicity 2124

X. The Importance of Mechanisms Such as Oxidative and Nitrosative Stress in CV Toxicity: An Overview 2124

A. Drugs with antioxidant properties

1. $\beta$ blockers: Anti-adrenergic and antioxidant drugs 2127

2. The redox role of renin-angiotensin-aldosterone system antagonists: ACE-Is and ARBs 2128

B. Novel strategies in cardioprotection against ANT-induced CTX 2129

C. Nonpharmacologic strategies 2130

D. Biomarkers of oxidative/nitrosative CTX 2130

XI. Early Detection, Monitoring, and Management of Heart and Vascular Toxicities 2130

A. Cardioncology: an expanding science for an expanding problem 2130

B. Diagnostic tools for CTX detection 2130

C. LVEF and emerging modalities for assessing myocardial damage: some clarifying points 2131

D. Cardiac biomarkers

E. Detection of vascular complications: old and new strategies 2132

F. Timing of clinical evaluation and follow-up: the importance of risk factors assessment 2133

G. Drug-specific timing for CTX monitoring and treatment 2133

$\begin{array}{lr}\text { 1. ANTs } & 2133\end{array}$

$\begin{array}{lr}\text { 2. Anti-ErbB2 } & 2134\end{array}$

\begin{tabular}{lr}
3. & 2134 \\
\hline
\end{tabular}

XII. Conclusions and Perspectives 2134 


\section{Introduction: The Clinical Problem of Antineoplastic Drug Cardiovascular Toxicity}

D URING THE PREVIOUS YEARS, the prognosis of cancer was greatly enhanced by advancements in antitumoral therapeutic protocols; many types of malignancies can now be cured or maintained in remission for a long time, allowing patients to live the rest of their lives in remission from cancer $(21,132$, 173, 266, 392). Unfortunately, antitumoral treatments exert some adverse side-effects (Table 1). The cardiovascular (CV) system can be negatively affected by such therapies, and this is especially true in the so-called long-term cancer survivors, since the likelihood that cardiac side-effects of antitumoral treatments become the main health problem after tumor elimination increases with survival $(250,287,429,432)$.

The most common CV complications of antineoplastic therapies include vasospastic and thromboembolic ischemia, arterial hypertension, dysrhythmia, and left ventricular (LV) dysfunction, leading to heart failure (HF) $(25,204,376,429$, 432). Cardiac dysfunction caused by anthracyclines (ANTs) has long been known as the main form of anti-cancer druginduced cardiotoxicity (CTX) (91-94), with production of reactive oxygen species (ROS) and reactive nitrogen species (RNS) being considered main cytotoxic mechanisms (see section $X$ for details). In the past decades, new biologic anticancer drugs, such as intracellular signaling inhibitors, were increasingly used. These molecules may also be cardiotoxic, since they block pathways that are major modulators of myocardial function, especially under conditions of cardiac stress, such as hypertension or hypertrophy (376), with mechanisms of action that often involve redox signaling as well. As an example, drugs that target the human epidermal growth factor receptor $2(H E R / E r b B 2)$ and the vascular endothelial growth factor $(V E G F)$ exert a considerable adverse

Table 1. Drugs: Indications and Associated Types of Cardiovascular Toxicity

Class/drug Indication Toxicity

Anthracyclines

Doxorubicin, daunorubicin, epirubicin

Anti-ErbB2

Trastuzumab, lapatinib, pertuzumab

Breast cancer, leukemia, lymphoma, ovarian cancer, sarcoma

Breast cancer, gastric cancer

Vascular endothelial growth factor

inhibitors and multi-targeted

kinase inhibitors

Bevacizumab, sunitinib, sorafenib, regorafenib, pazopanib, axitinib, vandetanib

Gastrointestinal cancer, hepatocellular carcinoma, renal cell carcinoma,

Tyrosine kinase inhibitors and anti-BCR-abl agents

Imatinib, nilotinib, dasatinib, bosutinib, ponatinib

Chronic myeloid leukemia

Taxanes

Docetaxel, paclitaxel

Cancer Immunotherapies

Ipilimumab, nivolumab,

pembrolizumab, atezolizumab

Antimetabolites

Fluorouracil (5-FU), capecitabine, gemcitabine

Proteasome inhibitors

Bortezomib; carfilzomib, ixazomib, delanzomib, oprozomib, and marizomib
Breast cancer, colorectal cancer, nonsmall cell lung cancer, and ovarian cancers

Metastatic melanoma

Metastatic nonsmall cell lung cancer (NSCLC)

Advanced renal cell carcinoma

Classical Hodgkin lymphoma

Advanced urothelial carcinoma

Gastrointestinal, breast, head, neck, and pancreatic cancer

Multiple myeloma and other hematologic conditions (amyloidosis, non-Hodgkin lymphoma)
LV dysfunction/HF (2\%-48\% incidence)

LV dysfunction $(0.2 \%-20.1 \%$ incidence $)$

LV dysfunction (2.7\%-19\% incidence), Hypertension $(15 \%-44 \%$ incidence), Vascular damage $(1.4 \%-3.8 \%$ incidence)

Edema, cardiac dysfunction ( $0.2 \%-4 \%$ incidence), QTc prolongation $(2 \%-40.5 \%$ incidence)

Bradycardia, LV dysfunction, ischemia $(0.3 \%-1.7 \%$ incidence)

Immune myocarditis $(0.01 \%-0.27 \%$ incidence $)$

Coronary spasms/ischemia (7\%-18\% incidence)

LV dysfunction $(2 \%-25 \%$ incidence)

Modified from Suter and Ewer (376) and Zamorano et al. (432)

ErbB2, human epidermal growth factor receptor 2 (HER2); HF, heart failure; LV, left ventricular. 
effect on myocardial function via different mechanisms, based on the role of the proteins inhibited. The toxicity produced by biologic drugs seems to be due to mechanisms other than cardiomyocyte disruption, is most often reversible with discontinuation of the drugs, and has been classified as type II CTX $(93,94)$. On the other hand, ANTs produce a form of cardiac dysfunction that is typically irreversible, termed type I CTX, and that is characterized by evident ultrastructural myocardial abnormalities $(93,94)$. Of note, these two CTX paradigms may overlap. One paradigmatic example is the ErbB2 receptor inhibitor trastuzumab, which can cause irreversible LV dysfunction in patients previously treated with ANTs $(376,432)$, with the neuregulin/ErbB2 pathway that seems to modulate the increase in ROS-caused ANTs (390).

In this article, we address the main cellular and molecular mechanisms and pathophysiologic and clinical characteristics of antineoplastic drug-related CTX, since only a comprehensive assessment of this phenomenon can provide important hints to predict, treat, and prevent it. Special emphasis is placed on LV dysfunction and HF, in consideration of their clinical and social burden $(25,125)$, with updated insights regarding the role of oxidative damage, a mechanism that appears to have a major role in antineoplastic druginduced CTX $(412,432)$. Whenever appropriate, we divided the various sections into three categories (i) adverse effects, (ii) mechanisms of adverse effects, and (iii) ways to reduce CV toxicity.

\section{Anthracyclines}

Among the drugs with a greater cardiotoxic potential, ANTs are good representatives of the type I CTX paradigm.

ANTs are widely used and effective antineoplastic drugs, which are indicated for the therapy of many kinds of cancers, including lymphomas, leukemias, and sarcomas, and for both early and advanced breast cancer. However, these drugs have been recognized as cardiotoxic since the 1960s (384).

\section{A. Adverse effects}

ANT-induced CTX can manifest as a sort of cardiomyopathy, referred to as ANT-induced cardiomyopathy, leading to HF, which limits the usability of the drugs, with important consequences for managing malignancies. ANT-related CTX represents a significant clinical burden, producing LV dysfunction in a maximum of $9 \%$ of cases in a recent large prospective study (35).

\section{B. Mechanisms of adverse effects}

ANT-related CTX mechanisms are derived from a combination of several cellular and molecular alterations, leading to myocardial damage and dysfunction (Fig. 1). ANTinduced CTX is still not completely understood, though new insights on molecular mechanisms have been elucidated in recent years $(373,432,435)$.

The role of redox stress, resulting from an overproduction of ROS/RNS, in ANT-related CTX is undisputed (246, 247, 353,434 ), although mechanisms are much less clear (see section X for further details). ROS and RNS may be not only either direct or indirect inducers of the cardiac injury but also triggers, byproducts of the injury, or end-products arising

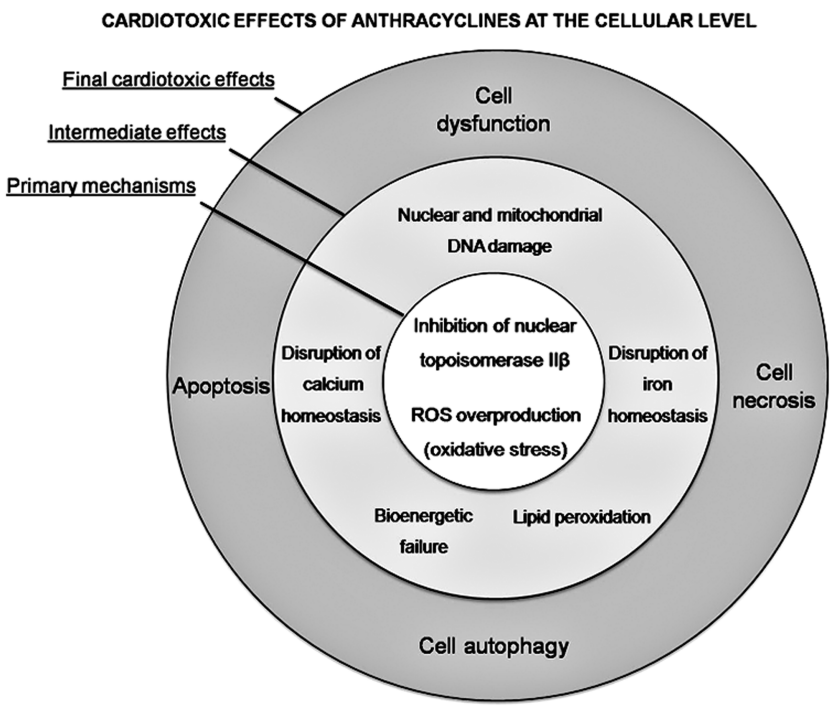

FIG. 1. Concentric representation of cardiotoxic effects of anthracyclines. ROS, reactive oxygen species.

from the injury itself. In addition, severity of oxidative/ nitrosative stress often does not correspond well with the severity of the injury. This is connected to acceptance and explanation of poor efficacy of different antioxidants under relevant conditions. Recent data suggest that ANT-induced CTX is also strictly related to ANT interaction with topoisomerase 2 (Top2) $\beta$ in cardiomyocytes (229). Two types of Top 2 enzymes are targeted by ANTs: Top $2-\alpha$ and Top $2 \beta$. The former is present in rapidly dividing cells, such as cancer cells, and forms the ternary Top2-doxorubicin-DNA cleavage complex, inducing cell apoptosis, whereas Top $2 \beta$ is present in human cardiomyocytes, forming the Top $2 \beta$-doxorubicinDNA complex, which causes DNA double-strand breaks and transcriptome modifications, also leading to cell apoptosis (434). A seminal paper confirmed the crucial role of the Top2 $\beta$ gene in an animal model, demonstrating that cardiomyocyte-specific deletion of the Top $2 \beta$ gene is protective against ANT-induced damage (434). The DNA break caused by doxorubicin binding to Top $2 \beta$ can, therefore, stimulate the DNA injury response. Consequently, the tumor suppressor protein $\mathrm{p} 53$, a fundamental enzyme for activating DNA repair proteins, can be induced. Unfortunately, p53 can also cause defective mitochondria biogenesis and metabolic failure by suppressing genes involved in organelle biogenesis, such as peroxisome proliferator-activated receptor gamma coactivator 1-alpha, and alteration of oxidative phosphorylation (434). Intriguingly, the metabolic perturbations induced by doxorubicin-activated p53 are responsible for altered autophagy, a process that is necessary for the normal recycling of dysfunctional mitochondria. Consequently, doxorubicindamaged mitochondria accumulate in the cardiomyocytes, resulting in enhanced ROS/RNS generation and, ultimately, cell death. Recent observations in p53-null mice found a smaller impairment in cardiac functional reserve after ANT treatment, supporting this hypothesis (157). Interestingly, in these mice, mitochondrial and LV function were maintained with increasing age, suggesting that p53-mediated inhibition of autophagy may play a role in all forms of cardiac dysfunction, not just doxorubicin-induced cardiomyopathy (157). 
Apart from p53, doxorubicin may also induce the mitogenactivated protein kinase (MAPK) pathway via ROS- and $\mathrm{Ca}^{2+}$-dependent mechanisms (437). Importantly, extracellular signal-regulated kinases (ERKs), members of the MAPK family, may protect myocytes from apoptosis, whereas p38 MAPK induces death of cardiomyocytes (437). More studies are needed to elucidate the role of such kinases and of other less-characterized signaling pathways in ANT-induced cardiotoxicity. However, these data confirm that oxidative reactions, at the basis of ANT-induced LV dysfunction, are involved in most types of HF. Therefore, timely innovative pharmacological strategies that interfere with specific molecules involved in heart dysfunction (e.g., p53) may represent a potential common approach in limiting HF occurrence $(250,341)$.

ANT-alcohol metabolites also play a pivotal role in inducing cellular injury and CTX via iron-dependent and -independent mechanisms. In fact, these metabolites disrupt iron and calcium homeostasis and, ultimately, lead to intracellular $\mathrm{Ca}^{2+}$ overload. Calcium overload has also been related to increased calpain proteolytic activity, which leads to cellular disarray and sarcomere disruption, resulting in sarcopenia (220). In addition, the interaction of ANTs with critical signaling pathways and with the activity of transcription factors may also explain sarcopenia, which derives from the limitation of sarcomere protein synthesis (165).

Mitochondrial activity has a central role in ANT-induced CTX $(257,258)$. The presence of doxorubicin in the mitochondrion, due to a high affinity for the mitochondrial phospholipid cardiolipin, negatively affects its function, stimulating ROS/RNS production, inhibiting oxidative phosphorylation, and causing mitochondrial DNA damage (300), with a consequent progressive reduction of energy production, leading to cell dysfunction (210). ANTs also appear to be responsible for mitochondrial calcium accumulation (300), leading to mitochondrial membrane injury. The mitochondrial pathway is an additional mechanism that is responsible for cellular intrinsic apoptosis. This involves elements of the outer mitochondrial membrane, including Bax and Bak, and activates cytochrome C and caspase, among others (257).

Some researchers have hypothesized that the loss of iron homeostasis and the $\mathrm{Ca}^{2+}$ overload caused by ANT alcohol metabolites, impairing cardiomyocyte energy and redox balance, could be sufficient to induce significant myocardial dysfunction, and that, in addition, cardiac injury could be increased by an apoptotic loss of cardiomyocytes triggered by ROS and $\mathrm{Fe}^{2+}(257,258)$. ANTs can also affect cardiac progenitor cells, hampering the regeneration capabilities of cardiac tissues after myocardial damage $(158,289)$. The controversy on the role of cardiac stem cells is discussed in the section XII.

Finally, it should be noted that several factors can favor ANT-induced CTX, and this may explain the individual variability in CTX occurrence $(246,247)$ (Fig. 2). The so-called multiple-hit hypothesis considers a late onset of CTX due to pharmacological and nonpharmacological subsequent injury. Therefore, strategies favoring cardiac adaptation to various stressors are crucial after ANT therapy (244). Of course, a better understanding of the molecular mechanisms of ANTrelated CTX is essential to choose the best strategies to prevent and treat CTX $(33,231,232,345,408)$.

\section{Ways to reduce $\mathrm{CV}$ toxicity}

Several approaches have been proposed to reduce ANT cardiotoxicity, including angiotensin-converting-enzyme inhibitors (ACE-Is), $\beta$ blockers, Doxrazoxane, phosphodiesterase-5 (PDE-5) inhibitors, Ranolazine and Statins, as well as nutritional supplementation and exercise training. The way the various approaches may reduce CV toxicity by ANT is treated in sections $\mathrm{X}$ and XI.

\section{ErbB2 Inhibitors}

ErbB2 (also called HER2) is a member of the human epidermal growth factor receptor family, which also includes ErbB1, ErbB3, and ErbB4. When bound by their ligands, these transmembrane receptors homodimerize or heterodimerize and are trans-phosphorylized, thus initiating several cellular responses. Until now, no specific ligand for ErbB2 has been identified, and the protein is believed to act as a dimerization partner of the other ErbBs (107). Importantly, ErbB2 is overexpressed in $\sim 30 \%$ of breast cancer cases, and it can then interact spontaneously with other ErbBs independently from ligand stimulation, thus triggering signaling pathways that stimulate tumor growth and survival (361).

\section{PREDISPOSING FACTORS ACCOUNTING FOR INDIVIDUAL VARIABILITY OF ANTHRACYCLINE CARDIOTOXICITY}

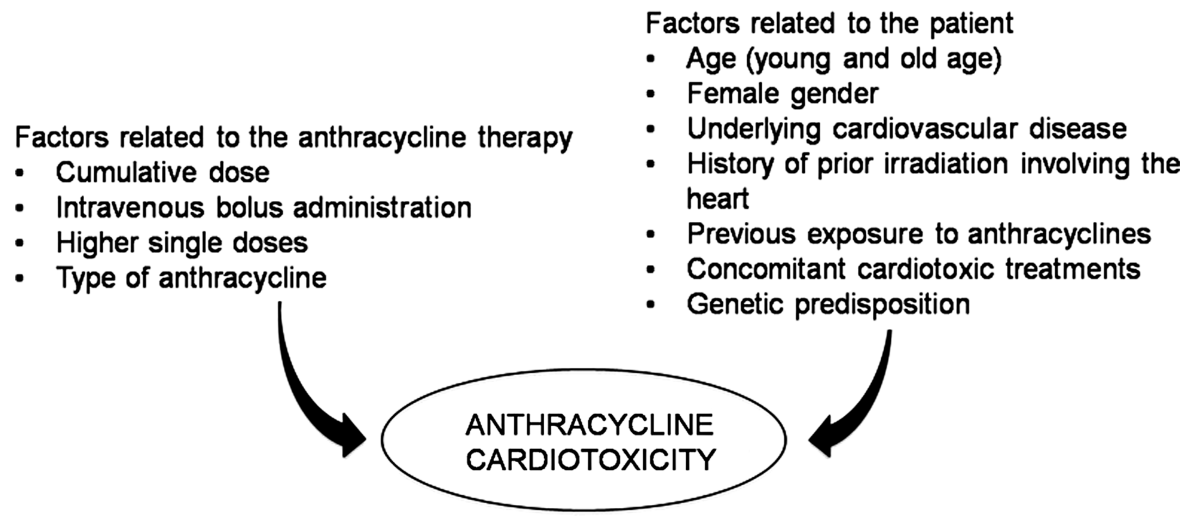

Factors related to the patient

Age (young and old age)

male gender

History of prior irradiation involving the heart

Previous exposure to anthracyclines

Concomitant cardiotoxic treatments

Genetic predisposition
FIG. 2. Simplified algorithm showing the factors related to anthracycline therapy or patient characteristics that may determine cardiac damage. 


\section{A. Adverse effects}

Trastuzumab is a humanized monoclonal antibody that binds the extracellular domain IV of HER/ErbB2 (107, 377). It is the prototypical anti-ErbB2 agent, and the first developed and most widely used type II cardiotoxic drug. Trastuzumab is particularly useful in treating ErbB2 $2^{+}$breast and gastric cancers. Unfortunately, it can also cause CTX in a substantial number of patients, peaking at $28 \%$ with concomitant administration of trastuzumab and ANTs $(262,362,377)$. Indeed, as said earlier, ANTs are responsible for type I CTX, with permanent cardiac damage. Therefore, reduced left ventricle ejection fraction (LVEF) results from the association of trastuzumab and doxorubicin: Trastuzumab enhances or even induces doxorubicin toxicity. Once anti-ErbB2 agents block the protective mechanisms of ErbB2, the oxidative damage induced by doxorubicin increases (91). This co-administration is now avoided. As a class II cardiac dysfunction $(93,94)$, trastuzumab CTX appears to be elicited by the impairment of contractility rather than the loss of cardiomyocytes, and previous chemotherapy seems to be responsible for the troponin release observed in sequential treatment (91).

Pertuzumab is a more recent anti-HER2 antibody that binds the receptor's domain II. Lapatinib is a different anti-ErbB2 agent, a small-molecule inhibitor of the intracellular tyrosine kinase domain of ErbB2. Of note, trastuzumab only disrupts ligand-independent ErbB2 signaling, whereas pertuzumab interferes with the formation of ligand-induced ErbB2 heterodimers. In contrast, lapatinib inhibits both ligand-induced and ligand-independent ErbB2 signaling (69). Interestingly, lapatinib seems to be less toxic than trastuzumab. Data regarding the toxicity of pertuzumab are more limited (262).

\section{B. Mechanisms of adverse effects}

Cardiotoxicity of anti-ErbB2 drugs has been attributed to the inhibition of fundamental actions of neuregulin 1 in the heart $(262,286)$. In brief, in response to various stimuli, in- cluding mechanical strain, adult cardiac microvascular endothelial cells may release neuregulin 1 (NRG1, especially the NRG1 $\beta$ isoform) (221). Thus, NRG1 acts on cardiomyocytes in a paracrine manner, triggering ErbB4/ErbB4 homodimerization and ErbB4/ErbB2 heterodimerization to stimulate protective pathways in response to stress (221, 286). The ErbB2 pathway mediates cell survival and functionality, with recent data focusing on its role in mammalian heart regeneration (63), and appears to be stimulated when the heart experiences adverse hemodynamics or other stress, such as ANT therapies (Fig. 3) (110). It has been postulated that by interfering with the NRG1/ErbB4/ErbB2 axis in the myocardium, anti-ErbB2 agents can cause cardiomyocyte damage and, eventually, HF, and that this is more likely to occur if myocytes are concomitantly exposed to other stressors, such as hypertension or doxorubicin $(70,91)$. Supporting such a hypothesis, ErbB2 cardiac KO mice exhibited dilated cardiomyopathy, with increased susceptibility to ANT-induced damage to cardiac myocytes $(61,291)$. Conversely, overexpression of ErbB2 in the heart resulted in lower levels of ROS in mitochondria, with reduced ROS levels and less cell death in neonatal myocytes isolated from ErbB2(tg) hearts after doxorubicin treatment, due to enhanced levels of glutathione peroxidase 1 (GPx1) protein and GPx activity, with higher levels of two known GPx activators, c-Abl and Arg. These data suggest new mechanisms by which ErbB2 blockers can damage heart structure and function (19).

These data have led to further studies on NRG1/ErbB4/ ErbB2 that have focused away from chemotherapy-induced HF to heart disease from any cause, with implications for new therapeutic perspectives. For example, in mice subjected to pressure overload, ErbB4 and ErbB2 (both mRNA and protein) decreased significantly with the progression of the disease from compensated cardiac hypertrophy to overt $\mathrm{HF}$ (250, 325). Consistently, ErbB2 and ErbB4 receptor expression and activation/phosphorylation were observed to be lower in human-failing myocardia, compared with organ
FIG. 3. ErbB receptor homodimerization or heterodimerization is induced by stressors, including anthracycline therapy, with consequent complex intracellular pathway activation. Cascade effects can be avoided by treatment with monoclonal antibodies and TKIs. See the text for further explanation. EGFR, epidermal growth factor receptor; ErbB2 (or HER2), human epidermal growth factor receptor 2; ERK, extracellular signal-regulated kinase; TKIs, tyrosine kinase inhibitors.

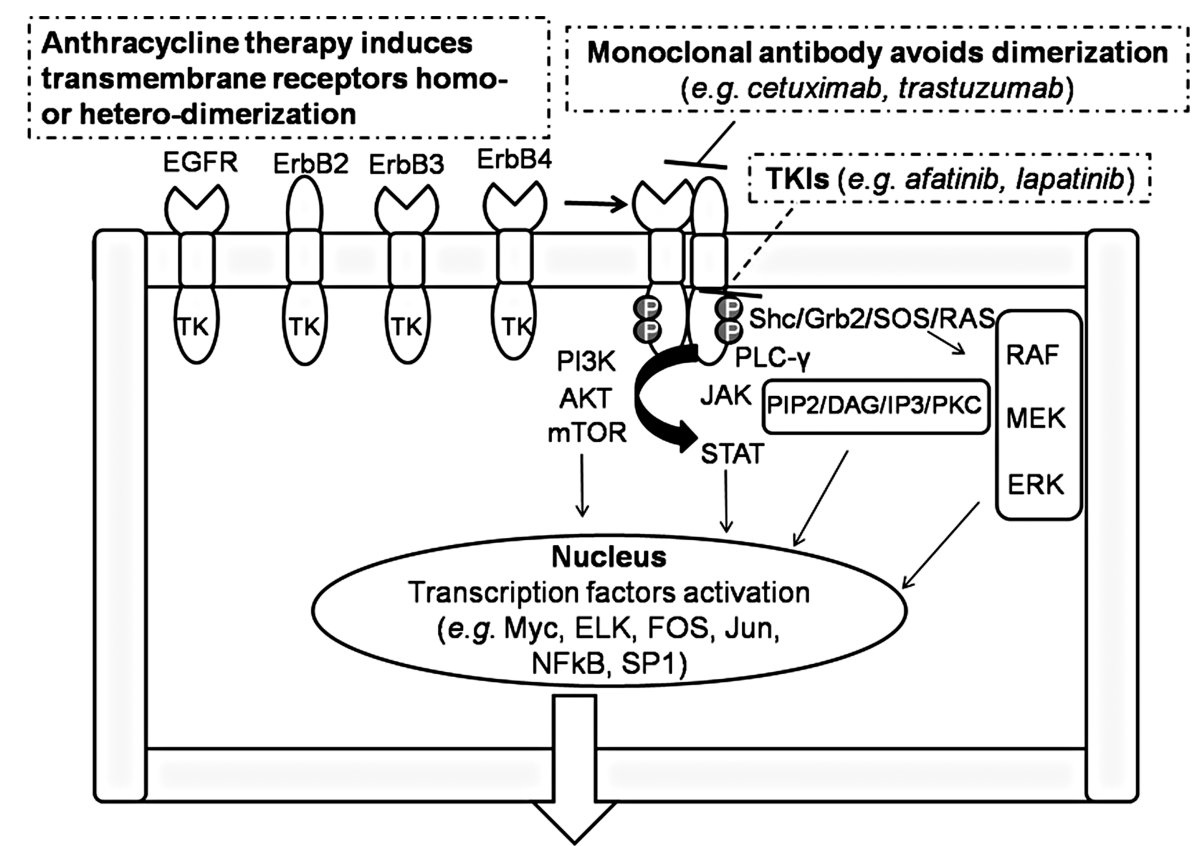

Effects: proliferation, apoptosis, migration, invasion, metastasis, adhesion, remodeling. 
donors (326). Interestingly, LV unloading by implantation of an LV assist device restored the levels of ErbB4 and ErbB2 $(326,400)$. In an apparent contrast with these results, dogs with pacing-induced HF showed increased phosphorylation of ErbB4 and ErbB2 (78). Inactivation of the intracellular downstream effectors of ErbB4 and ErbB2, ERK1/2 and Akt, was observed, suggesting a disabled NRG1/ErbB4/ ErbB2 signaling. Actually, NRG1 expression is increased in HF compared with control conditions in most studies (78, 250, 326). All in all, these data hint that deranged NRG1/ ErbB4/ErbB2 activity is involved in the pathophysiology of $\mathrm{HF}$ in at least two manners: (i) HF may derive from the use of anti-ErbB2 drugs, such as trastuzumab; (ii) ErbB4/ErbB2 is downregulated and/or uncoupled from intracellular signaling despite normal or increased NRG1, possibly leading to cardiac decompensation (250). Furthermore, novel observations suggest that levels of catecholamines, which usually increase with the occurrence of LV dysfunction and with ANT administration $(176,250,282)$, can stimulate ErbB2 expression in myocytes, thus making these cells particularly susceptible to the effects of trastuzumab, resulting in myocardial toxicity (382).

\section{Ways to reduce $\mathrm{CV}$ toxicity}

The aforementioned experimental results may support the use of $\beta$ blockers in the prevention of trastuzumab CTX (see section X.A.1) (281), in line with a retrospective study that found that continuous use of $\beta$ blockers was associated with a lower risk of new HF events in subjects on trastuzumab, ANTs, or both (347). Prevention with $\beta$ blockers is currently being assessed in clinical trials $(180,281,347,349)$ with bisoprolol (MANTICORE 101-Breast) (305), NCT01009918 (carvedilol), and NCT01434134/NCT00806390 (metoprolol) to cure or prevent trastuzumab-induced LV dysfunction $(250,281)$. Interestingly, from the recent PRADA (prevention of cardiac dysfunction during adjuvant breast cancer therapy) trial, we can infer that blocking only $\beta 1$ with metoprolol may not produce an adequate and sufficient cardioprotection (128), thus supporting the use of nonselective $\beta 1$ and $\beta 2$ blockers (382). In the clinical setting of trastuzumab-induced cardiac dysfunction, when trastuzumab is discontinued, normal ErbB2 signaling is restored, and the reduced LVEF can increase back to normal levels. Indeed, trastuzumab re-administration after discontinuation is considered relatively safe on LVEF recovery $(93,94)$.

Animal studies have demonstrated that NRG1 regulates doxorubicin injury in rat myocytes (390). Owing to the aforementioned cardioprotective properties of NRG1 via ErbB4/ErbB2, and as the activity of these receptors is altered in $\mathrm{HF}$, the axis neuregulin-ERB is now being intensively investigated in clinical trials for HF treatment (111, 112, 221). It has been hypothesized that NRG1 and NRG1 analogs can be used as therapeutic agents in HF. Intravenous administration of recombinant human NRG1 and of the glial growth factor 2 isoform of NRG1 $\beta$ enhanced heart function and reduced $\mathrm{LV}$ dimensions in experimental failing hearts $(111,112,218,227,250)$. Since NRG1 exerted positive effects in animal models of ischemia-induced HF even when administered after acute myocardial infarction, it can be speculated that it is able to produce beneficial reverse remodeling of the damaged heart, and it does not simply limit cardiac dilation $(111,112,227)$. Also, it appears that NRG1 exerts an antifibrotic effect, directly inhibiting cardiac fibroblasts and, thus, preventing fibrosis $(111,112)$.

Clinical studies have demonstrated that recombinant human NRG1 is well tolerated by patients, and it ameliorates cardiac dimensions and LVEF until a maximum of 3 months after treatment $(116,169)$. Nevertheless, NRG1 may be considered a growth factor for tumor cells, particularly when administered systemically. Hopefully, additional experimental and clinical studies can assess this fundamental safety concern, producing novel data regarding the effects of NRG1 in $\operatorname{HF}(221,250)$.

\section{VEGF Inhibitors and Multi-Targeted Kinase Inhibitors}

\section{A. Adverse events}

Anti-angiogenic drugs disrupt the VEGF signaling cascade (Fig. 4) and may induce type II CTX (Fig 5). VEGF is a modulator of myocardial function and growth, while, at the same time, modulating the integrity and expansion of the coronary and systemic blood vessels (43, 44, 62, 88, 103, 104, 107, 240, 392). VEGF antagonists may, therefore, produce different forms of CTX, mainly hypertension, thromboembolism (TE), LV dysfunction, and HF (126, 344, 421).

In particular, bevacizumab, sorafenib, and sunitinib are now widely used for the treatment of different cancers. More recently introduced tyrosine kinase inhibitors (TKIs) can also induce CTX. Regorafenib is a multi-target TKI whose targets include VEGF receptor (VEGFR) 1-3, endothelial-specific receptor tyrosine kinase, platelet-derived growth factor

Anti-angiogenic drugs disrupt the VEGF signaling cascade (e.g. bevacizumab, sorafenib, sunitinib, pazopanib and vandetanib)

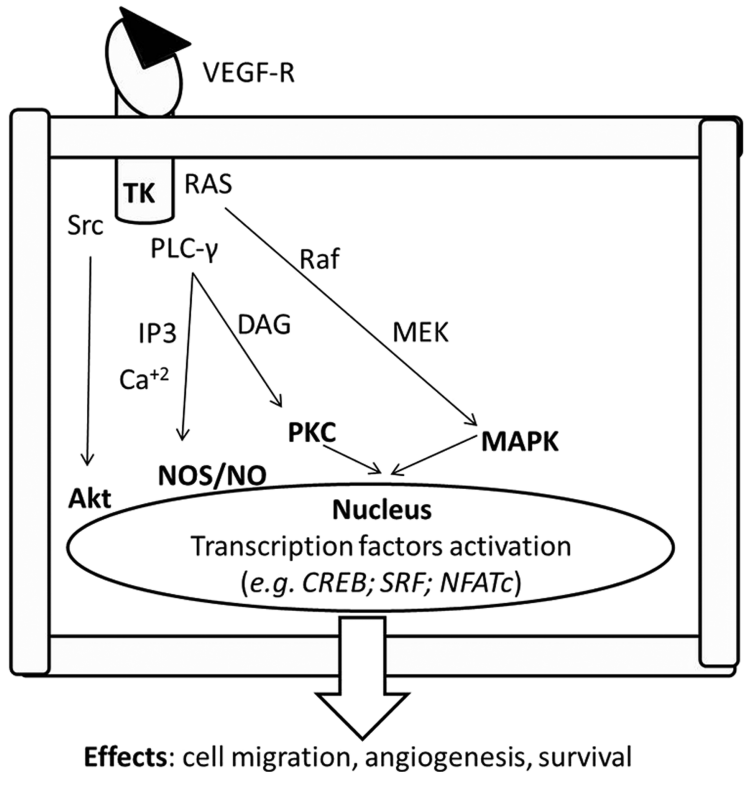

FIG. 4. VEGFR activation triggers a complex intracellular pathway. Cascade effects can be avoided by treatment with anti-angiogenic drugs acting at various levels of the cascade. See the text for further explanation. MAPK, mitogenactivated protein kinase; NO, nitric oxide; NOS, nitric oxide synthase; VEGFR, vascular endothelial growth factor receptor. 
FIG. 5. Schematic illustration of how TKI antagonists induce cardiac damage; these drugs can increase arterial pressure and ventricular-vascular coupling, leading to heart failure. PDGFR, platelet-derived growth factor receptor; VEGF, vascular endothelial growth factor.

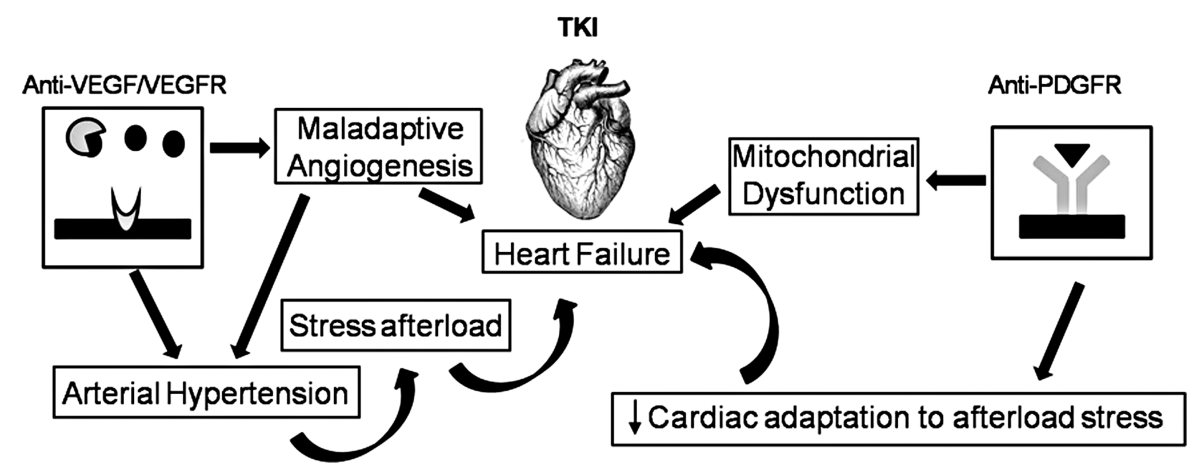

receptor (PDGFR), fibroblast growth factor receptor, cKIT, $\mathrm{RE}$ arranged during transfection (RET), and rapidly accelerated fibrosarcoma kinase. Regorafenib is used in the treatment of colorectal tumors and gastrointestinal tumors (28). It may be responsible for arterial hypertension; less frequently, it can produce cardiac ischemia and myocardial infarction (30).

Pazopanib and axitinib, used in the treatment of metastatic renal tumors, are also associated with a high rate of arterial hypertension. Pazopanib is an orally administered multitargeted TKI, targeting VEGFR 1-3, PDGFA and PDGFB receptors, and c-KIT. In a recent study, the frequency of pazopanib-associated hypertension varied between $36 \%$ and 46\% (268). Axitinib is a third-generation VEGFR inhibitor used in metastatic renal cancer after failure of previous treatments, and it is very selective. In a study comparing axitinib and sorafenib, the frequency of hypertension was $29 \%$ for sorafenib and $40 \%$ for axitinib (162). New anti-angiogenic drugs not yet approved for clinical use are vatalanib and nintedanib. Preliminary evidence indicates a potential risk of arterial hypertension and congestive $\mathrm{HF}$ and, for vatalanib, of pulmonary embolism, although more rarely $(318,407)$.

\section{B. Mechanisms of adverse effects}

The myocardium requires appropriate perfusion to function properly $(43,44,62,88,103,104,107,240,392)$, and it depends on HIF-1 and VEGF pathways, similar to tumors. Of note, inhibition of HIF-1 by p53 may produce HF during chronic pressure overload (336). Moreover, conditional expression of a VEGF scavenger may cause myocardial hibernation and microvessel rarefaction, which can be reversed by suppressing the expression of the scavenger, even months after its induction $(244,417)$. Such findings show that the myocardium is very sensitive to anti-angiogenic treatments, especially with hypertension-related pressure overload.

The antibody bevacizumab binds circulating VEGF-A, which triggers signaling in endothelial cells, and is used as a therapy for advanced lung, breast, and colon/rectum cancers $(160,334)$. It has been observed that bevacizumab can induce cardiac dysfunction in $1 \%$ of chemotherapy-naïve patients and in $3 \%$ of patients with previous chemotherapy (256). Sunitinib and sorafenib are small-molecule TKIs, and they are approved for treating metastatic renal cancer and imatinibresistant gastrointestinal stromal tumors $(45,126)$. Importantly, they are not highly selective, and they can inhibit kinases other than VEGF (43). In particular, sunitinib interferes with $>30$ other tyrosine kinases, including PDGFR alpha and beta, the RET proto-oncogene, FMS-related tyrosine kinase 3 (FLT3), c-Kit, and colony-stimulating factor 1 receptor (CSF1R) $(43,107,135,230)$. This explains why sunitinib is considered more cardiotoxic than other anti-angiogenic drugs, with an incidence of LV dysfunction in a maximum of $28 \%$ of patients $(51,187,269,387)$ (Fig. 5). Indeed, all these kinases play a role in the maintenance of CV function $(9,168$, $217,228)$. The high CTX of sunitinib is also due to interference with off-target kinases, such as ribosomal S6 kinase, with consequent activation of the intrinsic apoptotic pathway, and $5^{\prime}$ AMP-activated protein kinase (AMPK, involved in the response to energy stress), with exacerbation of ATP depletion (107, 186). Interestingly, Hasinoff and Patel (135) not only confirmed the inhibition of AMPK in isolated cardiomyocytes but also suggested that this is likely not essential for the development of sunitinib cardiotoxicity. Creatine kinase may be also involved in the modulation of sunitinib contractile actions (393). A study (51) in mice treated with sunitinib also showed a prolonged opening of the nonselective mitochondrial permeability transition pore (mPTP) and a significant mitochondrial swelling with deformation of the normal mitochondrial architecture in myocytes from hearts subjected to pressure overload. Other studies (422) showed that sunitinib likely does not induce significant impairment of oxidative phosphorylation; thus, the impact of sunitinib on energy metabolism is still controversial, with the incidence of sunitinib-induced myocardial dysfunction being perhaps lower than initially suggested.

In addition, a 2013 manuscript (46) pointed to attention from cardiomyocytes to microvascular dysfunction in the heart and pericyte damage as another important mechanism of cardiotoxicity induced by sunitinib.

Sorafenib appears to inhibit at least 15 kinases, including the VEGFR, PDGFR, Raf-1/B-Raf, FLT3, and c-Kit (43, 107, 392). The incidence of sorafenib-induced CTX is not yet fully characterized. In two meta-analyses that included almost 7000 patients treated with sunitinib and 900 patients treated with sorafenib, rates of $4.1 \%$ for sunitinib-induced HF and $1 \%$ for sorafenib-associated cardiac dysfunction were shown $(77,321)$, but most of these observations were derived from retrospective analyses; only a few trials have assessed LV dysfunction prospectively. The work of Schmidinger et al. (344) showed that 3 out of 14 patients who were administered sorafenib and had cardiac events exhibited abnormal LVEF.

Recently, Feng et al. (98) observed that the axis between the brain-derived neurotrophic factor (BDNF) and a tyrosine kinase receptor (TrkB) is a key player in the regulation of myocardial excitation-contraction coupling, independently and in parallel to $\mathrm{G}$ protein-coupled receptor (GPCR) 
signaling. These data further support the concept that tyrosine kinase blockade with antineoplastic treatments can interfere with fundamental signaling, thus impairing cardiac mechanical work that may produce LV dysfunction (106).

In summary, several mechanisms may lead to cardiac dysfunction in patients treated with anti-VEGF therapy: cKit inhibition, altered activation of MAP with consequent alteration of energy production and mitochondrial function, PDGFR inhibition, and inhibition of signaling favoring cell survival, as well as angiogenesis inhibition and induction of arterial hypertension.

Importantly, hypertension is a main side-effect of all three major anti-VEGF drugs (272). Mechanisms of arterial hypertension include both functional (inactivation of endothelial nitric oxide synthase and production of vasoconstrictors such as endothelin-1) and anatomic (capillary rarefaction) modifications, which lead to vasoconstriction and an increase in peripheral vascular resistance $(272,277,353)$. The main mechanisms of capillary rarefaction are considered the loss of pericytes due to PDGFR inhibition, along with inhibition of angiogenesis by VEGFR inhibition (353). Moreover, renal dysfunction due to angiogenesis inhibition can play a role in inducing and maintaining arterial hypertension. All said, the mechanisms of hypertension induced by antiangiogenic drugs have not yet been fully elucidated, and they are still an unresolved issue.

Bevacizumab produces severe hypertension that, at least in some circumstances, is not reversed by discontinuing the drug. Hypertension from anti-angiogenic agents has been suggested to be a biomarker of anti-cancer drug efficacy, because some patients who developed hypertension were observed to survive longer than those who did not (122). It has been shown that in cases of metastatic colorectal cancer, $20 \%$ of patients showed grade 2-3 hypertension. A partial remission was observed in $75 \%$ of subjects with bevacizumab-induced hypertension, and in only $32 \%$ of those with no hypertension. Moreover, patients who showed grade 2-3 hypertension had significantly longer progressionfree survival than nonhypertensive patients (342).

Sunitinib can also cause hypertension, with an incidence ranging from $5 \%$ to $47 \%$ in different studies. Sunitinib was shown to induce hypertension $(\geq 150 / 100 \mathrm{mmHg})$ in $47 \%$ of the subjects studied, with grade 3 hypertension seen in $17 \%$. High blood pressure was observed within the first 4 weeks of therapy $(32,270)$.

Inhibition of VEGF signaling can also cause arterial and venous thrombosis. Arterial thromboembolic events (ATE) are mainly related to VEGF inhibition and consequent impairment of vascular homeostasis due to a reduction of nitric oxide (NO) synthesis, endothelial dysfunction, and production of vasoactive mediators that favor plaque instability and thrombus formation (212). Bevacizumab causes ATE more frequently in patients contemporaneously treated with other chemotherapeutic agents, in older patients, and in those who have experienced previous thrombotic events (315). The real burden of venous TE related to bevacizumab is less clear (161). The pathogenesis of venous events is always related to impairment of vascular homeostasis due to VEGF inhibition and to production of pro-inflammatory cytokines that favor activation of the coagulation cascade (144).

Sorafenib, pazopanib, and axitinib may also increase the risk of ATE. In a meta-analysis of 10,000 patients, the inci- dence of ATE was $1.7 \%$ in sorafenib-treated patients (49). Venous thrombotic events were reported in $3 \%$ of axitinibtreated patients, with ATE in $2 \%$ and transient ischemic attack in $1 \%$. However, HF was observed in $<1 \%$ of subjects treated with sorafenib and in $3 \%$ of patients treated with pazopanib (324).

Cardiac ischemia or myocardial infarction can also occur with sorafenib $(3.8 \%)$, pazopanib $(2 \%)$, and axitinib $(<1 \%)$ (30). This is mainly related to the inhibition of VEGF signaling, leading to endothelial dysfunction, vasoconstriction, and microvascular rarefaction. Moreover, a reduction in VEGF levels creates an imbalance between cell survival and apoptosis. Endothelial cell apoptosis favors an exposure of sub-endothelial collagen and activation of the coagulation cascade, whereas reduced VEGF levels also interfere with the platelet-mediated fibrinolytic cascade (212).

Finally, patients treated with sorafenib, pazopanib, and vandetanib can present an electrocardiogram (ECG) with QT interval prolongation, which predisposes them to an enhanced risk of ventricular arrhythmias $(194,214)$. In particular, sorafenib can frequently induce QT prolongation $(40.5 \%)$, thus greatly increasing the risk of ventricular arrhythmias (30). Vandetanib has also been related to a moderate risk of QT prolongation (16\%), and more rarely, to life-threatening arrhythmias; in addition, it is associated with an enhanced risk of arterial hypertension (24\%) and HF (21\%) (434). In patients treated with pazopanib, the risk of QT prolongation and torsade de pointes, a dangerous polymorphic ventricular tachycardia leading to sudden cardiac death, is $<2 \%$ (303).

In patients receiving drugs potentially interfering with QT duration, electrocardiographic monitoring of QT interval and periodic control of electrolytes are recommended. A reduction in the drug's dose can be warranted, but rarely discontinuation. Particular attention should be paid to patients with a personal or familial history of QT prolongation or to those who are under concomitant medication that can influence QT interval. For further details on the approaches to reduce CV toxicity, see sections $\mathrm{X}$ and XI.

\section{TKIs and Anti-BCR-abl Agents}

Chronic myeloid leukemia (CML) is a cancer of the white blood cells resulting from the clonal expansion of a transformed multipotent hematopoietic stem cell. It is characterized by a reciprocal translocation between the long arms of two chromosomes, namely chromosomes 9 and 22. This translocation produces a shortened chromosome 22 , the socalled Philadelphia chromosome. The consequence of such translocation is the production of the BCR-ABL1 fusion gene on chromosome 22, and the reciprocal ABL1-BCR gene on chromosome 9. The latter does not have any functional role in CML, whereas the BCR-ABL1 fusion gene is necessary for CML pathogenesis. The ABL1 tyrosine kinase is a master-regulator of the cell cycle regulation, whereas the BCR-ABL1 fusion gene generates a constitutively active tyrosine kinase that leads to uncontrolled proliferation (332).

Targeting the BCR-ABL1 fusion gene has revolutionized the management of CML, turning a fatal disease into a chronic disorder that, in most cases, is compatible with a normal lifespan (339). Based on the data from the IRIS study, which documented a dramatic improvement in survival, 
imatinib was the first targeted drug approved for the therapy of CML (285). However, because of the development of a point mutation in the BCR-ABL1 fusion gene, a considerable number of patients may acquire resistance to imatinib (10). Second-generation TKIs-dasatinib, nilotinib, and bosutinib-have been shown to be effective in imatinibresistant patients, and more rapid in achieving a deep molecular response $(57,182,383)$. Ponatinib, a third-generation TKI specifically designed to overcome resistance caused by a T315I mutation in BCR-ABL1 kinase, has exhibited high clinical efficacy in patients with multi-TKI-resistant CML (56). At their debut in the clinical arena, all five drugs appeared cardiologically safe; however, subsequent information indicated that they may exert adverse CV side-effects that may affect prognosis and quality of life and must be taken into account when selecting treatment agents (267). All these drugs demonstrate significant off-target bindings and off-target effects, which may contribute to both toxicities and therapeutic control of the myeloproliferative disorder $(124,383)$.

Imatinib was originally developed as a PDGFR inhibitor. It is a first-generation TKI that binds to the tyrosine kinase ABL while the protein is in the closed conformation and is inactive. It also inhibits other TKs, including VEGFR members and KIT family kinases (124).

\section{A. Imatinib adverse events}

A 5-year follow-up study on patients with newly diagnosed CML treated with imatinib showed an excellent CV safety record for the drug (285). However, Kerkelä et al. reported clinical data from 10 patients with normal ventricular function who developed severe cardiac dysfunction and New York Heart Association (NYHA) Class 3-4 HF after imatinib treatment, and they demonstrated that mice treated with imatinib develop a severe form of LV contractile dysfunction (185). The publication by Kerkelä et al. prompted many hematologists who had used imatinib to treat multiple patients to review their clinical database; the rates of CTX found were extremely low $(16,113,138,329)$. Further clinical studies then confirmed the excellent $\mathrm{CV}$ risk profile of imatinib $(54,60,64,80,90,148)$.

\section{B. Mechanisms of imatinib-induced adverse events}

Several studies investigated the mechanisms responsible for cardiac toxicity, and they found that imatinib-induced ABL inhibition leads to alterations in the endoplasmic reticulum (ER) stress response by activating the IRE1 kinase arm of the response. IRE1 activates apoptosis signal-regulating kinase 1 and c-Jun N-terminal kinase, which, in turn, causes inhibition of antiapoptotic B cell leukemia/lymphoma and cytochrome $\mathrm{c}$ release from mitochondria, thus leading to mitochondrial function impairment and cell death $(43,44)$. The inhibition of Abl kinase by imatinib is also responsible for PRKR-like endoplasmic reticulum kinase activation, which results in impaired protein import in the mitochondrial matrix, inducing mitochondrial death pathways (409).

Other than the potential proapoptotic effect on cardiomyocytes, imatinib has been shown to exert a number of cardiologically relevant effects. First, it causes fluid retention and edema that may contribute to a mistaken diagnosis of HF. This is probably due to imatinib's inhibition of PDGFR (356). Paradoxically, imatinib treatment can also protect against brain and lung edema during stroke and lung injury, respectively $(39,374)$. It has been demonstrated that in human endothelial cells pretreated with a variety of barrier-disruptive agents, imatinib reduces endothelial barrier dysfunction via inhibition of the abl-related gene $(7,47)$. Imatinib also has favorable effects on glucose control by improving both $\beta$ cell function and peripheral insulin sensitivity $(101,130)$. Imatinib improves hemodynamics and reverses vascular remodeling and cor pulmonale in pulmonary arterial hypertension (PAH) by PDGF inhibition (343). Finally, imatinib was found to attenuate myocardial remodeling and to improve LV diastolic dysfunction in spontaneously hypertensive rats by affecting the PDGFR pathway without the blood pressure-lowering effect (170).

Nilotinib is a structural analog of imatinib that binds and potentially inhibits PDGFR and KIT family kinases, but not kinases of the SRC group (124). It is approved for the therapy of subjects with newly diagnosed $\mathrm{Ph}^{+} \mathrm{CML}$ in any phase. Nilotinib is also approved for CML patients who are resistant to or intolerant of previous therapies, including imatinib.

\section{Nilotinib adverese events}

Early in the development of Nilotinib, there was evidence of QT prolongation, leading to a warning to perform serial ECGs, prevent and correct electrolyte abnormalities, and avoid concomitant drugs potentially responsible for QT interval prolongation (182). However, subsequent trials found no alarming signals of QT-related ventricular arrhythmias (267). Clinical trials, as well as studies analyzing real-life settings, found that nilotinib exacerbates the glycometabolic and lipid profile $(26,166,167,267,313,317)$. Over the past 5 years, several clinical trials, retrospective studies, metaanalyses, and postmarketing surveillance studies have shown that nilotinib-treated patients are at an increased risk of $\mathrm{CV}$ events, especially those related to peripheral artery disease (PAD) $(2,54,64,80,118,148,190,209,267,403)$. Oddly, these vascular adverse events were not reported during the first clinical studies.

\section{Mechanisms of nilotinib-induced adverse events}

Recent studies have documented a number of nilotinibinduced effects on endothelium, platelets, and coagulation, which, together with the metabolic effects, enhance the risk of vascular adverse events $(4,22)$.

As for myocardial toxicity, clinical studies have shown that nilotinib does not cause ventricular dysfunction. This finding concurs with experimental data showing that nilotinib and imatinib produce little or no damage to cardiomyocytes, in comparison with dasatinib, bosutinib, and ponatinib (137).

Dasatinib is a second-generation TKI, and it is more potent than imatinib. Unlike imatinib and nilotinib, dasatinib binds to TKs, in both their active and inactive conformational state. It is classified as a dual Abl/Src inhibitor, although it inhibits a broad spectrum of receptor kinases, including c-Kit and PDGFR (124).

Dasatinib is approved as a frontline therapy of $\mathrm{Ph}^{+} \mathrm{CML}$ patients in the chronic phase, and for salvage treatment of $\mathrm{Ph}^{+}$ CML or $\mathrm{Ph}^{+}$acute lymphoblastic leukemia (ALL) patients who are intolerant of or resistant to other TKIs. 


\section{E. Dasatinib adverse events}

For years, the CV safety profile of dasatinib was considered similar to that of imatinib, with the exception of pleural effusion, which has been observed since the first use of the drug (383). Clinical studies with a long follow-up period found that pleural effusion occurs in one of every three patients, and that half of pleural events occur within the first 2 years of therapy. Pleural effusion requiring drug discontinuation occurs in $<10 \%$ of the cases (351).

$\mathrm{PAH}$ is a rare but serious complication of dasatinib treatment. A transient significant increase of pulmonary arterial pressure detected by echocardiography was observed for the first time in 2017 among patients with pleural effusion (312). Subsequently, other reports emerged of PAH not associated with pleural effusion (263).

In clinical studies, PAH has been observed in $5 \%$ of subjects on dasatinib as a frontline therapy for 5 years and in $2.4 \%$ of patients treated with dasatinib for 7 years, who were resistant to or intolerant of other treatments (351). A recent assessment of all causes of dasatinib-related PAH confirmed by catheterization (41 patients) showed that a unique feature of dasatinib-induced PAH is different complete or partial reversibility on drug discontinuation (352). However, the French PH Registry that identified nine cases of dasatinibassociated PAH reported that most patients did not experience complete recovery, and it recorded two cases of cardiac death after dasatinib withdrawal (263).

Analysis of the FDA database designed to support postmarketing surveillance found that PAH was associated with dasatinib and not with other TKIs, and that it often occurred in patients with $\mathrm{CV}$ risk factors or a medical history of cardiopulmonary events (54).

\section{F. Mechanisms of dasatinib-induced adverse events}

Pleural effusion is considered the result of PDGFR inhibition, particularly of the PDGFR- $\beta$ isoform, of which dasatinib is the most potent inhibitor; however, other mechanisms have been suggested (184). Due to the high frequency of lymphocytes in pleural fluid and tissue and the association with skin rash or history of autoimmunity, an autoimunne mechanism has been hypothesized (297). It has been suggested that an immunemediated mechanism underlying the occurrence of pleural effusion could help to promote tumor regression. Patients who develop lymphocytosis and increased levels of specific lymphocyte subsets in association with pleural effusion have a higher major molecular response rate and better progressionfree and overall survival than patients who do not (89).

The underlining molecular mechanism responsible for PAH is still poorly understood. Since dasatinib is a potent pan-Src inhibitor, it has been suggested that Src inhibition is pivotal in the development of PAH (264). The evidence that CML patients treated with dasatinib may display high circulating levels of E-selectin, intercellular adhesion molecule, and vascular cell adhesion molecule, and the observation that cultured pulmonary endothelial cells administered with dasatinib display an increased mitochondrial ROS production and apoptosis support the notion that dasatinib-induced PAH is caused by endothelial dysfunction (127).

Because vascular events have been described in patients treated with other TKIs, recent clinical trials have evaluated the incidence of coronary, cerebral, and peripheral throm- botic events in dasatinib-treated patients; a slightly higher risk was found, compared with imatinib. However, a recent meta-analysis showed that the use of dasatinib is associated with a significant increase in the risk of CV events. In a population-based cohort study, the incidence of myocardial infarction was 2.4 times higher in patients treated with dasatinib than in those treated with imatinib.

Bosutinib is a second-generation, dual Src/Abl TKI lacking significant PDGFR or c-KIT binding properties (124). Bosutinib is currently approved only for patients with $\mathrm{Ph} 1$ chronic-phase CML who were resistant to or intolerant of previous TKI therapy.

\section{G. Bosutinib adverse events}

Studies that evaluated bosutinib enrolled patients newly diagnosed with CML, or patients resistant to or intolerant of other TKIs; these studies documented a low incidence of cardiac and vascular events $(31,55,57,58)$. Similar to other TKIs, the incidence of adverse events was higher in secondor later-line treatment compared with first-line treatment. $\mathrm{HF}$ and coronary or PAD-related events were rare, suggesting that the cardiotoxic profile of bosutinib differs from that of other second- or third-generation TKIs. Most events occurred within the first year of therapy, and very few patients discontinued treatment because of these events, which were mostly managed with concomitant medications (55).

It should be noted that in a first-line setting, the overall incidence of events and dose reduction or interruption is similar for imatinib and bosutinib, even though pericardial disorders occurred more often in bosutinib-treated patients than in those treated with imatinib.

Ponatinib, a third-generation BCR-ABL TKI, was originally developed for the inhibition of pan-resistant $A B L$ $\mathrm{TK}$, and it is clinically effective in imatinib-, nilotinib-, and dasatinib-resistant CML. Ponatinib targets BCR-ABL in its inactive conformation and inhibits T315I and other BCRABL mutants. It also targets a broad spectrum of TK receptors, including epidermal growth factor receptor (EGFR), PDGFR, VEGFR, SRC kinase, c-KIT, FLT3, and RET (124).

\section{H. Ponatinib adverse events}

Ponatinib was tested in $\mathrm{Ph}^{+}$patients with refractory CML or ALL enrolled in phase I (65 patients) and in phase II (449 patients) of the PACE trial. Both studies documented a high efficacy of the drug, accompanied by an increased risk of arterial thrombotic events $(56,59)$. For these reasons, ponatinib was approved with a warning of the risk of serious vascular events. Findings from these studies provided the rationale to perform a phase III study (EPIC) to test the hypothesis that ponatinib may lead to a deep molecular response in patients newly diagnosed with CML, and to assess its safety in patients with no previous exposure to other TKIs (226). The extended follow-up periods of the phase I and II studies documented that cardiac, cerebral, and peripheral thrombotic events continued to occur; this finding determined the early termination of the phase III study and led ARIAD Pharmaceuticals and the FDA to withdraw ponatinib from the market in October 2013. In January 2014, after a reassessment of the benefit/risk ratio of ponatinib, the FDA allowed its reintroduction into the market, with a recommendation to take appropriate measures to mitigate adverse effects in 
patients. The mechanisms of ponatinib $\mathrm{CV}$ toxicity are still poorly understood. Diagnostic and pharmacological measures aimed at reducing the $\mathrm{CV}$ risk were not included in the protocols of the phase I-III trials. The implementation of these preventive measures may help to improve the therapeutic index of ponatinib in the clinical setting (27). For further details on the approaches to reduce TKIs-induced CTX, please refer to sections X and XI.

\section{Taxanes}

\section{A. Adverse effects}

Although the potential of taxanes to induce cardiac dysfunction was likely overestimated in the past, they deserve a brief note. The most commonly used taxanes are docetaxel and paclitaxel, which are still used in breast, nonsmall cell lung, and ovarian cancers. Among the adverse effects of these drugs, a high incidence of arrhythmias has been observed, including asymptomatic sinus bradycardia (330), which usually is not a major problem for patients. A large range of conduction blocks and cardiac ischemia have been also observed in a few patients participating in trials (330). Ventricular arrhythmias are far less common: Only $0.26 \%$ of patients experienced ventricular tachycardia and ventricular fibrillation, and many of them were also co-treated with cisplatin $(245,330)$. In a clinical trial (EORTC 24971/TAX 323 Study Group), patients treated with docetaxel displayed a $1.7 \%$ incidence of cardiac ischemia (411). In about 3500 patients treated with paclitaxel followed by the Cancer Therapy Evaluation Program's Adverse Drug Reaction database, the overall incidence of severe (grade 4 and 5) cardiac events was $<0.3 \%$ (12). These severe events were observed till 2 weeks after the beginning of paclitaxel treatment. Of note, many of these subjects had important $\mathrm{CV}$ risk factors, including coronary disease and hypertension.

\section{B. Mechanisms of adverse effects}

Taxanes exert anticancer effects, acting as anti-microtubule agents and fostering polymerization of tubulin. In this way, these agents induce microtubule dysfunction and alter cell division. Moreover, they may affect histamine release (330). These taxane-induced effects may perturb cardiac function. Brady-arrhythmias may be due to stimulation of histamine $\mathrm{H}_{1}$ and $\mathrm{H}_{2}$ receptors. The latter receptors may increase the possibility of reentry of depolarization, which is at the basis of ventricular ectopy $(245,330)$. Ischemia and increased myocardial oxygen demands render the heart more susceptible to arrhythmias. Of note, paclitaxel in combination with ANTs increases the incidence of CTX, which appears at lower cumulative doses with respect to ANTs alone (120). In particular, plasma levels of doxorubicin and the formation of the toxic metabolite doxorubicinol in myocytes can be enhanced by taxanes.

\section{Ways to reduce $\mathrm{CV}$ toxicity}

Cardiotoxic symptoms are frequently resolved on discontinuation of taxanes. Since histamine is considered a relevant player in taxane CTX, treatment with corticosteroids and antihistamines may reduce the incidence of clinically significant arrhythmias $(245,330)$.

\section{Cancer Immunotherapy}

All components of the innate and adaptive immune system are important in the recognition and elimination of tumor cells (immune surveillance) (133). These immune components recognize specific neo-antigens that are expressed by tumor cells. However, the production of immunosuppressive cytokines/chemokines, the presence of immunosuppressive cells, or the expression of inhibitory checkpoints within the tumor microenvironment allow the tumor cells to escape immune surveillance (296).

\section{A. Immune checkpoint inhibitors}

Tumors exploit immune checkpoints to evade antitumor immunity. The success of cancer immunotherapy over the past decades has revolutionized the management of a wide array of malignancies. At the forefront of immunotherapy are immune checkpoint blockers, which have seen unprecedented success in cancer treatments because of their broad bioactivity across many tumor types. The two most prominent to date are the targeting of cytotoxic $\mathrm{T}$ lymphocyte-associated protein 4 (CTLA-4) and the interaction between the proteins programmed cell death 1 (PD-1) and programmed death-ligand 1 (PD-L1).

\section{B. $C T L A-4$}

It has structural homology to the co-stimulatory molecule CD28, and it can also bind B7 molecules on antigenpresenting cells (APCs) with a higher affinity than CD28. CTLA-4 is a competitive antagonist of the CD28-B7 interaction in that it blocks co-stimulation at the T cell-APC interaction, thus suppressing activation of its cell response (200) (Fig. 6). Ipilimumab and tremelimumab are monoclonal antibodies for clinical use against CTLA-4 (117, 354, $355,431)$.

\section{PD-1/PD-L1 pathway}

PD-1 is a checkpoint receptor that, by binding with its ligands (either PD-L1 or PD-L2), inhibits T effector functions by dampening signaling downstream of the $\mathrm{T}$ cell receptor (TCR) (397). Therefore, the expression of PD-L1 in the tumor micro-environment protects cancer cells from immunemediated destruction. Several monoclonal antibodies against the PD-1/PD-L1 axis have been developed and/or approved as anti-cancer agents $(396,397)$.

PD-1, similar to CTLA-4, is expressed in activated cells, and its activation downregulates signaling that is mediated on antigen recognition by the TCR (354). PD-1 has two ligands, PD-L1 and PD-L2. PD-L1 can be expressed in many cell types, including tumor cells, immune cells, epithelial cells, and endothelial cells (354). PD-L2 is predominantly expressed in APCs. Pembrolizumab and nivolumab are PD-1-blocking monoclonal antibodies that are approved for treating different types of cancers. Atezolizumab is a PD-L1 targeted monoclonal antibody recently approved for treatment of certain tumors (354). Other inhibitory molecules (e.g., LAG3, OX40, TIM3, TIGIT) are being assessed for their potential utility as immunotherapeutic targets $(15,211,279,399)$. 

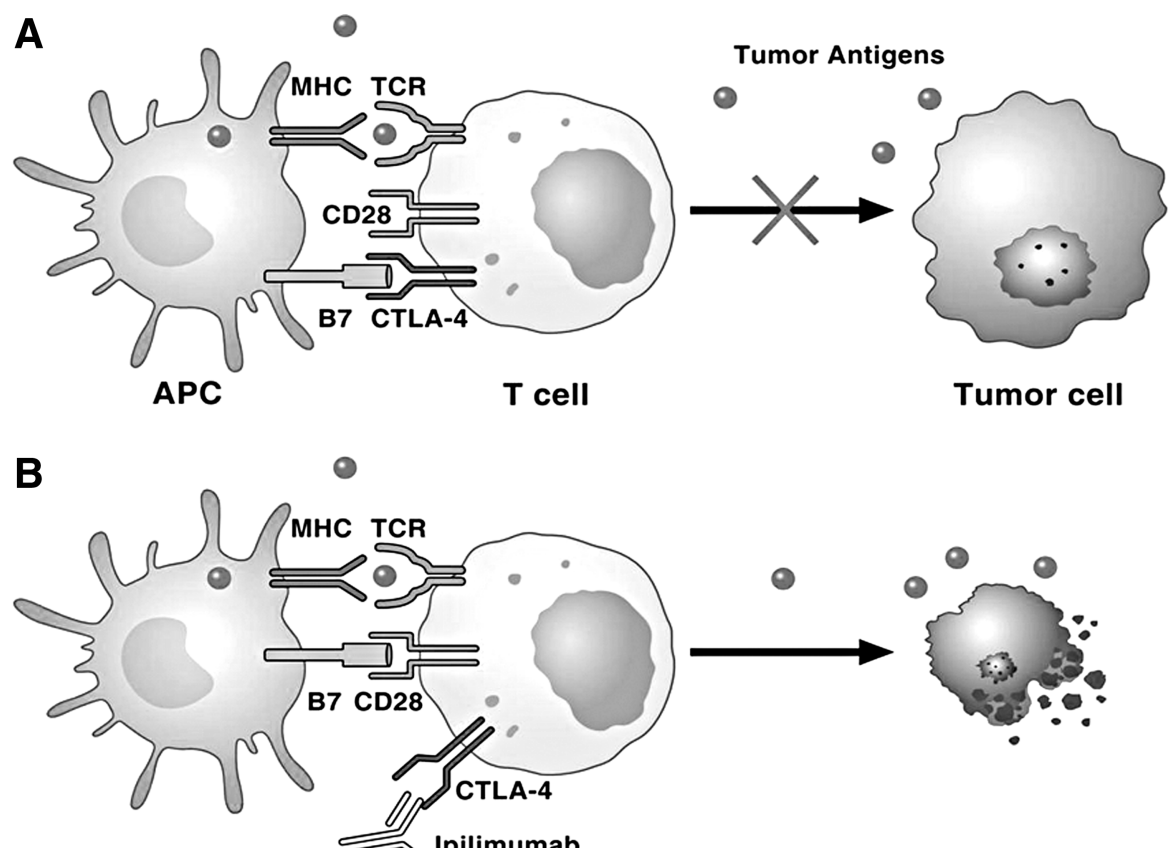

FIG. 6. Role of CTLA-4 and its interaction with ipilimumab in tumor immunity. (A) Dying tumor cells release tumor neo-antigens that are taken up by APCs, which then present B7 co-stimulatory molecules to $\mathrm{T}$ cells. $\mathrm{T}$ cells recognize tumor neo-antigens on APCs and are activated. Activated $\mathrm{T}$ cells upregulate inhibitory checkpoints, such as CTLA-4 and PD-1, which block $T$ cell activation and attack of tumor cells. (B) Ipilimumab blocks CTLA-4, resulting in $\mathrm{T}$ cell activation and destruction of tumor cells. See the text for further explanation and acronyms. APCs, antigen-presenting cells; CTLA-4, cytotoxic T lymphocyteassociated protein 4; PD-1, programmed cell death $1 ; \mathrm{TCR}, \mathrm{T}$ cell receptor.
APC

$T$ cell

\section{Combination of checkpoint inhibitors}

CTLA-4 or PD-1/PD-L1 blockade as a monotherapy has shown impressive tumor regression in some patients with different solid and hematological cancers $(95,225,241)$; however, the overall long-term survival rate should be improved (304). Ongoing clinical trials combine different checkpoint inhibitors. In addition, some clinical trials combine checkpoint inhibitors with angiogenesis inhibitors (i.e., bevacizumab), EGFR, TKIs or BRAF inhibitors, and chemotherapy or radiation therapy (396).

\section{E. Checkpoint inhibitors and immune-related adverse events}

Checkpoint inhibitors can cause a novel spectrum of adverse events called immune-related adverse events (IRAEs) (254, 420 ), because they can induce autoimmune manifestations ( 75 , 202). The most common IRAEs after treatment with CTLA-4 inhibitors include rash, diarrhea, colitis, hepatotoxicity, and endocrinopathies $(115,149)$. Immune-mediated CTX produced by these compounds has generally been observed in individual cases with variable presentations, comprising pericarditis and myocarditis $(117,141,414,431)$. The largest case series to date describing CTX after treatment with checkpoint inhibitors reported seven cases (141).

Monoclonal antibodies targeting PD-1 (such as pembrolizumab, nivolumab, and pidilizumab) or PD-L1 (atezolizumab, durvalumab) have shown a favorable toxicity profile in several trials (82). However, occasional reports of myocarditis after nivolumab (151) or pembrolizumab (207) treatment have been reported.

Although so far, there have been few case reports on cardiac adverse effects in patients treated with checkpoint inhibitors, several notes of caution should be considered. First, experimental data in PD-1-deficient mice are associated with dilated cardiomyopathy and sudden death by congestive

\section{Killing of Tumor cell}

HF $(280,288)$. In addition, PD-1 protects against inflammation in T cell-mediated myocarditis (385). Finally, PD-1 deficiency leads to the development of fatal myocarditis in mice genetically predisposed to autoimmunity (418). The latter observation is particularly important, because patients with underlying autoimmune disorders have always been excluded from clinical trials with checkpoint inhibitors. Therefore, the real-life risk of cardiac adverse events in patients with autoimmune disorders treated with checkpoint inhibitors is so far unknown.

Checkpoint inhibitors have only recently been introduced, and there are limited data on late-onset IRAEs after several years of initiating treatment. Careful follow-up of cardiac adverse events in long-term treated patients is warranted. Finally, and importantly, treatment with checkpoint inhibitors or sequential therapy with different checkpoint inhibitors, in association with chemotherapy or radiation therapy, is on the rise. Therefore, a combination or sequential therapies may contribute to some of the adverse cardiac effects caused by chemotherapy or radiation therapy.

\section{Antimetabolites}

Fluoropyrimidines, such as 5-fluorouracil (5-FU), capecitabine, and gemcitabine, are used in the therapies of solid tumors such as gastrointestinal, breast, head, neck, and pancreatic cancer. 5-FU reaches higher levels in cancer cells than in systemic circulation when administered intravenously. One 5-FU pro-drug is capecitabine, which is converted into its active form after oral administration $(11,188,191,253$, 278, 302).

\section{A. Adverse effects}

Although well tolerated, both capecitabine and its main compound can induce CTX. The onset of capecitabine CTX is generally rapid. It usually occurs within 4 days from the 
start of the first cycle of chemotherapy (174, 175). Prospective and retrospective studies have shown that previous events of cardiac ischemia are the strongest risk factors for fluoropyrimidine-induced CTX (195, 309, 310). ECG abnormalities have been found in patients treated with a 24-h 5-FU infusion in combination with capecitabine. Palpitations and chest pain are the most common clinical manifestations, accompanied by new-onset ECG alterations, such as ST segment deviation, sinus tachycardia, QT prolongation, and signs of myocardial ischemia and acute coronary syndromes. Less frequent are arterial hypertension, ventricular arrhythmias, LV dysfunction, HF, and cardiogenic shock. Cardiac arrest and sudden death are very rare events, and they account for $<1 \%$ of clinical manifestations $(5,198,309,408)$.

The incidence of CTX produced by 5-FU varies between $0 \%$ and $35 \%$, with a mortality rate ranging between $2.2 \%$ and $13.3 \%$, depending on the dose, cardiac comorbidities, and route of administration (198, 309, 310). Larger trials and meta-analyses on breast and colorectal cancer patients, however, have reported an incidence of symptomatic CTX of $1.2 \%-4.3 \%$ during treatment $(205,407)$. A recent study found silent ischemia due to the cardiac stress test in 6\%$7 \%$ of 5-FU-treated patients (216). Unfortunately, patients without overt $\mathrm{CV}$ risk factors are often treated as outpatients. As a result, symptomatic and asymptomatic cardiac events remain undiagnosed because of inadequate cardiac monitoring. This can lead to a discrepancy between observed and actual CTX, in terms of both symptomatic and asymptomatic patients.

\section{B. Mechanism of adverse effects}

Although 5-FU has a brief half-life, active metabolites are retained in all tissues, including cardiac and cancer tissues, resulting in a prolonged exposure of cells to the drug (198, $215,261)$. Thymidine phosphorylase, the pivotal enzyme involved in the conversion of capecitabine to 5-FU, is highly expressed in both atherosclerotic plaques and cancer tissues, explaining the higher prevalence of capecitabine-induced CTX in subjects with coronary artery disease (CAD). Nevetheless, the pathophysiological mechanisms underlying CTX from antimetabolite drugs remain poorly understood. Several mechanisms have been proposed (Fig. 7): (i) Reduction in synthesis of NO, with consequent coronary spasms and endothelium-independent vasoconstriction via the protein kinase pathway $(52,296,357)$; (ii) increased intracellular ROS/RNS, with consequent oxidative stress and cardiomyocyte apoptosis (205); (iii) increased endothelial thrombogenicity, with consequent induction of acute coronary syndromes (180); and (iv) interference with DNA and RNA growth by substituting for the normal building blocks of RNA and DNA.

The existence of a synergy between capecitabine and other anti-cancer drugs has also been suggested. Several studies have shown that CTX is more frequent in subjects administered a combined therapy of capecitabine and either taxanes or lapatinib than in patients treated with capecitabine alone $(105,174,175,278)$.

Studies in rabbits have shown that a single high dose of capecitabine caused hemorrhagic infarction of the ventricle walls, proximal spasms of the coronary arteries, and death
Mechanism of Antimetabolites Cardiotoxicity (e.g. 5-FU, capecitabine and gemcitabine)

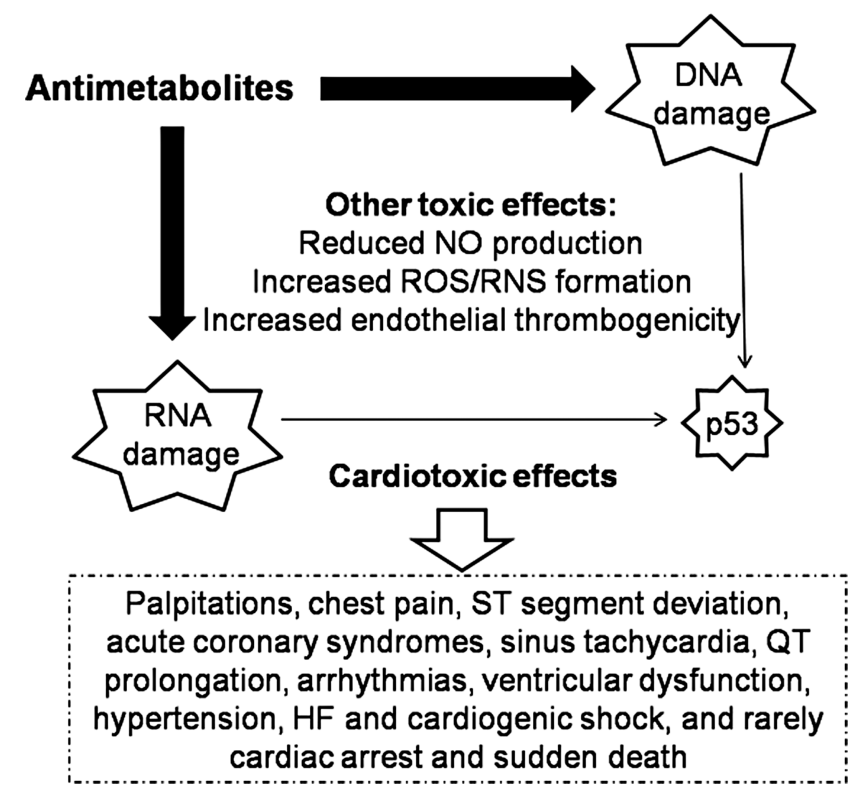

FIG. 7. Main cardiotoxic mechanism and effects induced by antimetabolite drugs. See the text for further explanation. 5-FU, 5-fluorouracil; HF, heart failure; RNS, reactive nitrogen species; ROS, reactive oxygen species.

within a few hours from intravenous injection. In contrast, repeated lower doses produced LV hypertrophy, concentric fibrous thickening of the coronary intima, and foci of necrotic myocardial cells (398). Due to the importance of redox stress in antimetabolite-induced CTX, please refer to sections X and XI for ways to reduce this toxicity.

\section{Proteasome Inhibitors}

Proteasome inhibitors are relatively novel anti-cancer therapies, which are particularly useful in the therapy of multiple myeloma and other hematologic conditions (amyloidosis, non-Hodgkin lymphoma). The first proteasome inhibitor approved for the treatment of cancer was bortezomib; second-generation drugs include carfilzomib, ixazomib, delanzomib, oprozomib, and marizomib (79, 201, 276).

\section{A. Adverse effects}

Experimental data suggest that chronic (over 12 weeks) proteasome inhibition is associated with systolic dysfunction and increased atherosclerosis $(143,284)$. A meta-analysis conducted by Xiao et al. reported that the incidence of CTX in subjects treated with bortezomib varied with tumor type and treatment regimen, in the range of $2.3 \%-3.8 \%$, with a mortality rate of $3.0 \%$ (427).

Treatment with proteasome inhibitors may be associated with hypertension, HF, myocardial infarction, and cardiac arrest, especially if such therapies are associated with concomitant use of doxorubicin, lenalidomide, and steroids, or in patients with a history of cardiac events $(17,136,151$, $373,423,424)$. The incidence of $\mathrm{HF}$ in subjects receiving bortezomib is relatively low (up to 4\%) compared with 
carfilzomib (up to 25\%), a more potent and irreversible proteasomal inhibitor; patients treated with higher doses $\left(\geq 36 \mathrm{mg} / \mathrm{m}^{2}\right)$ of carfilzomib have a higher risk of cardiac toxicity $(65,328,331)$.

\section{B. Mechanisms of adverse effects}

Proteasome inhibitors are compounds that block the activity of proteasomes, protein complexes that play a key role in degrading dysfunctional or unneeded proteins; these cellular complexes that break down proteins are particularly important for the functional maintenance of cardiomyocytes. Therefore, cardiac dysfunction may be expected if the function of these complexes is impaired.

Many mechanisms of chemotherapy-induced cardiotoxicity still remain to be clarified. It has been suggested (284) that bortezomib alters the function of cardiomyocytes through the impairment of mitochondrial energetics. The cardiomyocytes are contractile cells with a very high demand for ATP and may be particularly sensitive to agents that disrupt mitochondrial activity, such as the proteasome inhibitor bortezomib. Furthermore, a reduced synthesis of ATP could trigger the capillary tunneling, as revealed by a histopathological examination of heart sections of rats treated with bortezomib (284). Chronic uptake inhibitor of the proteasome is associated with increased oxidative stress at the level of the intima of the epicardial coronary arteries, resulting in thickening of the vessel wall, which can trigger premature atherosclerosis (143).

The addition of other chemotherapeutic agents such as ANT with proteasome inhibitors improves the effectiveness of antineoplastic therapy; however, this combination of drugs can cause cardiotoxicity. Spur et al. (368) have analyzed the function of the proteasome in primary cardiomyocytes treated with doxorubicin in the presence of proteasome inhibitors. Interestingly, the authors concluded that, contrary to carfilzomib, which targets both the $\beta 5$ standard proteasome and the LMP7 immunoproteasome subunit, immunoproteasomespecific inhibitors with known anti-tumor capabilities for specific cancer cells, such as multiple myeloma, may be advantageous to reduce the mortality of cardiomyocytes, when there is a combination therapy, and, therefore, may be envisioned as a way to reduce CV toxicity, when compared with traditional proteasome inhibitors.

\section{Ways to reduce CV toxicity}

The initial step in $\mathrm{CV}$ management of subjects treated with proteasome inhibitors is to assess their baseline risk for CTX, by taking a clinical history and conducting an examination. More frequent surveillance may be warranted for subjects with higher baseline clinical risk or abnormal cardiac baseline function.

CTX induced by proteasome inhibitors may be reversible in some patients with prompt cessation of these therapies and initiation of traditional HF treatments (123). ACE-Is or angiotensin II receptor blockers (ARBs) in combination with $\beta$ blockers are recommended in patients with symptomatic $\mathrm{HF}$, similar to the general HF population and in asymptomatic cardiac dysfunction, to prevent further cardiac dysfunction or the development of symptomatic HF in patients at a high risk (432).
Single-center experiences have shown a decrease in the occurrence of cardiac events when infusion time of carfilzomib was settled to $30 \mathrm{~min}$ and a cardioprotective activity of dexrazoxane was exhibited $(17,136)$.

In some cases, re-administration of carfilzomib with dose modification is possible. Long-term surveillance should be considered for patients who developed CTX during these therapies and for those in whom cardioprotective treatments have been started, to confirm recovery or to detect irreversible cardiac dysfunction.

\section{The Importance of Mechanisms Such as Oxidative and Nitrosative Stress in CV Toxicity: An Overview}

As reported earlier, the cardiotoxic mechanisms of several anticancer agents involve an unbalanced generation of ROS and RNS, leading to the so-called oxidative/nitrosative stress. ROS/RNS imbalance may derive from increased production or the inactivation of endogenous antioxidant enzymes by antineoplastic drugs, overwhelming the body's defenses. Moreover, antioxidant resources (especially catalase) are lower in the cardiac tissue compared with other organs (e.g., the liver), making the heart more vulnerable to ROS/RNS injury $(257,258)$.

Low physiological levels of ROS/RNS regulate many CV physiological functions (Fig. 8). For instance, $\mathrm{H}_{2} \mathrm{O}_{2}$ produced by mitochondria is considered an endothelium-derived hyperpolarizing factor and a metabolic vasodilator of the coronary circulation (294).

High levels of ROS/RNS, overwhelming cellular antioxidant defense systems, may damage biomolecules and dysregulate cellular signaling pathways in the CV system. In particular, ROS/RNS can lead to membrane lipid peroxidation with subsequent membrane damage. ROS/RNS can also lead to DNA damage, and it may trigger apoptotic cell death (Fig. 9). Cascade effects would include vacuolization and cardiomyocyte replacement by fibrous tissue, leading to irreversible damage $(204,219,248-250,333,353,376,434)$.

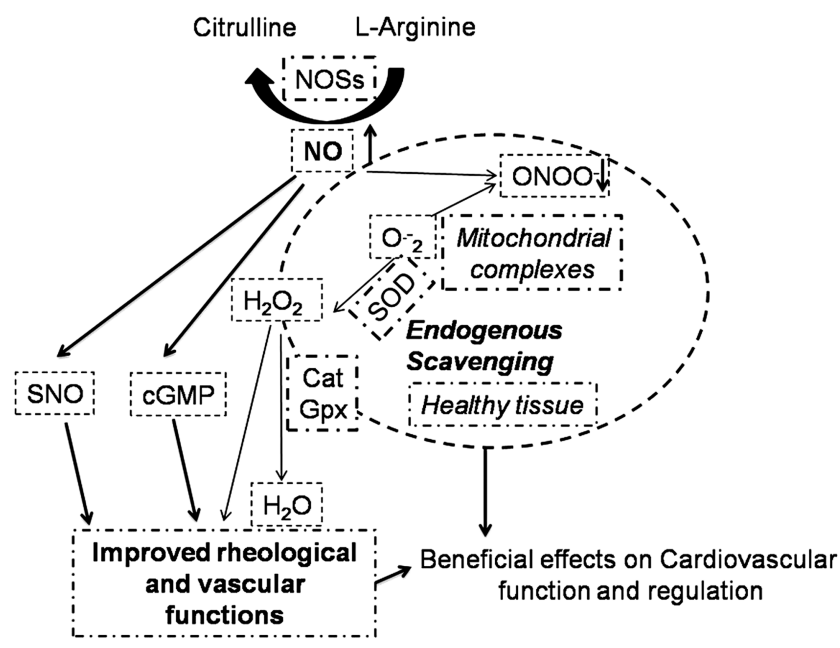

FIG. 8. Physiological function of the redox system. See the text for further explanation. GPx, glutathione peroxidase; $\mathrm{H}_{2} \mathrm{O}_{2}$, hydrogen peroxide; NOSs, nitric oxide synthases; ONOO- ${ }^{-}$, peroxynitrite; SNO, S-nitrosylation; SOD, superoxide dismutase. 


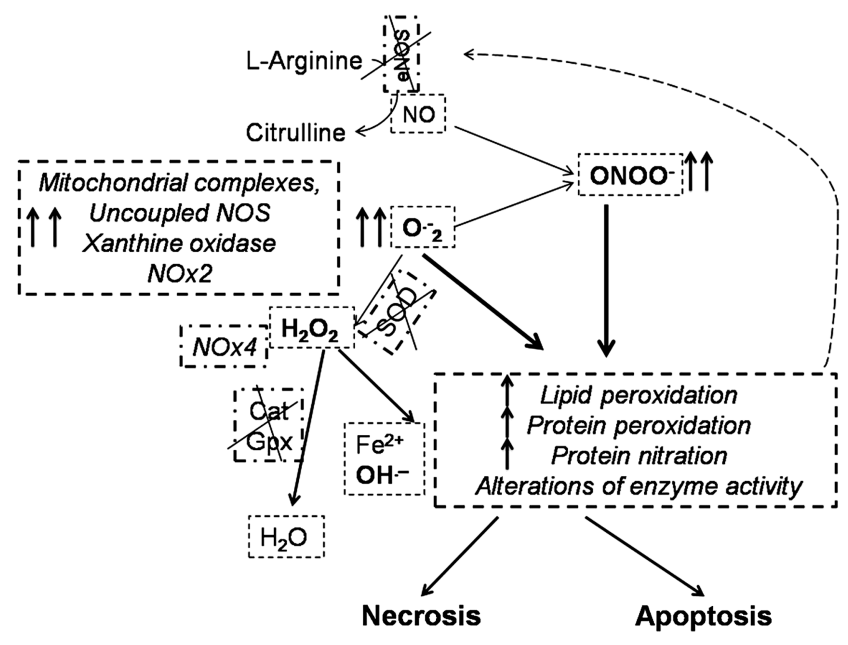

FIG. 9. In the case of excessive production of superoxide anion $\left(\mathrm{O}_{2}{ }^{--}\right)$and other ROS/RNS, lipid and protein oxidation/nitration may occur, with consequent alteration of the activity of many enzymes, leading to cell death. The dashed arrow represents the onset of a vicious cycle affecting redox enzymes. See the text for further explanation. NOX, NAD(P)H oxidase.

In the CV system, ROS are produced by many mechanisms and enzymes. Among the main ROS producers, there are xanthine oxidases and $N A D(P) H$ oxidases (NOXs), as well as uncoupled nitric oxide synthases (NOSs), peroxisomes, and mitochondrial complexes; ROS production may vary considerably among these enzymes $(294,323,394)$. All these ROS producers are affected by multiple anti-cancer drugs (412).

The heart is rich in mitochondria, which occupy $36 \%-40 \%$ of the myocyte volume and produce $\sim 90 \%$ of the cellular energy. Hence, derangements of mitochondrial function could be particularly critical in cardiomyocytes (293, 294, 394). Cardiomyocyte complexes of the mitochondrial respiratory chain, especially complexes I and III, are the main source of superoxide anions $\left(\mathrm{O}_{2}{ }^{\bullet-}\right)$. A common mechanism of cell death (necrosis and/or apoptosis) involves the opening of the nonselective mPTP, which is located between the mitochondrial membranes. The opening of mPTPs is facilitated by high ROS levels in the presence of $\mathrm{Ca}^{2+}$ overload and cyclophilin $\mathrm{D}$ activation. This pore opening allows the free passage of low-molecular-weight solutes $(<1.5 \mathrm{kD})$, including cytochrome $\mathrm{c}$, into the cytosol, where they can activate the apoptotic process. Moreover, a vicious cycle of ROSinduced ROS release takes place, leading to massive ROS production. If the mPTPs remain open, the mitochondria swell and expand owing to osmotic pressure, and ROS undermine membrane structure via lipid peroxidation; thus, these processes disrupt the mitochondrial membrane and favor mitochondrial rupture and cell death $(293,294)$.

Another ROS source strongly affected by antiblastic agents is represented by the $N A D(P) H$ oxidase system, which comprises several isoforms, namely NOXs 1-5 and dual oxidases DUOX1/2. Myocardial NOX2, usually quiescent, produces $\mathrm{O}_{2}{ }^{\bullet-}$ when activated; whereas $\mathrm{NOX} 4$, which is constitutively active, generates hydrogen peroxide $\left(\mathrm{H}_{2} \mathrm{O}_{2}\right)$. Moreover, superoxide dismutases (SODs) may convert $\mathrm{O}_{2}{ }^{\bullet-}$ to $\mathrm{H}_{2} \mathrm{O}_{2}$, which is a relatively stable molecule. In specific compartments, including mitochondria, $\mathrm{H}_{2} \mathrm{O}_{2}$ may be con- verted to oxygen and water by catalase and by glutathione peroxidase (GPx). However, especially in the presence of iron complexes, these ROS may be converted into the more toxic hydroxyl radical $\left(\mathrm{OH}^{\bullet-}\right)$ by several processes within and outside mitochondria (293, 294, 298, 394).

Also, peroxisomes, cytoplasmic organelles specialized for carrying out oxidative reactions, may have a pivotal role in ROS production/regulation in cardiomyocytes. A variety of substrates are broken down by oxidative reactions in peroxisomes, including amino acids, uric acid, and fatty acids. In cardiomyocytes, fatty acid metabolism is very active and peroxisomes are critical for processing long carbon chain fatty acids. Although the role of these organelles in CTX is poorly studied, it is likely that they have a key role in determining the fate of cardiomyocytes that are exposed to cardiotoxic agents (433).

NO is a redox species with both oxidant and antioxidant properties $(294,394)$. NO responses in tumor biology display a biphasic nature (323). NO is produced by biologically important enzymes, namely the three isoforms of NOS: the endothelial (eNOS or NOS3) and neuronal (nNOS or NOS1) NOSs, which are constitutively expressed in specific compartments of the cardiomyocytes, and the inducible NOS2 (iNOS), which is induced by pro-inflammatory mediators or by ischemic episodes $(294,394)$. NO can also be produced by other reactions in biological systems, which are collectively termed "non-NOS" processes $(294,298,323)$. Of note, ROS can react with $\mathrm{NO}$ to generate RNS, thus amplifying the production of oxidant compounds, and NOS itself when uncoupled may produce $\operatorname{ROS}(102,294,298,394)$. Snitrosylation (SNO) is the covalent attachment of an NO moiety to a protein thiol group. As recently reviewed (294, 298), SNO is a redox-dependent modification that often exerts an antioxidant effect, shielding critical cysteine residues from oxidation and affecting protein function (Fig. 10).

The toxic effect produced by many anti-cancer drugs is derived from an altered production of ROS/RNS by different cellular components and enzymes (102, 246, 247, 367). For instance, several drugs alter the activity of redox enzymes within and outside the mitochondria, including an impairment of NOSs, respiratory complexes, the Krebs cycle, oxidative phosphorylation, and $\beta$-oxidation. This impairment results in oxidative/nitrosative stress, a reduction in antioxidant capacity, and induction of cell death $(3,102,246,247,367)$. Also, iNOS upregulation may be involved in carcinogenesis (140).

In the next few paragraphs, we analyze the specificity of redox alterations induced by different chemotherapeutics; particular attention is paid to those exerted by ANTs, which for their adverse side-effects and for historic reasons are the cardiotoxic agents most studied, and which are still in use for the therapy of solid and hematological malignancies.

The generation of ROS, with a consequent rise in oxidative stress, has been considered a key event in ANT-induced CTX. ROS are then effectors of membrane lipid peroxidation, vacuolization, irreversible damage, and myocyte replacement by fibrous tissue $(204,219,248-250,333,353$, 376, 434).

ANTs trigger the generation of ROS, affecting the activity of mitochondrial enzyme complexes, NOSs, NAD(P)H oxidases, catalase, and GPx, and leading to oxidative stress and cell injury. ANTs can be quickly converted to unstable metabolites (such as doxorubicin-semiquinone), which, in turn, 


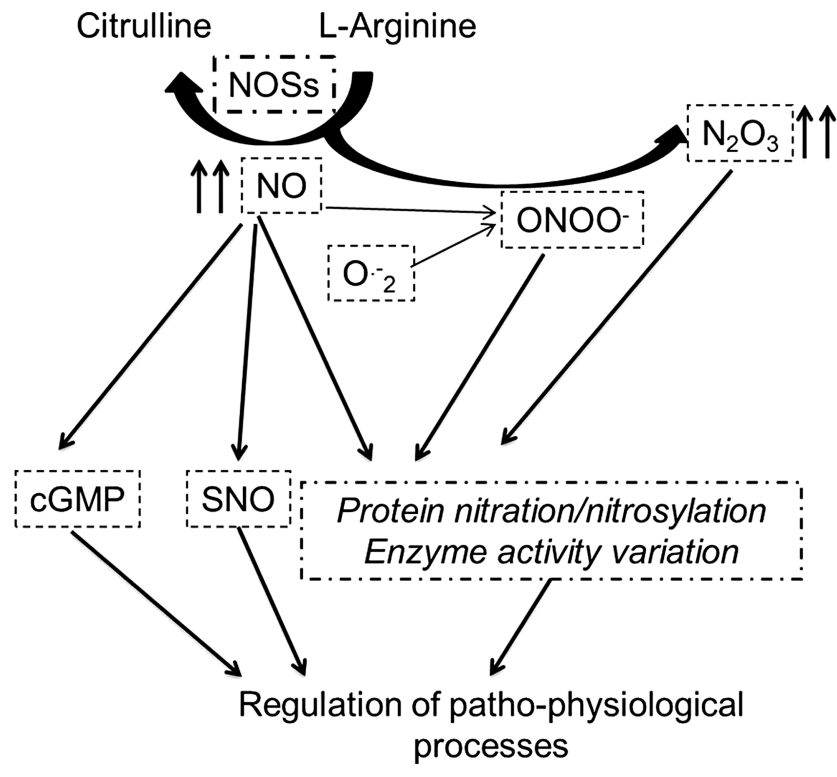

FIG. 10. Antioxidant properties of NO. When production of NO is adequate, the formation of nitrosylating agents may prevail, including $\mathrm{N}_{2} \mathrm{O}_{3}$, which can mediate a direct thiol nitrosylation of proteins, which, in turn, may affect the regulatory function in many physiological processes. See the text for further explanation.

can react with $\mathrm{O}_{2}$, producing $\mathrm{H}_{2} \mathrm{O}_{2}$ and $\mathrm{O}_{2}^{\bullet-}$. In addition, ANTs chelate the free intracellular iron, forming irondoxorubicin complexes. These can react with $\mathrm{O}_{2}$, leading to the generation of ROS. Finally, ANTs are able to directly interfere with the main iron-transporting and -binding proteins $(114,250)$. The Ardehali lab (163) has reported that doxorubicin impairs the expression of ABCB8, a mitochondrial iron exporter, with consequent iron accumulation within these organelles and subsequent ROS generation. Accordingly, overexpression of ABCB8 in isolated cardiomyocytes in vitro, and in the hearts of transgenic mice in vivo, has been proposed as the mechanism protecting from ANT-induced CTX, lowering levels of mitochondrial iron and redox stress. Hearts from patients who developed cardiac dysfunction after ANT treatment exhibit much higher mitochondrial iron levels than normal hearts or hearts affected by different types of dysfunction (163). Such findings support the hypothesis that mitochondrial iron accumulation and oxidative stress play a key role in ANT-induced CTX. Experimental studies have shown that the NRG1/ErbB axis can modulate and counteract the increase in oxidative stress induced by doxorubicin (262, 390), suggesting that CTX from ErbB2 blockade can also involve a dysregulation of redox homeostasis (250).

A portion of cardiac damage induced by ANTs results from their high affinity for cardiolipin, a mitochondrial membrane phospholipid that is involved in apoptotic pathways. The interaction of ANTs with cardiolipin leads to a high concentration of the drug in these organelles. Once accumulated in the mitochondria, the drug may exert adverse effects such as ROS generation, inhibition of oxidative phosphorylation, and mitochondrial DNA damage. During the initial phases, ROS can lead to peroxidation of cardiolipin, which may induce the release of mitochondrial apoptogenic factors, including cytochrome c, which, in turn, can trigger more cardiolipin peroxidation. This leads to a cycle that exacerbates ANT-induced injury. Of note, NO may inhibit the peroxidase activity of cytochrome $\mathrm{c}$ on the cardiolipin complex and can thereby block cardiolipin oxidation. Thus, it may be argued that site-specific and appropriate amounts of NO, which exerts antioxidant properties, may counteract the toxic effects of ANTs $(121,308,413)$.

Although much of ROS generation appears to take place in the mitochondria, soluble oxidoreductases of the cytoplasm, such as the xanthine oxidase and coenzyme of the NAD(P)Hdependent cytochrome $\mathrm{P} 450$ reductases of the ER, may be targeted by ANTs in altering redox equilibrium. For example, doxorubicin deoxyaglycone may be obtained by a reduction process occurring in the ER. Deoxyaglycone may then accumulate in the membranes, where it can alter the function of complex I (NADH dehydrogenase) in membrane mitochondria, or the NOXs, in the plasma membrane (389). Both an excessive production of NO by ANT-induced iNOS and a reduced level of NO, resulting either from the quenching of $\mathrm{NO}$ by the superoxide anion to form peroxynitrite $\left(\mathrm{ONOO}^{-}\right)$ or from uncoupled NOS, have been described as responsible for some of the damage induced by these drugs (103). More studies are required to elucidate the role of such enzymes and of other less-characterized intracellular signaling molecules in ANT-induced CTX. However, these data confirm how the oxidative reactions on the basis of ANT-induced cardiac dysfunction are involved in most kinds of HF, suggesting that timely innovative pharmacological strategies that interfere with specific molecules involved in heart dysfunction, such as p53, may represent a potential common approach in the fight against HF (250).

Oxidative stress may also play a role in CTX derived from TKIs: It has been observed that direct infusion of sunitinib in different experimental preparations provoked a dose-dependent cardiodepressant effect, accompanied by decreased levels of intracellular $\mathrm{Ca}^{2+}$, with a concomitant rise in ROS generation (314).

In addition, 5-FU can also induce oxidative stress in cardiomyocytes and endothelial cells. In particular, the toxic effect exerted by this drug on the vascular endothelium determines eNOS dysregulation, endothelin 1 upregulation, and the activation of protein kinase $\mathrm{C}$. These effects may lead to endothelium-dependent and -independent vasoconstriction, and, subsequently, to coronary spasms $(5,365)$.

\section{A. Drugs with antioxidant properties}

The cardiotoxic mechanisms involving oxidative stress provide the rationale for using drugs with antioxidant properties $(29,34,73,219,224,242$, 350, 366, 379, 381) (Fig. 11).

The iron-chelating drug dexrazoxane has been identified as the most promising cardioprotective strategy in the past years and, up to now, it is the only cardioprotective drug approved by the FDA for ANT-induced CTX. Dexrazoxane is a pro-drug that, once it enters the cardiomyocyte and is rapidly turned into its active form, counteracts the formation of ANT-iron complexes and the subsequent formation of ROS (358) (Table 2). Its efficacy has been extensively tested in multiple randomized trials and two pooled analyses (350, 379 ), and it is confirmed in different subsets of cancer patients $(222,381)$.

It is likely that dexrazoxane possesses unique pharmacodynamic protective mechanisms, because no other iron 


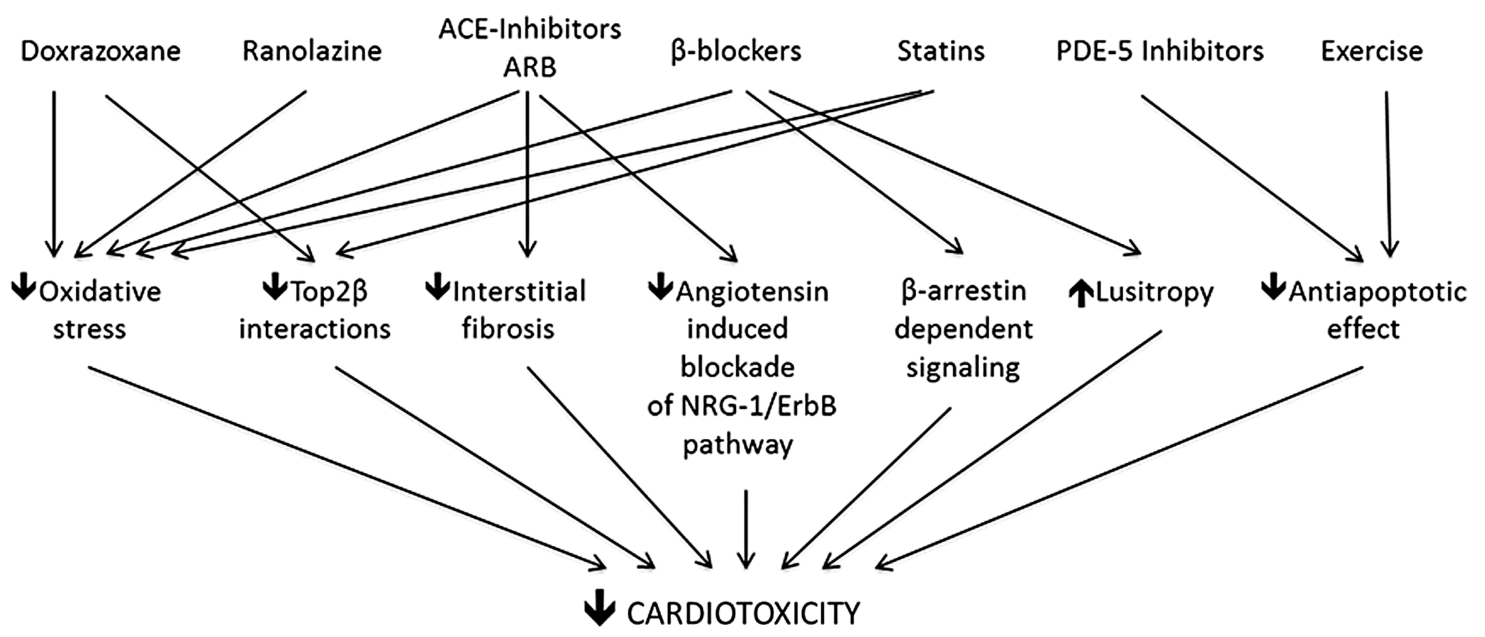

FIG. 11. Drugs and cardioprotective strategies against antineoplastic-induced cardiotoxicity. The main mecha$\operatorname{nism}(\mathrm{s})$ of protection are reported. See the text for further explanation. ACE, angiotensin-converting enzyme; ARB, angiotensin receptor blocker; NRG1, neuregulin 1; Top2, topoisomerase 2.

chelator has shown any cardioprotective effects (358). At the molecular level, dexrazoxane has been shown to modify the Top $2 \beta$ configuration, preventing its interface with ANTs, thereby impeding the realization of the Top2-DNA cleavage complexes $(213,229)$. However, dexrazoxane has been underused in recent years, despite strong evidence of its cardioprotection (367). Although CTX mitigation induced by new-generation $\beta$ blockers could be due more to their antioxidant properties than to their $\beta$-adrenergic receptorblocking action $(243,366,430)$, it appears that the cardioprotective effect of iron chelators does not stem from their antioxidant properties, but rather from their ability to affect iron regulatory proteins and to reduce iron accumulation (42, 114). Interestingly, close derivatives of dexrazoxane lacking Top $2 \beta$ were found not to be protective in relevant chronic ANT CTX models $(178,242)$, which also suggest Top $2 \beta$ importance in the cardioprotective mechanism. These observations indicate that ANT-mediated damage relies on complicated alterations of the intracellular metabolic pathways or Top $2 \beta$ interaction. Moreover, recent preliminary data from Stěrba and coworkers suggest that cardioprotective

Table 2. Drugs Protective Against ANTHRACYCLINE CARDIOTOXICITY

\begin{tabular}{|c|c|c|}
\hline Treatment & Mechanism & Results \\
\hline Dexrazoxane & $\begin{array}{l}\text { ROS reduction } \\
\text { Reduction in DNA damage }\end{array}$ & $\mathrm{HF} \downarrow$ \\
\hline Statins & $\begin{array}{l}\text { Reduction in DNA damage } \\
\text { Reduction in cardiac fibrosis }\end{array}$ & $\mathrm{HF} \downarrow$ \\
\hline ACE inhibitors & $\begin{array}{l}\text { ROS reduction } \\
\text { Reduction in cardiac fibrosis } \\
\text { Downregulation of NRG1/ } \\
\text { ErbB pathway }\end{array}$ & $\mathrm{HF} \downarrow$ \\
\hline$\beta$ blockers & $\begin{array}{l}\text { Downregulation of } \\
\text { catecholamine stimulation } \\
\text { Enhanced lusitropy }\end{array}$ & $\mathrm{HF} \downarrow$ \\
\hline
\end{tabular}

Modified from Madonna et al. (232).

ACE, angiotensin-converting enzyme; NRG1, neuregulin 1; ROS, reactive oxygen species. effects of dexrazoxane against chronic ANT cardiotoxicity are due to its interaction with Top $2 \beta$, rather than to its metalchelating metabolite $(213,372)$. Nevertheless, the discordant results underline the need for further investigation to ascertain the exact biological interaction of anti-cancer drugs with the cellular redox balance.

1. $\beta$ blockers: anti-adrenergic and antioxidant drugs. $\beta$ blockers are a cornerstone therapy in patients with reduced LVEF to prevent symptomatic HF (311), and many researchers encourage their use in asymptomatic ANT-related LV dysfunction $(62,367)$. The rationale for $\beta$ blocker use in ANT-induced CTX rests mostly on experimental indications of their cardioprotective effects. Moreover, a recent retrospective survey in breast cancer patients administered with ANTs and trastuzumab showed that patients who received $\beta$ blockers during their treatment exhibited a significantly lower incidence of HF, supporting the hypothesis that blockage of the $\beta$-adrenergic receptor $(\beta$-AR) may be cardioprotective during antiblastic therapies (347) (Table 2 and Fig. 11).

Long-dated evidence shows $\beta$-AR signaling alterations in ANT-induced cardiomyopathy-like features and in other forms of dilated cardiomyopathies (108), and several animal models have shown a specific positive effect of $\beta$-AR blockage in mitigating oxidative stress, reducing myocardial calcium overload $(14,275)$, and preserving epidermal growth factor signaling $(87,189)$.

The cardioprotective role of the new-generation $\beta$ blockers, such as carvedilol and nebivolol, has also been evaluated. In one study, carvedilol, a nonselective $\beta$ - and $\alpha 1-\mathrm{AR}$ antagonist with strong antioxidant properties, was compared with atenolol, a $\beta$ blocker without antioxidant properties, and showed greater protection against ANT-induced cardiomyopathy; the protective effect of carvedilol, therefore, seems to be related more to its antioxidant properties than to the $\beta$-AR blocking action (243). Carvedilol can prevent ANTinduced ROS release, cardiomyocyte apoptosis (366), and mitochondrial respiration alterations, along with calcium overloading (337). A small, randomized placebo-controlled 
clinical trial on the cardioprotective effect of prophylacticuse carvedilol in patients undergoing ANT chemotherapy showed a reduced incidence of LV systolic and diastolic dysfunction in the carvedilol group (181). However, strong clinical evidence of the cardioprotective effect of carvedilol is still lacking.

Nebivolol is a cardio-selective $\beta$ blocker with limited vasodilating properties related to its interaction with the Larginine/NO pathway. In an experimental model of ANTinduced CTX, treatment with nebivolol, and to a lesser extent with carvedilol, led to an improvement in LV function associated with increased NO levels and a significant reduction in oxidative stress $(71,393,394)$. A small, randomized placebo-controlled study on the prophylactic use of nebivolol in patients undergoing ANT chemotherapy showed, at the 6-month follow-up, a reduced incidence of LV dilatation and systolic dysfunction in the nebivolol group compared with the placebo group (183). In addition, by enhancing prosurvival ERK signaling after ErbB2 inhibition (87), $\beta$ blockers can also prevent trastuzumab-related CTX (Table 3); $\beta$ blockers are now studied in a series of clinical trials such as the MANTICORE 101 and the SAFE trials (33). As said, recent data from the PRADA trial indicated that the $\beta 1$ blocker metoprolol was not cardioprotective (128), thus supporting the use of unbiased $\beta 1$ and $\beta 2$ blockers (382).

In summary, different $\beta$ blockers are not similarly effective in treating CTX. The molecular and cellular mechanisms by which $\beta$ blockers may confer cardioprotection are not completely clear. To date, a cardioprotective treatment with $\beta$ blockers should still demonstrate their efficacy in large clinical trials. In addition, in clinical practice, use of $\beta$ blockers is hampered by their side-effects in an alreadyfragile patient, suggesting their use only in patients with a high CTX risk.

2. The redox role of renin-angiotensin-aldosterone system antagonists: ACE-Is and ARBs. Many studies have found a key role for the renin-angiotensin-aldosterone system (RAAS) in modulating ANT-induced CTX (13). ACE-Is and ARBs have already been shown to exert positive effects in reducing the progression of $\mathrm{LV}$ dysfunction and in preventing $\mathrm{HF}$ in asymptomatic high-risk patients (311).

Table 3. Prevention Strategies for Cardiac Dysfunction Induced by the Main Classes of ANTINEOPLASTIC Drugs

Anticancer agents Prevention strategy Rationale/mechanisms of action

Anthracyclines

Anthracyclines

Lowering of lifetime cumulative doses
Usage of epirubicin, less cardiotoxic
than doxorubicin
Avoiding bolus, preferring infusional
schedules
Liposomal formulations
Dexrazoxane

ACE-1, ARBs

$\beta$ blockers

HMG-CoA reductase inhibitors

Ranolazine
PDE-5 inhibitors
Physical exercise

Anti-ErbB2

ACE-1, ARBs

$\beta$-blockers

Anti-angiogenic drugs (sorafenib and sunitinib)
Anti-hypertensive treatment (with ACE-1 and ARBs, $\beta$ blockers, dihydropyridine calcium channel blockers, diuretics)
Metformin

Dose-dependent cardiotoxicity

Lowering oxidative stress, preventing the interactions between anthracyclines and Top $2 \beta$

Lowering oxidative stress, reducing interstitial fibrosis, enhancing intracellular calcium handling, cardiomyocyte metabolism, and mitochondrial function

Preserving EGF signaling via a $\beta$-arrestindependent mechanism, lowering oxidative stress, improving lusitropy

Inhibiting Top $2 \beta$-mediated DNA damage, antioxidant, and anti-inflammatory effects

Lowering oxidative stress

Anti-apoptotic effects

Lowering oxidative stress and pro-apoptotic signaling, enhancing calcium handling, and enhancing myocardial energetics

Decrease in angiotensin-induced blockade of NRG1/ErbB pathway

Stimulating $\beta$-arrestin-dependent signaling, with subsequest activation of ErbB2-mediated cardioprotective pathways

Counteracting hypertension-dependent cardiac damage

Enhancing myocardial energetics via activation of cAMP-kinase

Modified from Cadeddu et al. (33).

ARBs, angiotensin II receptor blockers; cAMP, cyclic adenosine monophosphate; EGF, epidermal growth factor; HMG-CoA, hydroxymethylglutaryl-coenzyme A; PDE-5, phosphodiesterase-5; Top2, topoisomerase 2. 
The rationale for using RAAS blockade drugs in this setting is based on the high risk of developing myocardial dysfunction after ANT treatment. Experimental studies have shown the efficacy of ACE-Is in countering ANTinduced CTX, both acute high dose (1) and single low dose (24). The underlying mechanisms of their positive effect against ANT-related CTX contribute to neutralizing ROS damage, reducing interstitial fibrosis, avoiding intracellular calcium overload, and improving mitochondrial respiration and cardiomyocyte metabolism $(1,24)$ (Table 2 and Fig. 11). Indeed, activation of the AT1R produces direct adverse $\mathrm{CV}$ effects by stimulating NADPH oxidase, which is involved in the genesis of oxidative stress, and initiating MAPK, related to cell growth, inflammation, hypertrophy, and apoptosis (25).

Experimental animal models of acute and chronic ANTinduced cardiomyopathy showed a cardioprotective role for prophylactic administration of enalapril, captopril, and lisinopril (1). In a study of 115 patients treated with ANTs, enalapril was shown to significantly reduce the incidence of LV dysfunction in comparison with a placebo (36).

Candesartan has been shown to treat experimental cardiotoxicity already induced by ANTs (364), whereas preand post-treatment with telmisartan protected against acute doxorubicin-induced cardiotoxicity in rats (164). Telmisartan is the only ARB-modulating peroxisome proliferator-activated receptor- $\gamma$, and, therefore, affecting the bioavailability of NO, and inhibiting inflammatory molecules such as interleukin 6 (IL-6) and tumor necrosis factor (428). In a small prospective study, telmisartan was shown to mitigate subclinical cardiotoxic effects (changes in strain rate) of epirubicin, with IL-6 and ROS increasing significantly in the placebo arm but not in the telmisartan arm (34). Telmisartan could reverse acute (early) EPI-induced myocardial dysfunction and maintain a normal systolic function till the 18-month follow-up $(73,74)$. IL-6 and ROS increased significantly in the placebo arm but did not change in the telmisartan arm. In another study, valsartan showed a cardioprotective effect on acute cardiotoxicity in patients treated with high doses of ANTs (274).

Recent clinical trials have shown that a combination of ACE-Is and $\beta$ blockers is beneficial in treating ANT-induced CTX (23). Many other ongoing clinical trials are evaluating the role of ACE-Is and ARBs as cardiopreventive agents that may act by decreasing angiotensin-induced blockade of the neuregulin 1/ErbB pathway.

Finally, a recent meta-analysis showed that the prophylactic administration of ACE-Is and ARBs in patients on ANT therapy was associated with a reduced relative risk of developing CTX compared with patients on a placebo (180). To date, however, cardioprotective treatment with ACE-Is or ARBs still requires a demonstration of efficacy in large clinical trials.

In addition, in patients with anti-angiogenic drug-induced hypertension, nondihydropyridine calcium channel blockers are not indicated, because of the pharmacokinetic interaction of sorafenib and sunitinib with CYP3A4 $(33,236)$. Thus, acknowledging the other mechanisms that are responsible for sunitinib-induced CTX, that is, disrupted mitochondrial function and impaired myocyte energy homeostasis, ACE-Is and $\beta$ blockers $(85,106)$ could form an effective cardioprotective strategy (Table 3). Unfortunately, no clinical study to date has shown a role for these drugs in preventing sunitinibinduced CTX (33).

\section{B. Novel strategies in cardioprotection against ANT-induced CTX}

Several CV drugs have been tested as potentially effective in counteracting ANT-induced CTX, and particularly statins, ranolazine, and phosphodiesterase-5 inhibitors.

Lovastatin and fluvastatin, hydroxymethylglutaryl-coenzyme A reductase inhibitors, showed a cardioprotective effect in experimental studies $(159,320)$. Of note, these data derive from acute, subacute experimental cardiotoxicity studies and cell-based studies performed on proliferating H9c2 cell lines, not cardiomyocytes. Therefore, effects of ANTs in this setting deserve caution since these drugs possess strong antiproliferative action besides cardiotoxic effects. The effects of the statins on the Top $2 \beta$ have not been directly demonstrated in the studies, just indirectly suggested to be Top $2 \beta$ related. Besides these caveats, LV systolic dysfunction induced by doxorubicin was not affected by lovastatin (142). Only small clinical studies have evaluated the effects of statins in patients treated with ANTs $(48,348)$, reporting only minor positive results. Unfortunately, the study from the Marwick group (348) was a retrospective observational analysis, and even Chotenimitkhun et al. (48) do not appear to have conducted a standard controlled randomized trial. Therefore, larger studies are, therefore, required to confirm these findings.

Late $\mathrm{I}_{\mathrm{Na}}$ inhibition with ranolazine has been proposed as a cardioprotective strategy in several experimental models of cardiac dysfunction $(316,391,394)$. Ranolazine was shown to preserve heart function in mice treated with ANTs by reducing oxidative stress. The inhibition of elevated $\left[\mathrm{Na}^{+}\right]_{i}$ levels with ranolazine would prevent calcium overload and the occurrence of oxidative damage by suppressing ROS generation, offering an advantage over conventional antioxidant strategies that counteract ROS after their production (196). The INTERACT study (259), designed to assess whether ranolazine could prevent ANT-induced diastolic dysfunction, indicated that ranolazine was a promising cardio-oncologic drug, but more convincing data are still required.

Phosphodiesterase-5 inhibitors have also been tested as cardioprotective agents. Sildenafil demonstrated cardioprotective activity against ANT-induced LV dysfunction by opening mitochondrial $\mathrm{K}_{\mathrm{ATP}}$ channels, preserving mitochondrial membrane potential and myofibrillar integrity, and preventing cardiomyocyte apoptosis (100). Tadalafil attenuated ANT-induced cardiomyopathy through NO-mediated increases in cGMP levels $(177,197)$.

Finally, redox mechanisms have also been proposed for the ability of NRG1 to limit antiblastic drug-induced damage in adult rat cardiomyocytes (390).

Another redox compound attracting the interest of researchers and cardio-oncologists is hydrogen sulfide $\left(\mathrm{H}_{2} \mathrm{~S}\right)$, whose role in cancer is still poorly understood. The discoveries that ROS-induced inhibition of cystathionine gamma-lyase, a key enzyme in the synthesis of $\mathrm{H}_{2} \mathrm{~S}$, is involved in ANT-induced CTX in H9c2 cardiomyocytes, and that the administration of exogenous $\mathrm{H}_{2} \mathrm{~S}$ can confer protection against this CTX, have suggested a new tool with antioxidant properties in therapeutic strategies against antineoplastic drug-induced CTX $(33,247,295)$. Further 
research in appropriate animal models is required, as well as multi-centric randomized trials that compare the efficacy of these new compounds with that of already approved cardioprotective drugs.

Experimental studies have shown that sunitinib inhibits AMPK activity, whereas restoration of this enzyme's activity inhibits cell death (186). It has been speculated that promoting AMPK activity with metformin may reduce sunitinib-induced CTX. Although metformin can counter heart dysfunction in different experimental models of cardiac injury $(53,129)$ (Table 3), an in vitro study that focused on sunitinib-related CTX did not find any beneficial effect of metformin (33). As mentioned earlier, a recent study (98) demonstrated that TrkB, a tyrosine kinase receptor, and its endogenous ligand BDNF could directly modulate the cardiac excitationcontraction coupling, independently or in parallel with GPCR-mediated signaling, supporting the concept that inhibition of the tyrosine kinase receptor with antineoplastic drugs can interfere with fundamental pathways, damaging cardiac contractility (106). At the same time, this study highlights $\mathrm{BDNF}$ as an alternative potential therapeutic strategy for HF.

\section{Nonpharmacologic strategies}

Apart from pharmacologic interventions, we should also consider that nutritional supplementation and exercise training may have important antioxidant properties $(8,139$, 192, 345, 346, 359, 369).

Dietary supplementation of antioxidants proved to be able to alleviate doxorubicin cardiotoxicity in animal models (8, 426). However, there is still scant evidence on both quality and sample size, suggesting that some antioxidant supplementations may lower ANT-induced CTX (109). Nonpharmacologic strategies for countering ANT-induced CTX include lifestyle interventions and exercise "prehabilitation," a type of preventive exercise rehabilitation. It has been speculated that aerobic exercise can decrease ROS generation, inhibiting proapoptotic signaling, restoring proper calcium cycling, and stimulating the AMPK pathway, thus improving myocardial energetics (345) (Table 3). In addition, exercise can have a positive impact on $\mathrm{CV}$ risk factors, such as hypertension, high cholesterol and lipids, overweight and obesity, and high blood glucose or diabetes (192). In cancer survivors, a short period of mixed aerobic and resistance exercise builds tolerance and flexibility in physical activity (369).

Although several studies have shown a role for aerobic exercise in countering ANT (343)- and trastuzumab (139)-related CTX, additional work is needed to better characterize the effects of training on antineoplastic drug-induced CTX (346).

\section{Biomarkers of oxidative/nitrosative CTX}

Since the importance of ROS/RNS production as early mediators of chemotherapy-related CTX has been ascertained, we should identify useful circulating biomarkers of oxidative/nitrosative CTX. Biomarkers with redox significance include the metabolomic identification of acetate and succinate (8). A decrease in $\mathrm{NAD}(\mathrm{P}) \mathrm{H}$ :quinone oxidoreductase 1 activity and an increase in ROS generation by $\mathrm{NAD}(\mathrm{P}) \mathrm{H}$ oxidases have been considered early biomarkers of ANT-induced CTX (283). A significant variation in IL-6, IL-6R, and ROS has been observed together with a change in the enzymatic activity of GPx, which was correlated with an early variation in longitudinal systolic function in patients after the administration of epirubicin $(73,74)$.

Other potential CTX markers with redox importance under investigation in cardiology are high-sensitivity C-reactive protein, heart-type fatty acid-binding protein (H-FABP), glycogen phosphorylase BB (GPBB), and circulating microRNAs, especially microRNA-146a (miR-146a). These are considered new potential markers for the diagnosis and risk stratification of acute coronary syndromes, and they may be helpful in the early detection of anti-cancer CTX; however, the clinical data available in the chemotherapy-induced CTX scenario remain insufficient $(131,152-155,371,419)$. For a discussion on other biomarkers, see section XI.D.

\section{Early Detection, Monitoring, and Management of Heart and Vascular Toxicities}

\section{A. Cardioncology: an expanding science for an expanding problem}

Assessing CTX from antineoplastic drugs is crucial. Screening for CTX at a preclinical stage is essential when investigating the effects of TKIs. Many of these drugs cause an increased risk of arrhythmia; thus, most of the preclinical work focuses on hERG ( $\mathrm{K}^{+}$channel). A major limitation of in vitro work is that primary myocyte cultures of animal or human hearts dedifferentiate and die after a short while. Hence, assays based on stem cells and on the use of engineered heart tissue may better reflect in vivo settings. Such experimental studies could integrate assessment of several effects, including those on membrane action potential, calcium cycling, and myofilament function, as well as those on gene expression and cell survival $(69,88,236,376)$.

In clinical practice, most of the methods used to assess heart function are often inaccurate, and they may also mislead physicians to stop potentially lifesaving therapies. Of note, patients with advanced cancer may already exhibit potential confounder symptoms mimicking anti-cancer drugs' side-effects. Moreover, complications such as myocardial ischemia, arterial hypertension, and arrhythmias are readily recognized, but identifying mild $\mathrm{LV}$ dysfunction (especially at a subclinical stage) can be more challenging (376). In this context, a prompt and sensitive monitoring of $\mathrm{CV}$ function plays a pivotal role.

In addition, the time point when CTX can become clinically overt varies substantially. Several antineoplastic drugs (i.e., ANTs) can induce progressive cardiac remodeling and late cardiomyopathy, whereas others (i.e., trastuzumab) can produce transient LV dysfunction without known long-term consequences $(376,432)$. Furthermore, the inability to exactly predict the long-term consequences of tumors leads to a false diagnosis of CV complications. Owing to all these concerns, cardioncology, a new branch of CV sciences, recently developed, with the aim of preventing, identifying, and treating CV complications of cancer therapy, optimizing clinical status for cardiac patients who need potentially cardiotoxic treatment, facilitates in any way cancer therapy in patients with $\mathrm{CV}$ complications and surveilles for late side-effects in cancer survivors.

\section{B. Diagnostic tools for CTX detection}

The most common clinical diagnostic tools for the screening and detection of CTX are biomarkers and cardiac 
imaging, including echocardiography, multiple-gated acquisition (MUGA) scan, and cardiac magnetic resonance (CMR) (Table 4). Nevertheless, an ECG is recommended for all subjects before and during therapies, particularly for detecting arrhythmias and QT interval prolongation. Moreover, subjects on pyrimidine analogs should be monitored closely for cardiac ischemia by regular ECGs, and chemotherapeutic treatments should be withheld if ischemia is observed (432).

\section{LVEF and emerging modalities for assessing myocardial damage: some claryfing points}

LVEF is the most frequently used parameter for assessing myocardial function, and echocardiography and MUGA scans are largely employed in clinical practice for this purpose (40, 41, 62, 171, 206, 234, 299, 307, 376, 432, 438). An LVEF decrease $(>10 \%)$ to a value $<50 \%$ identifies patients with CTX in both imaging modalities $(307,432)$. Compared with echocardiography, MUGA provides the advantage of a high reproducibility, but it is hampered by significant disadvantages, such as cumulative radiation exposure and no data on other cardiac structures $(299,432)$. However, the choice of modalities for an early detection and monitoring of CTX depends on local expertise and availability, being careful to use the same imaging modality with the best reproducibility for continued screening throughout the treatment period (432). Imaging techniques that provide additional relevant clinical information (e.g., right ventricular function, pulmonary pressures, valvular function, pericardial evaluation) and high-quality radiation-free imaging are preferred $(307,432)$. Confirming LVEF decrease by repeated cardiac imaging (preferably by echocardiography) 2-3 weeks after the baseline initial decrease in LVEF is strongly recommended, as well as categorizing LVEF reduction as asymptomatic or symptomatic, and repeating echocardiography measurements during follow-up (432). Repeated MUGA is not recommended as a first-line option, and it should only be conducted if the radiation exposure is less than five $\mathrm{mSv}$ (432).

On the other hand, echocardiographic measurements, though simple, noninvasive, and readily available, are often variable and of a low sensitivity (290), because of the serious damage and myocyte loss, leading to an observable reduction in LVEF $(92,416)$. On these grounds, novel indices of ventricular function are being introduced (37, 146, 150, 206, 386). Speckle tracking echocardiography has shown the highest sensitivity in detecting early alterations in LV function associated with future development of cardiomyopathy $(134,146,306,307,395,438)$. In particular, a global longitudinal strain $(\mathrm{GLS})>15 \%$ relative percentage reduction

Table 4. Assessement of Anti-Cancer Cardiotoxicity

\begin{tabular}{|c|c|c|c|}
\hline Technique & Diagnostic criteria & Advantages & Disadvantages \\
\hline Echocardiography & LVEF: $>10 \%$ decrease with a value & Availability & Reproducibility \\
\hline 1. LVEF & $\begin{array}{l}<50 \% \text { identify patients with } \\
\text { cardiotoxicity }\end{array}$ & Safety & Image quality \\
\hline 2. GLS & $\begin{array}{l}\text { GLS: }>15 \% \text { relative percentage } \\
\text { reduction from baseline may } \\
\text { identify patients at risk for } \\
\text { cardiotoxicity }\end{array}$ & $\begin{array}{l}\text { Comprehensive cardiac } \\
\text { assessment } \\
\text { Ideal for serial evaluations } \\
\text { Low cost } \\
\text { GLS: Sensitivity } \\
\quad \text { (early CTX) }\end{array}$ & $\begin{array}{l}\text { GLS: Intervendor } \\
\quad \text { variability; low specificity }\end{array}$ \\
\hline $\begin{array}{l}\text { Nuclear cardiac } \\
\text { imaging (MUGA) }\end{array}$ & $\begin{array}{l}>10 \% \text { decrease in LVEF with a } \\
\text { value }<50 \% \text { identifies patients } \\
\text { with cardiotoxicity }\end{array}$ & Reproducibility & $\begin{array}{l}\text { Cumulative radiation exposure } \\
\text { No information on other } \\
\text { cardiac structures and } \\
\text { pericardium } \\
\text { Not recommended for } \\
\text { serial examinations }\end{array}$ \\
\hline $\begin{array}{l}\text { Cardiac magnetic } \\
\text { resonance }\end{array}$ & $\begin{array}{l}\text { Useful if other techniques are } \\
\text { nondiagnostic or to confirm the } \\
\text { presence of LV dysfunction in } \\
\text { borderline LVEF value }\end{array}$ & $\begin{array}{l}\text { Accuracy, reproducibility } \\
\text { RV and pericardium } \\
\text { assessment } \\
\text { Detection of edema, } \\
\text { inflammation, and } \\
\text { fibrosis (early and } \\
\text { late CTX) } \\
\text { Arterial stiffness } \\
\text { measurement }\end{array}$ & $\begin{array}{l}\text { Limited availability } \\
\text { Patient adaptation } \\
\text { General contraindications } \\
\text { High cost } \\
\text { Low feasibility for serial } \\
\quad \text { examinations }\end{array}$ \\
\hline $\begin{array}{l}\text { Cardiac biomarkers } \\
\text { Troponin I }\end{array}$ & $\begin{array}{l}\text { Any increase identifies pts who } \\
\text { may benefit from ACE-Is }\end{array}$ & $\begin{array}{l}\text { Accuracy, } \\
\text { reproducibility }\end{array}$ & Poor evidence for subtle rises \\
\hline Hs Troponin I & $\begin{array}{l}\text { New elevation of Hs-Troponin I } \\
\text { predicts LV dysfunction }\end{array}$ & Wide availability & Variability with different assays \\
\hline $\mathrm{BNP}^{\mathrm{a}}$ & & High sensitivity & $\begin{array}{l}\text { Unclear role for routine } \\
\text { surveillance }\end{array}$ \\
\hline
\end{tabular}

Modified from Zamorano et al. (432).

${ }^{a}$ More useful for their negative predictive values.

CTX, cardiotoxicity; BNP, B-type natriuretic peptide; GLS, global longitudinal strain; LVEF, left ventricle ejection fraction; MUGA, multiple-gated acquisition; pts, patients. 
from baseline can identify patients who are at risk for CTX (97, 172, 307, 432).

Other imaging modalities, such as CMR, appear very promising, although their availability is still limited (97, 238, 290, 299, $388,415)$. CMR is the gold-standard technique for LVEF estimation and can identify myocardial edema, inflammation, capillary leak, and fibrosis, enabling an accurate diagnosis of early and late CTX $(239,388,432)$. Other recent strategies include measuring uptake of iodine-124-metaiodobenzylguanidine, a radiolabeled analog of norepinephrine, which is reduced after myocardial insults $(145,239,415)$. In addition, antimyosin antibodies that detect myosin exposed after cardiac damage could be useful $(235,239,401,402)$, whereas the uptake of radiolabeled chemotherapeutics could be used as a predictor of CTX $(18,239,301)$.

\section{Cardiac biomarkers}

Troponins and natriuretic peptides can be correlated with findings from cardiac imaging for the early detection of CTX $(283,292)$. Natriuretic peptide-level elevations were demonstrated to predict the development of future CTX in patients with NHL and with breast cancer $(99,119)$. Persistent B-type natriuretic peptide (BNP) rises, after exposure to ANTs, were more predictive of following LV dysfunction than transient elevations $(327,335)$. However, the utility of NPs for predicting anticancer drug-induced CTX is still controversial. Indeed, several studies did not confirm a prognostic value for BNP levels, especially when this biomarker was used alone $(66,340,378)$. It has been observed that troponins I and T can predict late ANT-induced CTX in children (223) and in adult patients undergoing high-dose chemotherapy. Not only the increase but also the pattern of the elevation, and particularly a persistent increase after 1 month since treatment, added prognostic information. Conversely, persistently negative TnI identified low-risk patients who did not need a strict cardiological follow-up (38). Patients with troponin increase may benefit from early cardioprotective treatment with ACE-Is (36). In patients treated with trastuzumab, especially after previous exposure to ANTs, troponin I elevation can identify those who will develop LV dysfunction and who will not recover in spite of treatments for HF (37). High-sensitivity troponin I is more sensitive in the evaluation of subclinical depletion of cardiac physiologic reserve, and a new elevation of this marker predicted subsequent LV dysfunction in patients receiving a conventional dose of ANTs and/or trastuzumab (203). The combination of high-sensitivity troponin with GLS predicted the risk to develop future cardiotoxicity with increased sensitivity $(93 \%)$ and negative predictive value $(91 \%)$ compared with the evaluation of a single parameter in patients with breast cancer (340). Therefore, "cardiac biomarkers plus cardiac imaging" should be encouraged to detect CTX early. There are still scant data supporting the use of biomarkers to predict future cardiac dysfunction due to immune and targeted cancer therapies. Few data indicated an increase of troponin in subjects administered sunitinib and sorafenib for metastatic renal cell carcinoma, who developed ventricular function impairment (344). Several other biomarkers have been studied or are under investigation to stratify the risk of cardiotoxicity. Myeloperoxidase, an index of oxidative stress, measured after ANT administration, seems to correlate with subsequent development of CTX (203). FABP and GPBB have also been proposed as novel biomarkers of chemotherapy-mediated cardiac damage, which are even more precocious of troponin and BNP $(86,152$, 153). MicroRNAs have emerged as an attractive tool to detect myocardial injury. miR-146a may induce downregulation of ErbB4 and subsequent stimulation of apoptotic pathways. Preclinical studies have shown increased levels of miR-146a after doxorubicin administration (155). However, more trials with long-term follow-up are needed to validate these data.

The use of cardiac biomarkers in the prediction of cardiotoxicity has not only several advantages (accuracy, reproducibility, high sensitivity, wide availability, and relatively low cost) but also limitations, for example, inter-assay variations, insufficient evidence to establish specific cutoffs for enzyme elevations in this setting, and uncertainty in the timing of blood sampling (432). Standardization in the assessment of cardiac toxicity using biomarkers is needed, and multi-center trials are strongly required before establishing more clear recommendation (376). Table 5 summarizes the main biomarkers that are evaluated for the detection of cardiac toxicity.

\section{E. Detection of vascular complications: old and new strategies}

Structural endothelial damage, endothelial dysfunction, acceleration of atherosclerosis, prothrombotic actions, and vascular inflammation are some of the underlying mechanisms of cancer therapy-related changes in vessel function. VEGF inhibitors, TKIs (such as nilotinib, ponatinib, dasanitib), cisplatin, 5-FU, and ANTs can induce hypertension, ischemia, and TE through their endothelial toxicity. CAD, stroke, PAD, $\mathrm{PAH}$, arterial/venous TE disease, and nephrotoxicity are the main cinical presentations of vascular damage by cancer therapy. In particular, nuclear cellular actions by ANTs may adversely modulate collagen turnover and vascular wall

Table 5. Cardiac Biomarkers to Detect Cardiac Toxicity

\begin{tabular}{lll}
\hline Cardiac biomarkers & Typical mechanisms of release & Anti-cancer drugs \\
\hline Troponins (troponin I and T) & $\begin{array}{l}\text { Ischemia, inflammation, and } \\
\text { oxidative stress } \\
\text { Increase in myocardial wall } \\
\text { stress and LV filling pressure } \\
\text { Natriuretic peptides (BNP pro-BNP) }\end{array}$ & $\begin{array}{c}\text { Anthracyclines; anthracycline- } \\
\text { adjuvant trastuzumab } \\
\text { Anthracyclines; various high- } \\
\text { dose chemotherapy protocols } \\
\text { Anthracyclines }\end{array}$ \\
$\begin{array}{l}\text { Fatty acid-binding protein (FABP) } \\
\text { Glycogen phosphorylase BB (GPBB) }\end{array}$ & $\begin{array}{c}\text { Altered homeostasis } \\
\text { Anthacying microRNAs (miR-146a) }\end{array}$ & Anthracyclines (preclinical studies) \\
\hline
\end{tabular}


homeostasis, leading to acute damage (endothelial dysfunction, increase in smooth muscle tone) and chronic damage (atherosclerosis, increased collagen synthesis), and resulting in an impaired CV coupling $(81,84,273)$. Age and CV risk factors may influence the extent of cardiac dysfunction (Fig. 12). VEGF inhibitors increase vascular tone due to a decrease in NO production, increased peripheral resistance from endothelial damage (sloughing) and dysfunction, and capillary rarefaction (96). Cisplatin can induce myocardial ischemia through an increased procoagulant status.

For an early detection of vascular complications, current reccomendations (432) simply suggest monitoring CV risk factors, assessing patient functional status, measuring blood pressure before cancer treatment, and optimizing anthypertensive therapy during treatment with a goal of $<140 / 90 \mathrm{mmHg}$ (particularly in patients treated with VEGF inhibitors). Moreover, the identification of patients with pre-existing CAD in those undergoing 5FU is of paramount importance since this drug substantially increases the risk of developing myocardial ischemia with a vasospam-recognized mechanism. In addition, it should be considered that clinical factors (cancer-, treatment-, and patient related) could be related to an enhanced risk of venous TE in subjects administered TKIs. Nilotinib, ponatinib, and BCR-ABL TKIs used in treating CML are, instead, associated with PAD and stroke. The evaluation of PAD risk at baseline (risk factor assessment, clinical examination, ankle-brachial index measurement) is, therefore, recommended in these patients (432). Moreover, cerebrovascular ultrasound screening is indicated in subjects who are irradiated for head and neck cancer or lymphoma, especially beyond 5 years after irradiation (432).

However, to date, a common standard approach that is useful in clinical practice for early detection of vascular complications such as atherosclerosis is missing.

The assessment of arterial stiffness, which increases early in the atherosclerotic process, to provide vasculotoxic profiles of chemotherapeutic agents seems to be very proimising. These could be useful in the future in improving therapeutic strategies $(84,179,273,425)$. Drafts et al. found, by magnetic resonance imaging of the aorta, an early ( $\sim 3$ months) and abrupt increase of pulse wave velocity, a marker of arterial stiffness, which was dose-, age-, and CV risk factor independent in patients treated with ANTs; furthermore, this

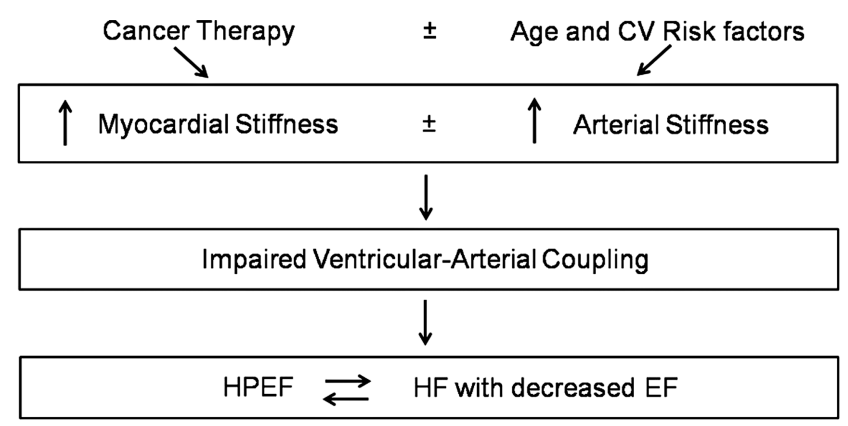

FIG. 12. Simplified algorithm showing how cancer therapy alone or together with demographic and conventional risk factors may determine cardiac damage; both factors can increase myocardial and arterial stiffness, thus impairing ventricular-vascular coupling and, finally, leading to $\mathrm{HF}$, with either decreased ejection fraction (EF) or preserved EF (HFPEF). CV, cardiovascular. increase was accompanied by a subclinical reduction in LVEF and strain (81). This is one of the first demostrations of early atherosclerosis with associated impaired ventriculararterial coupling in cancer survivals. More recently, a marked stiffening of large elastic arteries was demonstrated by applanation tonometry also in patients undergoing VEGF inhibitors (265). Accordingly, ACE-Is should be preferred because of their anti-stiffening properties in the aorta in hypertensive patients treated with ANTs and VEGF inhibitors.

\section{F. Timing of clinical evaluation and follow-up: the importance of risk factors assessment}

To date, no algorithm has been established for evaluation and follow-up of patients before, during, and after cancer therapies. However, a strong collaboration between cardiologists and oncologists is required to monitor and treat oncology patients $(3,432)$. Of course, the cardio-oncology team must be equipped appropriately to address the most common questions (i.e., CTX prediction, prevention strategies, high-risk patient monitoring, management strategies) with an evidencebased approach $(410,425)$.

Long-term surveillance of cancer survivors with a potential for late-onset CV complications should involve cardiooncology teams. According to recent clinical guidelines on cancer treatment and CV toxicity, the first step in cardioprotection should be the identification of subjects at a higher risk; this depends on several factors: cumulative dose, body mass index $>30$ (especially for anti-HER2 compounds), age (elderly and pediatric population treated with ANTs) (199, 376,380 ), concomitant or previous radiation therapy, previous cardiotoxic cancer treatment, pre-existing conditions such as cardiovascular disease (CVD), demographic and other $\mathrm{CV}$ risk factors, and lifestyle and genetic risk factors that may predispose patients to CTX at lower ANT doses (37, 50, 432). Although recent experimental studies from Moulin and collaborators (271) seem to suggest the opposite, female patients appear to be more susceptible to doxorubicin damage (222-224).

Gender-specific pharmacokinetic differences or even a degree of protection conferred by androgens could be responsible for this phenomenon. It has been observed that testosterone can reduce ANT-induced damage in cardiac myocytes in vitro (6).

\section{G. Drug-specific timing for CTX monitoring and treatment}

The exact timing and frequency of imaging and/or biomarker sampling will depend on the specific antineoplastic therapy, total cumulative dose, treatment protocol and duration, and baseline CV risk (432).

1. ANTs. Doxorubicin and epirubicin can induce acute CTX (including ECG changes, supraventricular and ventricular arrhythmias, or transient LV dysfunction), which is usually reversible, develops in $<1 \%$ of patients immediately after infusion of ANTs; early or subacute cardiotoxic effects, occurring within the first year of treatment, and late effects typically are those manifesting after a median of 7 years. Early or subacute CTX, the most frequent, requires a baseline evaluation and further assessment at the end of the treatment, particularly in the presence of an enhanced risk for CTX, or with consecutive treatment with potentially 
cardiotoxic-targeted therapeutics. For higher-dose ANTcontaining regimens, and in subjects with a high baseline risk, earlier evaluation of heart function after a cumulative total doxorubicin (or equivalent) of $240 \mathrm{mg} / \mathrm{m}^{2}$ should be considered (432).

2. Anti-ErbB2. Since patients receiving anti-ErbB2 therapeutics frequently also receive ANTs, a baseline evaluation is always recommended. Usually, cardiac assessment is performed every 3 months during and once after completion of anti-ErbB2 therapies. An improvement in the early detection of cardiac dysfunction with troponins and GLS measurements every 3 months during adjuvant trastuzumab treatment has been demonstrated. Troponin dosage with every cycle may be considered in patients with a high baseline risk (variability in the timing of trastuzumab-induced cardiac dysfunction) (432).

3. VEGF inhibitors. The best timing of surveillance strategies still needs to be clarified. There is high variability in the time of occurrence (early after treatment and delayed for several months). Currently, it is reasonable to consider evaluations every 6 months initially until stability in values of LVEF is achieved. With a high baseline risk, it is better to perform early clinical assessment in the first 2-4 weeks after starting targeted therapies. There is limited evidence to support any specific surveillance strategy. In addition, treatments with anti-VEGF inhibitors (236) pose a high risk $(11 \%-45 \%)$ of inducing new hypertension or destabilizing previously controlled hypertension, including severe hypertension in $2 \%-20 \%$ of cases $(255,432)$. Monitoring blood pressure is, therefore, essential, and susceptible patients should preferably be treated with ACE-Is, ARBs, or $\beta$ blockers instead, considering the beneficial role of these drugs in HF (236, 370, 376).

With regards to therapeutic implications, beyond anthypertensive treatment, eventual standard HF therapy can be administered $(62,376,392)$, and with good life expectancy, aggressive treatments with $\mathrm{LV}$ assist devices can also be considered $(322,376)$. To prevent and treat vascular complications, such as PAD, antiplatelet therapy is recommended (432). Significant stenosis (e.g., those of carotid arteries) may need stenting or surgery. Targeted therapy for PAH can be used temporarily or permanently. Management of QT prolongation is usually dependent on correction of the predisposing factors (e.g., concomitant electrolyte abnormalities, QT-prolonging drugs). Commonly, an individualized approach for the management of atrial fibrillation is needed, and decisions on rate or rhythm control should be patient centred and symptom directed. Anticoagulation for venous TE prevention in patients with cancer should always be considered according to the patient's bleeding risk and life expectancy (432).

\section{Conclusions and Perspectives}

Antineoplastic therapies have led to a prolonged life expectancy for many patients, but CTX is a relatively common adverse effect of these therapies. Apart from direct myocardial toxicity of single drugs, cardiac dysfunction and HF may be exacerbated by drug-drug interactions, which are not only due to treatment with multiple anti-cancer drugs but also due to the disparate drugs that are necessary to mitigate symptoms. Even targeted therapies that were traditionally considered less toxic may exert CTX. Moreover, drugs used to prevent $\mathrm{CV}$ complications have raised some concern about cancer induction (68). Improving the quality of patient care in the CTX setting is, indeed, a complex issue. Despite several proposed strategies to reduce anticancer drug-induced CTX, LV systolic dysfunction and HF still occur in cancer survivors. The scientific community has recognized the complexity of these toxicities, and it is developing multidisciplinary strategies to prevent or minimize CTX and its long-term effects (425). Understanding the mechanisms by which anti-cancer treatments affect the heart is critical for implementing optimal drug design and proposing alternative therapies. Multidisciplinary teams should work toward developing methods to reduce the influence of risk factors and toward detecting and treating potential CTX (3). Early detection requires awareness of chemotherapy-related cardiac dysfunction, as well as appropriate prevention, cardiac evaluation, and follow-up. These aspects are needed to protect individuals who are predisposed to CVD. Very often, many of the patients developing adverse CV events have preexisting known CVD or at least CV risk factors, for which they should receive appropriate treatment and adequate prevention counseling. Measures "that rest on common sense," such as exercise, are recommended for all patients receiving potentially cardiotoxic therapy (367). These measures include the management of preexisting comorbidities and recommendations for a healthy lifestyle, and they should be suggested both before and after initiating cancer therapy. In the absence of specific cardiac monitoring guidelines for potentially cardiotoxic agents, the evaluation and monitoring of LVEF must be considered. However, the prognostic value of serial measurements of LVEF during treatment to detect and monitor CTX remains controversial. Several biomarkers have been proposed, which ideally should be simple to measure, widely available, of a low cost, and extensively used in treating other pathological conditions. For instance, troponin has been proposed for CTX monitoring, but its practical use has yet to be shown definitively $(292,376,432)$.

Hope for progress comes from the development of new promising technologies, including the omics sciences, immunotherapy, investigations of ROS-dependent pathways, and further work involving stem and progenitor cells (250) from both adult and embryonic sources, which so far have been investigated for their capacity to repair and regenerate CV tissues $(233,260)$. Since actively replicating cells are the main target of anti-cancer chemotherapy, stem and progenitor cells may be targeted by drugs that are involved in the onset and development of chemotherapy-induced CTX (20, 260, 363). Specifically, cardiac-derived progenitor or stem cells (CPCs/CSCs) from the adult heart have recently attracted attention as a tool for studying chemotherapy-induced cardiomyopathy and $\mathrm{HF}(67,72)$. CPCs/CSCs that may exist in specific architectural microenvironments known as "cardiac stem cell niches" have very recently entered the clinical trial arena $(233,338)$. Various cell subtypes have been (magnetically) isolated on the basis of specific antibody binding, or preplating in fibronectin-coated culture dishes (yielding cell aggregates known as cardiospheres), therefore producing c-kit ${ }^{+} /$lineage ${ }^{-}$cells, Isl1 cells $(193,208,436)$, and cardiosphere-derived $\mathrm{c}-\mathrm{Kit}^{+} / \mathrm{Sca}-1^{+}$cells. These cells are 
proposed as adult, multipotent stem cells, which are capable of self-renewal in culture, and of differentiating into several cardiac lineages, such as endothelial cells, smooth muscle cells, and cardiomyocytes.

CPCs/CSCs can participate in cardiac regeneration (193, $208,237,252,436)$ and cardioprotection by releasing paracrine factors such as insulin growth factor, granulocyte-colony stimulating factor, VEGF, and stem cell-derived factor- $1 \alpha(20$, $260,319,363)$, although to date there are little data documenting the extent and burden of this process in adults. CPCs/CSCs differentiation may be also responsible for the late-onset ANT-induced CTX, which is an important issue in pediatric oncology $(147,158)$. CPCs/CSCs may be sensitive to interference from a wide range of anti-cancer drugs, resulting in accumulation of oxidative DNA damage, growth arrest, inhibition of CPCs/CSCs proliferation, cell senescence, expression of p53, telomere attrition, and cell necrosis and apoptosis $(67,72)$. In particular, anti-cancer chemotherapy may inhibit the expression of c-kit, resulting in impairment of CPCs/CSCs mobilization in the damaged area (107), and may impair several signaling pathways, including Akt, VEGF, and Notch (251).

Therefore, the idea that autologous CPCs/CSCs can be obtained before antineoplastic drug treatment and used in cancer patients who are sensitive to antineoplastic CTX, for management or prevention of $\mathrm{HF}$, has been proposed (67, 289). Repopulating the damaged heart by boosting endogenous CPCs/CSCs may represent an alternative strategy by which these cells can contribute to myocardial protection, neovascularization, and the reduction of cardiac fibrosis after chemotherapy-induced CTX $(76,360)$. However, since the actual existence of $\mathrm{CPCs} / \mathrm{CSCs}$ and their regenerative capacity have been questioned and are still controversial [with specific reference to the c-kit cells $(375,404-406)]$, further studies are required to clarify several critical questions (e.g., the burden of the endogenous cardiac regenerative process supported by CPCs/CSCs, identification of the molecular mechanisms by which protection, regeneration, and neovascularization may occur), before these approaches are introduced in clinical practice. Indeed, among stem cells, induced pluripotent stem cells are of great interest for research and application in regenerative medicine, disease modeling, drug discovery, and the determination of drug toxicity (83). All these aspects need to be further investigated.

Considering all these perspectives, animal studies that model risk factors for CVD and comorbidities are currently essential for adequate successive clinical translation. Basic and translational studies may help to identify novel and highly effective therapeutic strategies to prevent CTX induced by antincancer therapeutics. The main aim of these studies must be the early identification of such CV alterations, thus preventing their CV side-effects, and the development of more personalized treatments. In this regard, the addition of genetic profiling to clinical risk factor assessment is expected to assist in identifying subjects who are at a high risk of developing CTX in the future. There is an urgent need to close this gap in our knowledge to identify and establish optimal strategies of prevention and management that balance effectiveness of cancer therapy with long-term health $(232,425)$. This is a particularly delicate task, since redox signaling is involved in both mitogenic regulation and tumor suppression (156). Also, on one side, experimental data suggest that antioxidants may be effective in shielding the CV system from toxicity, but on the other side, clinical use of cardioprotective antioxidants is controversial because of the potential reduced cytotoxic efficacy against cancer. Further experimental and clinical studies, in particular well-designed randomized clinical trials, are, therefore, needed to assess this important issue (412).

\section{Acknowledgments}

The authors would like to thank Prof. Donatella Gattullo for her invaluable support, and the Italian Society of Cardiovascular Research, and the Working Group on Drug Cardiotoxicity and Cardioprotection of the Italian Society of Cardiology for having made possible the meeting of scientists who participated in the drafting of this article. The authors are supported by the University of Cagliari (G.M. and C.C.), the University of Torino (Meccasaric to P.P. and C.P.), Regione Campania CISI-Lab, CRÈME Project, TIMING Project (C.G.T. and G.V.), and Ricerca di Ateneo to C.G.T.

\section{References}

1. Abd El-Aziz MA, Othman AI, Amer M, and El-Missiry MA. Potential protective role of angiotensin-converting enzyme inhibitors captopril and enalapril against adriamycin-induced acute cardiac and hepatic toxicity in rats. J Appl Toxicol 21: 469-473, 2001.

2. Aichberger KJ, Herndlhofer S, Schernthaner GH, Schillinger M, Mitterbauer-Hohendanner G, Sillaber C, and Valent P. Progressive peripheral arterial occlusive disease and other vascular events during nilotinib therapy in CML. Am J Hematol 86: 533-539, 2011.

3. Albini A, Pennesi G, Donatelli F, Cammarota R, De Flora S, and Noonan DM. Cardiotoxicity of anticancer drugs: the need for cardio-oncology and cardio-oncological prevention. J Natl Cancer Inst 102: 14-25, 2010.

4. Alhawiti N, Burbury KL, Kwa FA, O'Malley CJ, Shuttleworth P, Alzard M, Hamadi A, Grigg AP, and Jackson DE. The tyrosine kinase inhibitor, nilotinib potentiates a prothrombotic state. Thromb Res 145: 54-64, 2016.

5. Alter P, Herzum M, Soufi M, Schaefer JR, and Maisch B. Cardiotoxicity of 5-fluorouracil. Cardiovasc Hematol Agents Med Chem 4: 1-5, 2006.

6. Altieri P, Barisione C, Lazzarini E, Garuti A, Bezante GP, Canepa M, Spallarossa P, Tocchetti CG, Bollini S, Brunelli $\mathrm{C}$, and Ameri P. Testosterone antagonizes doxorubicininduced senescence of cardiomyocytes. J Am Heart Assoc. 5: pii: e002383, 2016.

7. Aman J, van Bezu J, Damanafshan A, Huveneers S, Eringa EC, Vogel SM, Groeneveld AB, Vonk Noordegraaf A, van Hinsbergh VW, and van Nieuw Amerongen GP. Effective treatment of edema and endothelial barrier dysfunction with imatinib. Circulation 126: 2728-2738, 2012.

8. Andreadou I, Papaefthimiou M, Zira A, Constantinou M, Sigala F, Skaltsounis AL, Tsantili-Kakoulidou A, Iliodromitis EK, Kremastinos DT, and Mikros E. Metabonomic identification of novel biomarkers in doxorubicin cardiotoxicity and protective effect of the natural antioxidant oleuropein. NMR Biomed 22: 585-592, 2009.

9. Anisimov A, Alitalo A, Korpisalo P, Soronen J, Kaijalainen S, Leppänen VM, Jeltsch M, Ylä-Herttuala S, and Alitalo K. Activated forms of VEGF-C and VEGF-D 
provide improved vascular function in skeletal muscle. Circ Res 104: 1302-1312, 2009.

10. Apperley JF. Chronic myeloid leukaemia. Lancet 385: 1447-1459, 2015.

11. Aprile G, Mazzer M, Moroso S, and Puglisi F. Pharmacology and therapeutic efficacy of capecitabine: focus on breast and colorectal cancer. Anticancer Drugs 20: 217-229, 2009.

12. Arbuck SG, Strauss H, Rowinsky E, Christian M, Suffness M, Adams J, Oakes M, McGuire W, Reed E, Gibbs H, Greenfield RA, and Montello M. A reassessment of cardiac toxicity associated with Taxol. J Natl Cancer Inst Monogr (15): 117-130, 1993.

13. Arnolda L, McGrath B, Cocks M, Sumithran E, and Johnston C. Adriamycin cardiomyopathy in the rabbit: an animal model of low output cardiac failure with activation of vasoconstrictor mechanisms. Cardiovasc Res 19: 378$382,1985$.

14. Asanuma H, Minamino T, Sanada S, Takashima S, Ogita H, Ogai A, Asakura M, Liao Y, Asano Y, Shintani Y, Kim J, Shinozaki Y, Mori H, Node K, Kitamura S, Tomoike H, Hori M, and Kitakaze M. Beta-adrenoceptor blocker carvedilol provides cardioprotection via an adenosinedependent mechanism in ischemic canine hearts. Circulation 109: 2773-2779, 2004.

15. Aspeslagh S, Postel-Vinay S, Rusakiewicz S, Soria JC, Zitvogel L, and Marabelle A. Rationale for anti-OX40 cancer immunotherapy. Eur J Cancer 52: 50-66, 2016.

16. Atallah E, Kantarjian H, and Cortes J. In reply to 'Cardiotoxicity of the cancer therapeutic agent imatinib mesylate'. Nat Med 13: 14, 2007.

17. Atrash S, Tullos A, Panozzo S, Bhutani M, Van Rhee F, Barlogie B, and Usmani SZ. Cardiac complications in relapsed and refractory multiple myeloma patients treated with carfilzomib. Blood Cancer J 5: e272, 2015.

18. Behr TM, Behe M, and Wormann B. Trastuzumab and breast cancer. N Engl J Med 345: 995-998, 2001.

19. Belmonte F, Das S, Sysa-Shah P, Sivakumaran V, Stanley B, Guo X, Paolocci N, Aon MA, Nagane M, Kuppusamy $\mathrm{P}$, Steenbergen C, and Abrielson K. ErbB2 overexpression upregulates antioxidant enzymes, reduces basal levels of reactive oxygen species, and protects against doxorubicin cardiotoxicity. Am J Physiol Heart Circ Physiol 309: H1271-H1280, 2015.

20. Beltrami AP, Barlucchi L, Torella D, Baker M, Limana F, Chimenti S, Kasahara H, Rota M, Musso E, Urbanek K, Leri A, Kajstura J, Nadal-Ginard B, and Anversa P. Adult cardiac stem cells are multipotent and support myocardial regeneration. Cell 114: 763-776, 2003.

21. Bloom MW, Hamo CE, Cardinale D, Ky B, Nohria A, Baer L, Skopicki H, Lenihan DJ, Gheorghiade M, Lyon AR, and Butler J. Cancer therapy-related cardiac dysfunction and heart failure: part 1: definitions, pathophysiology, risk factors, and imaging. Circ Heart Fail 9: e002661, 2016.

22. Bocchia M, Galimberti S, Aprile L, Sicuranza A, Gozzini A, Santilli F, Abruzzese E, Baratè C, Scappini B, Fontanelli G, Trawinska MM, Defina M, Gozzetti A, Bosi A, Petrini M, and Puccetti L. Genetic predisposition and induced pro-inflammatory/pro-oxidative status may play a role in increased atherothrombotic events in nilotinib treated chronic myeloid leukemia patients. Oncotarget 7: 72311-72321, 2016.

23. Bosch X, Esteve J, Sitges M, de Caralt TM, Domènech A, Ortiz JT, Monzó M, Morales-Ruiz M, Perea RJ, and Rovira M. Enalapril and carvedilol for preventing chemotherapy-induced left ventricular systolic dysfunction in patients with malignant hemopathies: the OVERCOME trial (preventiOn of left Ventricular dysfunction with Enalapril and caRvedilol in patients submitted to intensive ChemOtherapy for the treatment of Malignant hEmopathies). J Am Coll Cardiol 61: 2355-2362, 2013.

24. Boucek RJ, Steele A, Miracle A, and Atkinson J. Effects of angiotensin-converting enzyme inhibitor on delayedonset doxorubicin-induced cardiotoxicity. Cardiovasc Toxicol 3: 319-329, 2003.

25. Braunwald E. The war against heart failure: the Lancet lecture. Lancet 385: 812-824, 2015.

26. Breccia M, Muscaritoli M, Gentilini F, Latagliata R, Carmosino I, Rossi Fanelli F, and Alimena G. Impaired fasting glucose level as metabolic side effect of nilotinib in non-diabetic chronic myeloid leukemia patients resistant to imatinib. Leuk Res 31: 1770-1772, 2007.

27. Breccia M, Pregno P, Spallarossa P, Arboscello E, Ciceri F, Giorgi M, Grossi A, Mallardo M, Nodari S, Ottolini S, Sala C, Tortorella G, Rosti G, Pane F, Minotti G, and Baccarani M. Identification, prevention and management of cardiovascular risk in chronic myeloid leukaemia patients candidate to ponatinib: an expert opinion. Ann Hematol 96: 549-558, 2017.

28. Brinda BJ, Viganego F, Vo T, Dolan D, and Fradley MG. Anti-VEGF-induced hypertension: a review of pathophysiology and treatment options. Curr Treat Options Cardiovasc Med 18: 33, 2016.

29. Broeyer FJ, Osanto S, Suzuki J, de Jongh F, van Slooten $\mathrm{H}$, Tanis BC, Bruning T, Bax JJ, Ritsema van Eck HJ, de Kam ML, Cohen AF, Mituzhima Y, and Burggraaf J. Evaluation of lecithinized human recombinant super oxide dismutase as cardioprotectant in anthracycline-treated breast cancer patients. Br J Clin Pharmacol 78: 950-960, 2014.

30. Bronte G, Bronte E, Novo G, Pernice G, Lo Vullo F, Musso E, Bronte F, Gulotta E, Rizzo S, Rolfo C, Silvestris N, Bazan V, Novo S, and Russo A. Conquests and perspectives of cardio-oncology in the field of tumor angiogenesis-targeting tyrosine kinase inhibitor-based therapy. Expert Opin Drug Saf 14: 253-267, 2015.

31. Brümmendorf TH, Cortes JE, Khoury HJ, Kantarjian HM, Kim DW, Schafhausen P, Conlan MG, Shapiro M, Turnbull K, Leip E, Gambacorti-Passerini C, and Lipton $\mathrm{JH}$. Factors influencing long-term efficacy and tolerability of bosutinib in chronic phase chronic myeloid leukaemia resistant or intolerant to imatinib. Br J Haematol 172: 97110, 2016.

32. Burstein HJ, Elias AD, Rugo HS, Cobleigh MA, Wolff AC, Eisenberg PD, Lehman M, Adams BJ, Bello CL, DePrimo SE, Baum CM, and Miller KD. Phase II study of sunitinib malate, an oral multitargeted tyrosine kinase inhibitor, in patients with metastatic breast cancer previously treated with an anthracycline and a taxane. J Clin Oncol 26: 1810-1816, 2008.

33. Cadeddu C, Mercurio V, Spallarossa P, Nodari S, Triggiani M, Monte I, Piras R, Madonna R, Pagliaro P, Tocchetti CG, and Mercuro G. Preventing antiblastic drug-related cardiomyopathy: old and new therapeutic strategies. J Cardiovasc Med (Hagerstown) 17: S64-S75, 2016.

34. Cadeddu C, Piras A, Mantovani G, Deidda M, Dessì M, Madeddu C, Massa E, and Mercuro G. Protective effects of the angiotensin II receptor blocker telmisartan on epirubicin-induced inflammation, oxidative stress, and 
early ventricular impairment. Am Heart J 160: 487: e1-e7, 2010.

35. Cardinale D, Colombo A, Bacchiani G, Tedeschi I, Meroni CA, Civelli M, Lamantia G, Colombo N, Cipolla CM, Veglia F, Fiorentini C, and Curigliano G. Early detection of anthracycline cardiotoxicity and improvement with heart failure therapy. Circulation 131: 1981-1988, 2015.

36. Cardinale D, Colombo A, Sandri MT, Lamantia G, Colombo N, Civelli M, Martinelli G, Veglia F, Fiorentini C, and Cipolla CM. Prevention of high-dose chemotherapyinduced cardiotoxicity in high-risk patients by angiotensinconverting enzyme inhibition. Circulation 114: 2474-2481, 2006.

37. Cardinale D, Colombo A, Torrisi R, Sandri MT, Civelli M, Salvatici M, Lamantia G, Colombo N, Cortinovis S, Dessanai MA, Nolè F, Veglia F, and Cipolla CM. Trastuzumab-induced cardiotoxicity: clinical and prognostic implications of troponin I evaluation. J Clin Oncol 28: 3910-3916, 2010.

38. Cardinale D, Sandri MT, Colombo A, Colombo N, Boeri M, Lamantia G, Civelli M, Peccatori F, Martinelli G, Fiorentini C, and Cipolla CM. Prognostic value of troponin I in cardiac risk stratification of cancer patients undergoing high-dose chemotherapy. Circulation 109: 2749-2754, 2004.

39. Carnevale-Schianca F, Gallo S, Rota-Scalabrini D, Sangiolo D, Fizzotti M, Caravelli D, Capaldi A, Anselmetti G, Palesandro E, D'Ambrosio L, Coha V, Obert R, Aglietta M, and Grignani G. Complete resolution of life-threatening bleomycin-induced pneumonitis after treatment with imatinib mesylate in a patient with Hodgkin's lymphoma: hope for severe chemotherapy-induced toxicity? J Clin Oncol 29: e691-e693, 2011.

40. Carver JR, Schuster SJ, and Glick JH. Doxorubicin cardiotoxicity in the elderly: old drugs and new opportunities. J Clin Oncol 26: 3122-3124, 2008.

41. Carver JR, Shapiro CL, Ng A, Jacobs L, Schwartz C, Virgo KS, Hagerty KL, Somerfield MR, and Vaughn DJ; ASCO Cancer Survivorship Expert Panel. American Society of Clinical Oncology clinical evidence review on the ongoing care of adult cancer survivors: cardiac and pulmonary late effects. J Clin Oncol 25: 3991-4008, 2007.

42. Cascales A, Sánchez-Vega B, Navarro N, Pastor-Quirante F, Corral J, Vicente V, and de la Peña FA. Clinical and genetic determinants of anthracycline-induced cardiac iron accumulation. Int J Cardiol 154: 282-286, 2012.

43. Cheng $\mathrm{H}$ and Force $\mathrm{T}$. Molecular mechanisms of cardiovascular toxicity of targeted cancer therapeutics. Circ Res 106: 21-34, 2010.

44. Cheng $\mathrm{H}$ and Force $\mathrm{T}$. Why do kinase inhibitors cause cardiotoxicity and what can be done about it? Prog Cardiovasc Dis 53: 114-120, 2010.

45. Chintalgattu V, Ai D, Langley RR, Zhang J, Bankson JA, Shih TL, Reddy AK, Coombes KR, Daher IN, Pati S, Patel SS, Pocius JS, Taffet GE, Buja LM, Entman ML, and Khakoo AY. Cardiomyocyte PDGFR-beta signaling is an essential component of the mouse cardiac response to load-induced stress. J Clin Invest 120: 472-484, 2010.

46. Chintalgattu V, Rees ML, Culver JC, Goel A, Jiffar T, Zhang J, Dunner K Jr., Pati S, Bankson JA, Pasqualini R, Arap W, Bryan NS, Taegtmeyer H, Langley RR, Yao H, Kupferman ME, Entman ML, Dickinson ME, and Khakoo AY. Coronary microvascular pericytes are the cellular target of sunitinib malate-induced cardiotoxicity. Sci Transl Med 5: 187ra69, 2013.

47. Chislock EM and Pendergast AM. Abl family kinases regulate endothelial barrier function in vitro and in mice. PLoS One 8: e85231, 2013.

48. Chotenimitkhun R, D'Agostino R Jr., Lawrence JA, Hamilton CA, Jordan JH, Vasu S, Lash TL, Yeboah J, Herrington DM, and Hundley WG. Chronic statin administration may attenuate early anthracycline-associated declines in left ventricular ejection function. Can J Cardiol 31: 302-307, 2015.

49. Choueiri TK, Schutz FA, Je Y, Rosenberg JE, and Bellmunt J. Risk of arterial thromboembolic events with sunitinib and sorafenib: a systematic review and metaanalysis of clinical trials. J Clin Oncol 28: 2280-2285, 2010

50. Chow EJ, Chen Y, Kremer LC, Breslow NE, Hudson MM, Armstrong GT, Border WL, Feijen EA, Green DM, Meacham LR, Meeske KA, Mulrooney DA, Ness KK, Oeffinger KC, Sklar CA, Stovall M, van der Pal HJ, Weathers RE, Robison LL, and Yasui Y. Individual prediction of heart failure among childhood cancer survivors. J Clin Oncol 33: 394-402, 2015.

51. Chu TF, Rupnick MA, Kerkela R, Dallabrida SM, Zurakowski D, Nguyen L, Woulfe K, Pravda E, Cassiola F, Desai J, George S, Morgan JA, Harris DM, Ismail NS, Chen JH, Schoen FJ, Van den Abbeele AD, Demetri GD, Force T, and Chen MH. Cardiotoxicity associated with the tyrosine kinase inhibitor sunitinib. Lancet 370: 20112019, 2007.

52. Cianci G, Morelli MF, Cannita K, Morese R, Ricevuto E, Di Rocco ZC, Porzio G, Lanfiuti Baldi P, and Ficorella C. Prophylactic options in patients with 5-fluorouracilassociated cardiotoxicity. Br J Cancer 88: 1507-1509, 2003

53. Cittadini A, Napoli R, Monti MG, Rea D, Longobardi S, Netti PA, Walser M, Samà M, Aimaretti G, Isgaard J, and Saccà L. Metformin prevents the development of chronic heart failure in the SHHF rat model. Diabetes 61: 944 953, 2012.

54. Cortes J, Mauro M, Steegmann JL, Saglio G, Malhotra R, Ukropec JA, and Wallis NT. Cardiovascular and pulmonary adverse events in patients treated with BCR-ABL inhibitors: data from the FDA Adverse Event Reporting System. Am J Hematol 90: E66-E72, 2015.

55. Cortes JE, Jean Khoury H, Kantarjian H, Brümmendorf TH, Mauro MJ, Matczak E, Pavlov D, Aguiar JM, Fly KD, Dimitrov S, Leip E, Shapiro M, Lipton JH, Durand JB, and Gambacorti-Passerini C. Long-term evaluation of cardiac and vascular toxicity in patients with Philadelphia chromosome-positive leukemias treated with bosutinib. Am J Hematol 91: 606-616, 2016.

56. Cortes JE, Kantarjian H, Shah NP, Bixby D, Mauro MJ, Flinn I, O'Hare T, Hu S, Narasimhan NI, Rivera VM, Clackson T, Turner CD, Haluska FG, Druker BJ, Deininger MW, and Talpaz M. Ponatinib in refractory Philadelphia chromosome-positive leukemias. $N$ Engl J Med 367:2075-2088, 2012.

57. Cortes JE, Kantarjian HM, Brümmendorf TH, Kim DW, Turkina AG, Shen ZX, Pasquini R, Khoury HJ, Arkin S, Volkert A, Besson N, Abbas R, Wang J, Leip E, and Gambacorti-Passerini C. Safety and efficacy of bosutinib (SKI-606) in chronic phase Philadelphia chromosome- 
positive chronic myeloid leukemia patients with resistance or intolerance to imatinib. Blood 118: 4567-4576, 2011.

58. Cortes JE, Kim DW, Kantarjian HM, Brümmendorf TH, Dyagil I, Griskevicius L, Malhotra H, Powell C, Gogat K, Countouriotis AM, and Gambacorti-Passerini C. Bosutinib versus imatinib in newly diagnosed chronic-phase chronic myeloid leukemia: results from the BELA trial. J Clin Oncol 30: 3486-3492, 2012.

59. Cortes JE, Kim DW, Pinilla-Ibarz J, le Coutre P, Paquette R, Chuah C, Nicolini FE, Apperley JF, Khoury HJ, Talpaz M, DiPersio J, DeAngelo DJ, Abruzzese E, Rea D, Baccarani M, Müller MC, Gambacorti-Passerini C, Wong S, Lustgarten S, Rivera VM, Clackson T, Turner CD, Haluska FG, Guilhot F, Deininger MW, Hochhaus A, Hughes T, Goldman JM, Shah NP, and Kantarjian H; PACE Investigators. A phase 2 trial of ponatinib in Philadelphia chromosome-positive leukemias. $N$ Engl J Med 369: 1783-1796, 2013.

60. Cortes JE, Saglio G, Kantarjian HM, Baccarani M, Mayer J, Boqué C, Shah NP, Chuah C, Casanova L, BradleyGarelik B, Manos G, and Hochhaus A. Final 5-year study results of DASISION: the dasatinib versus imatinib study in treatment-naïve chronic myeloid leukemia patients trial. J Clin Oncol 34: 2333-2340, 2016.

61. Crone SA, Zhao YY, Fan L, Gu Y, Minamisawa S, Liu Y, Peterson KL, Chen J, Kahn R, Condorelli G, Ross J Jr., Chien KR, and Lee KF. ErbB2 is essential in the prevention of dilated cardiomyopathy. Nat Med 8: 459-465, 2002.

62. Curigliano G, Cardinale D, Suter T, Plataniotis G, de Azambuja E, Sandri MT, Criscitiello C, Goldhirsch A, Cipolla C, and Roila F; ESMO Guidelines Working Group. Cardiovascular toxicity induced by chemotherapy, targeted agents and radiotherapy: ESMO Clinical Practice Guidelines. Ann Oncol 23: vii155-vii166, 2012.

63. D'Uva G, Aharonov A, Lauriola M, Kain D, YahalomRonen Y, Carvalho S, Weisinger K, Bassat E, Rajchman D, Yifa O, Lysenko M, Konfino T, Hegesh J, Brenner O, Neeman M, Yarden Y, Leor J, Sarig R, Harvey RP, and Tzahor E. ERBB2 triggers mammalian heart regeneration by promoting cardiomyocyte dedifferentiation and proliferation. Nat Cell Biol 17: 627-638, 2015.

64. Dahlén T, Edgren G, Lambe M, Höglund M, Björkholm M, Sandin F, Själander A, Richter J, Olsson-Strömberg U, Ohm L, Bäck M, and Stenke L; Swedish CML Group and the Swedish CML Register Group. Cardiovascular events associated with use of tyrosine kinase inhibitors in chronic myeloid leukemia: a population-based cohort study. Ann Intern Med 165: 161-166, 2016.

65. Danhof S, Schreder M, Rasche L, Strifler S, Einsele $\mathrm{H}$, and Knop S. 'Real-life' experience of preapproval carfilzomib-based therapy in myeloma-analysis of cardiac toxicity and predisposing factors. Eur J Haematol 97: 25-32, 2016.

66. Daugaard G, Lassen U, Bie P, Pedersen EB, Jensen KT, Abildgaard U, Hesse B, and Kjaer A. Natriuretic peptides in the monitoring of anthracycline induced reduction in left ventricular ejection fraction. Eur J Heart Fail 7: 8793, 2005.

67. De Angelis A, Piegari E, Cappetta D, Marino L, Filippelli A, Berrino L, Ferreira-Martins J, Zheng H, Hosoda T, Rota M, Urbanek K, Kajstura J, Leri A, Rossi F, and Anversa P. Anthracycline cardiomyopathy is mediated by depletion of the cardiac stem cell pool and is rescued by restoration of progenitor cell function. Circulation 121: 276-292, 2010.

68. De Caterina R. Cancer after intense and prolonged antiplatelet therapies-fact or fiction? Thromb Haemost 114: 1100-1103, 2015.

69. De Keulenaer GW, Doggen K, and Lemmens K. The vulnerability of the heart as a pluricellular paracrine organ: lessons from unexpected triggers of heart failure in targeted ErbB2 anticancer therapy. Circ Res 106: 35-46, 2010.

70. De Korte MA, de Vries EG, Lub-de Hooge MN, Jager PL, Gietema JA, van der Graaf WT, Sluiter WJ, van Veldhuisen DJ, Suter TM, Sleijfer DT, and Perik PJ. 111Indium-trastuzumab visualises myocardial human epidermal growth factor receptor 2 expression shortly after anthracycline treatment but not during heart failure: a clue to uncover the mechanisms of trastuzumab-related cardiotoxicity. Eur J Cancer 43: 2046-2051, 2007.

71. De Nigris F, Rienzo M, Schiano C, Fiorito C, Casamassimi A, and Napoli C. Prominent cardioprotective effects of third generation beta blocker nebivolol against anthracycline-induced cardiotoxicity using the model of isolated perfused rat heart. Eur J Cancer 44: 334-340, 2008.

72. Deidda M, Madonna R, Mango R, Pagliaro P, Bassareo PP, Cugusi L, Romano S, Penco M, Romeo F, and Mercuro G. Novel insights in pathophysiology of antiblastic drugs-induced cardiotoxicity and cardioprotection. $J$ Cardiovasc Med (Hagerstown) 17: S76-S83, 2016.

73. Dessì M, Madeddu C, Piras A, Cadeddu C, Antoni G, Mercuro G, and Mantovani G. Long-term, up to 18 months, protective effects of the angiotensin II receptor blocker telmisartan on Epirubin-induced inflammation and oxidative stress assessed by serial strain rate. Springerplus 2: 198, 2013.

74. Dessì M, Piras A, Madeddu C, Cadeddu C, Deidda M, Massa E, Antoni G, Mantovani G, and Mercuro G. Longterm protective effects of the angiotensin receptor blocker telmisartan on epirubicin-induced inflammation, oxidative stress and myocardial dysfunction. Exp Ther Med 2: 1003-1009, 2011.

75. Devaud C, John LB, Westwood JA, Darcy PK, and Kershaw MH. Immune modulation of the tumor microenvironment for enhancing cancer immunotherapy. Oncoimmunology 2: e25961, 2013.

76. Di GH, Jiang S, Li FQ, Sun JZ, Wu CT, Hu X, and Duan HF. Human umbilical cord mesenchymal stromal cells mitigate chemotherapy-associated tissue injury in a preclinical mouse model. Cytotherapy 14: 412-422, 2012.

77. Di Lorenzo G, Autorino R, Bruni G, Cartenì G, Ricevuto E, Tudini M, Ficorella C, Romano C, Aieta M, Giordano A, Giuliano M, Gonnella A, De Nunzio C, Rizzo M, Montesarchio V, Ewer M, and De Placido S. Cardiovascular toxicity following sunitinib therapy in metastatic renal cell carcinoma: a multicenter analysis. Ann Oncol 20: 1535-1542, 2009.

78. Doggen K, Ray L, Mathieu M, Mc Entee K, Lemmens K, and De Keulenaer GW. Ventricular ErbB2/ErbB4 activation and downstream signaling in pacing-induced heart failure. J Mol Cell Cardiol 46: 33-38, 2009.

79. Dou PQ and Zonder JA. Overview of proteasome inhibitor-based anti-cancer therapies: perspective on bortezomib and second generation proteasome inhibitors versus future generation inhibitors of ubiquitin-proteasome system. Curr Cancer Drug Targets 14: 517-536, 2014. 
80. Douxfils J, Haguet H, Mullier F, Chatelain C, Graux C, and Dogné JM. Association between BCR-ABL tyrosine kinase inhibitors for chronic myeloid leukemia and cardiovascular events, major molecular response, and overall survival: a systematic review and meta-analysis. JAMA Oncol 2: 625-632, 2016.

81. Drafts BC, Twomley KM, D'Agostino R Jr., Lawrence J, Avis N, Ellis LR, Thohan V, Jordan J, Melin SA, Torti FM, Little WC, Hamilton CA, and Hundley WG. Low to moderate dose anthracycline-based chemotherapy is associated with early noninvasive imaging evidence of subclinical cardiovascular disease. JACC Cardiovasc Imaging 6: 877-885, 2013.

82. Dranoff G. Immunotherapy at large: balancing tumor immunity and inflammatory pathology. Nat Med 19: 1100-1111, 2013.

83. Dulak J, Szade K, Szade A, Nowak W, Józkowicz A. Adult stem cells: hopes and hypes of regenerative medicine. Acta Biochim Pol 62: 329-337, 2015.

84. Eckman DM, Stacey RB, Rowe R, D'Agostino R Jr., Kock ND, Sane DC, Torti FM, Yeboah J, Workman S, Lane KS, and Hundley WG. Weekly doxorubicin increases coronary arteriolar wall and adventitial thickness. PLoS One 8: e57554, 2013.

85. Eichhorn EJ, Bedotto JB, Malloy CR, Hatfield BA, Deitchman D, Brown M, Willard JE, and Grayburn PA. Effect of beta-adrenergic blockade on myocardial function and energetics in congestive heart failure. Improvements in hemodynamic, contractile, and diastolic performance with bucindolol. Circulation 82: 473-483, 1990.

86. ElGhandour, El Sorady M, Azab S, and ElRahman M. Human heart-type fatty acid-binding protein as an early diagnostic marker of doxorubicin cardiac toxicity. Hematol Rev 1: e6, 2009.

87. Erickson CE, Gul R, Blessing CP, Nguyen J, Liu T, Pulakat L, Bastepe M, Jackson EK, and Andresen BT. The $\beta$-blocker Nebivolol Is a GRK/ $\beta$-arrestin biased agonist. PLoS One 8: e71980, 2013.

88. Eschenhagen T, Force T, Ewer MS, de Keulenaer GW, Suter TM, Anker SD, Avkiran M, de Azambuja E, Balligand JL, Brutsaert DL, Condorelli G, Hansen A, Heymans S, Hill JA, Hirsch E, Hilfiker-Kleiner D, Janssens S, de Jong S, Neubauer G, Pieske B, Ponikowski P, Pirmohamed M, Rauchhaus M, Sawyer D, Sugden PH, Wojta J, Zannad F, and Shah AM. Cardiovascular side effects of cancer therapies: a position statement from the heart failure association of the European Society of Cardiology. Eur J Heart Fail 13: 1-10, 2011.

89. Eskazan AE, Eyice D, Kurt EA, Elverdi T, Yalniz FF, Salihoglu A, Ar MC, Ongoren Aydin S, Baslar Z, Ferhanoglu B, Aydin Y, Tuzuner N, Ozbek U, and Soysal T. Chronic myeloid leukemia patients who develop grade I/II pleural effusion under second-line dasatinib have better responses and outcomes than patients without pleural effusion. Leuk Res 38: 781-787, 2014.

90. Estabragh ZR, Knight K, Watmough SJ, Lane S, Vinjamuri S, Hart G, and Clark RE. A prospective evaluation of cardiac function in patients with chronic myeloid leukaemia treated with imatinib. Leuk Res 35: 49-51, 2011.

91. Ewer MS and Ewer SM. Troponin I provides insight into cardiotoxicity and the anthracycline-trastuzumab interaction. J Clin Oncol 28: 3901-3904, 2010.
92. Ewer MS and Lenihan DJ. Left ventricular ejection fraction and cardiotoxicity: is our ear really to the ground? J Clin Oncol 26: 1201-1203, 2008.

93. Ewer MS and Lippman SM. Type II chemotherapy-related cardiac dysfunction: time to recognize a new entity. J Clin Oncol 23: 2900-2902, 2005.

94. Ewer MS, Vooletich MT, Durand JB, Woods ML, Davis JR, Valero V, and Lenihan DJ. Reversibility of trastuzumabrelated cardiotoxicity: new insights based on clinical course and response to medical treatment. J Clin Oncol 23: 7820 7826, 2005.

95. Eyre TA and Collins GP. Immune checkpoint inhibition in lymphoid disease. Br J Haematol 170: 291-304, 2015.

96. Facemire CS, Nixon AB, Griffiths R, Hurwitz H, and Coffman TM. Vascular endothelial growth factor receptor 2 controls blood pressure by regulating nitric oxide synthase expression. Hypertension 54: 652-658, 2009.

97. Fallah-Rad N, Walker JR, Wassef A, Lytwyn M, Bohonis S, Fang T, Tian G, Kirkpatrick ID, Singal PK, Krahn M, Grenier D, and Jassal DS. The utility of cardiac biomarkers, tissue velocity and strain imaging, and cardiac magnetic resonance imaging in predicting early left ventricular dysfunction in patients with human epidermal growth factor receptor II-positive breast cancer treated with adjuvant trastuzumab therapy. J Am Coll Cardiol 57: 2263-2270, 2011.

98. Feng N, Huke S, Zhu G, Tocchetti CG, Shi S, Aiba T, Kaludercic N, Hoover DB, Beck SE, Mankowski JL, Tomaselli GF, Bers DM, Kass DA, and Paolocci N. Constitutive BDNF/TrkB signaling is required for normal cardiac contraction and relaxation. Proc Natl Acad Sci U S A 112: 1880-1885, 2015.

99. Feola M, Lombardo E, Taglieri C, Piccolo S, and Vado A. Plasma BNP and renal failure as prognostic factors of mid-term clinical out come in congestive heart failure patients. Int J Cardiol 149: 114-115, 2011.

100. Fisher PW, Salloum F, Das A, Hyder H, and Kukreja RC. Phosphodiesterase-5 inhibition with sildenafil attenuates cardiomyocyte apoptosis and left ventricular dysfunction in a chronic model of doxorubicin cardiotoxicity. Circulation 111: 1601-1610, 2005.

101. Fitter S, Vandyke K, Schultz CG, White D, Hughes TP, and Zannettino AC. Plasma adiponectin levels are markedly elevated in imatinib-treated chronic myeloid leukemia (CML) patients: a mechanism for improved insulin sensitivity in type 2 diabetic CML patients? J Clin Endocrinol Metab 95: 3763-3767, 2010.

102. Fogli S, Nieri P, and Breschi MC. The role of nitric oxide in anthracycline toxicity and prospects for pharmacologic prevention of cardiac damage. FASEB $J$ 18: 664-675, 2004.

103. Folkman J. Angiogenesis: an organizing principle for drug discovery? Nat Rev Drug Discov 6: 273-286, 2007.

104. Folkman J. Tumor angiogenesis: therapeutic implications. $N$ Engl J Med 285: 1182-1186, 1971.

105. Fontanella C, Aita M, Cinausero M, Aprile G, Baldin MG, Dusi V, Lestuzzi C, Fasola G, and Puglisi F. Capecitabineinduced cardiotoxicity: more evidence or clinical approaches to protect the patients' heart? Onco Targets Ther 7; 1783-1791, 2014.

106. Force T and Kolaja KL. Cardiotoxicity of kinase inhibitors: the prediction and translation of preclinical models to clinical outcomes. Nat Rev Drug Discov 10: 111-126, 2011. 
107. Force T, Krause DS, and Van Etten RA. Molecular mechanisms of cardiotoxicity of tyrosine kinase inhibition. Nat Rev Cancer 7: 332-344, 2007.

108. Fu M, Matoba M, Liang QM, Sjögren KG, and Hjalmarson A. Properties of G-protein modulated receptoradenylyl cyclase system in myocardium of spontaneously hypertensive rats treated with adriamycin. Int J Cardiol 44: 9-18, 1994.

109. Fuchs-Tarlovsky V. Role of antioxidants in cancer therapy. Nutrition 29: 15-21, 2013.

110. Gabrielson K, Bedja D, Pin S, Tsao A, Gama L, Yuan B, and Muratore N. Heat shock protein 90 and erbB2 in the cardiac response to doxorubicin injury. Cancer Res 67: 1436-1441, 2007.

111. Galindo CL, Kasasbeh E, Murphy A, Ryzhov S, Lenihan S, Ahmad FA, Williams P, Nunnally A, Adcock J, Song Y, Harrell FE, Tran TL, Parry TJ, Iaci J, Ganguly A, Feoktistov I, Stephenson MK, Caggiano AO, Sawyer DB, and Cleator JH. Anti-remodeling and anti-fibrotic effects of the neuregulin- $1 \beta$ glial growth factor 2 in a large animal model of heart failure. J Am Heart Assoc 3: e000773, 2014.

112. Galindo CL, Ryzhov S, and Sawyer DB. Neuregulin as a heart failure therapy and mediator of reverse remodeling. Curr Heart Fail Rep 11: 40-49, 2014.

113. Gambacorti-Passerini C, Tornaghi L, Franceschino A, Piazza R, Corneo G, and Pogliani E. In reply to 'Cardiotoxicity of the cancer therapeutic agent imatinib mesylate'. Nat Med 13: 13-14, 2007.

114. Gammella E, Maccarinelli F, Buratti P, Recalcati S, and Cairo G. The role of iron in anthracycline cardiotoxicity. Front Pharmacol 5: 25, 2014.

115. Gangadhar TC and Vonderheide RH. Mitigating the toxic effects of anticancer immunotherapy. Nat Rev Clin Oncol 11: 91-99, 2014.

116. Gao R, Zhang J, Cheng L, Wu X, Dong W, Yang X, Li T, Liu X, Xu Y, Li X, and Zhou M. A phase II, randomized, double-blind, multicenter, based on standard therapy, placebo-controlled study of the efficacy and safety of recombinant human neuregulin-1 in patients with chronic heart failure. J Am Coll Cardiol 55: 1907-1914, 2010.

117. Geisler BP, Raad RA, Esaian D, Sharon E, and Schwartz DR. Apical ballooning and cardiomyopathy in a melanoma patient treated with ipilimumab: a case of takotsubo-like syndrome. J Immunother Cancer 3: 4, 2015.

118. Giles FJ, Mauro MJ, Hong F, Ortmann CE, McNeill C, Woodman RC, Hochhaus A, le Coutre PD, and Saglio G. Rates of peripheral arterial occlusive disease in patients with chronic myeloid leukemia in the chronic phase treated with imatinib, nilotinib, or non-tyrosine kinase therapy: a retrospective cohort analysis. Leukemia 27: 1310-1315, 2013.

119. Gimeno E, Gómez M, González JR, Comín J, AlvarezLarrán A, Sánchez-González B, Molina L, DomingoDomenech E, Garcia-Pallarols F, Pedro C, Abella E, Vilaplana C, de Sanjosé S, Besses C, and Salar A. NTproBNP: a cardiac biomarker to assess prognosis in nonHodgkin lymphoma. Leuk Res 35: 715-720, 2011.

120. Giordano SH, Booser DJ, Murray JL, Ibrahim NK, Rahman ZU, Valero V, Theriault RL, Rosales MF, Rivera E, Frye D, Ewer M, Ordonez NG, Buzdar AU, and Hortobagyi GN. A detailed evaluation of cardiac toxicity: a phase II study of doxorubicin and one- or three-hour- infusion paclitaxel in patients with metastatic breast cancer. Clin Cancer Res 8: 3360-3368, 2002.

121. Gonzalvez F and Gottlieb E. Cardiolipin: setting the beat of apoptosis. Apoptosis 12: 877-885, 2007.

122. Goodwin R, Ding K, Seymour L, LeMaître A, Arnold A, Shepherd FA, Dediu M, Ciuleanu T, Fenton D, Zukin M, Walde D, Laberge F, Vincent M, Ellis PM, and Laurie SA; NCIC Clinical Trials Group, Kingston, Ontario, Canada. Treatment-emergent hypertension and outcomes in patients with advanced non-small-cell lung cancer receiving chemotherapy with or without the vascular endothelial growth factor receptor inhibitor cediranib: NCIC Clinical Trials Group Study BR24. Ann Oncol 21: 22202226, 2010.

123. Grandin EW, Ky B, Cornell RF, Carver J, and Lenihan DJ. Patterns of cardiac toxicity associated with irreversible proteasome inhibition in the treatment of multiple myeloma. J Card Fail 21: 138-144, 2015.

124. Green MR, Newton MD, and Fancher KM. Off-target effects of BCR-ABL and JAK2 inhibitors. Am J Clin Oncol 39: 76-84, 2016.

125. Greene SJ, Fonarow GC, Vaduganathan M, Khan SS, Butler J, and Gheorghiade M. The vulnerable phase after hospitalization for heart failure. Nat Rev Cardiol 12: 220229, 2015.

126. Gresset SM and Shah SR. Intricacies of bevacizumabinduced toxicities and their management. Ann Pharmacother 43: 490-501, 2009.

127. Guignabert C, Phan C, Seferian A, Huertas A, Tu L, Thuillet R, Sattler C, Le Hiress M, Tamura Y, Jutant EM, Chaumais MC, Bouchet S, Manéglier B, Molimard M, Rousselot P, Sitbon O, Simonneau G, Montani D, and Humbert M. Dasatinib induces lung vascular toxicity and predisposes to pulmonary hypertension. J Clin Invest 126 : 3207-3218, 2016.

128. Gulati G, Heck SL, Ree AH, Hoffmann P, Schulz-Menger J, Fagerland MW, Gravdehaug B, von KnobelsdorffBrenkenhoff F, Bratland A, Storas TH, Hagve TA, Røsjø $\mathrm{H}$, Steine K, Geisler J, and Omland T. Prevention of cardiac dysfunction during adjuvant breast cancer therapy (PRADA): a $2 \times 2$ factorial, randomized, placebocontrolled, double-blind clinical trial of candesartan and metoprolol. Eur Heart J 37: 1671-1680, 2016.

129. Gundewar S, Calvert JW, Jha S, Toedt-Pingel I, Ji SY, Nunez D, Ramachandran A, Anaya-Cisneros M, Tian R, and Lefer DJ. Activation of AMP-activated protein kinase by metformin improves left ventricular function and survival in heart failure. Circ Res 104: 403-411, 2009.

130. Hägerkvist $R$, Sandler $S$, Mokhtari $D$, and Welsh $N$ Amelioration of diabetes by imatinib mesylate (Gleevec): role of beta-cell NF-kappaB activation and anti-apoptotic pre-conditioning. FASEB J 21: 618-628, 2007.

131. Haghikia A, Podewski E, Libhaber E, Labidi S, Fischer D, Roentgen P, Tsikas D, Jordan J, Lichtinghagen R, von Kaisenberg CS, Struman I, Bovy N, Sliwa K, Bauersachs $\mathrm{J}$, and Hilfiker-Kleiner D. Phenotyping and outcome on contemporary management in a German cohort of patients with peripartum cardiomyopathy. Basic Res Cardiol 108: 366, 2013.

132. Hamo CE, Bloom MW, Cardinale D, Ky B, Nohria A, Baer L, Skopicki H, Lenihan DJ, Gheorghiade M, Lyon AR, and Butler J. Cancer therapy-related cardiac dysfunction and heart failure: part 2: prevention, treatment, guidelines, and future directions. Circ Heart Fail 9: e002843, 2016. 
133. Hanahan D and Weinberg RA. Hallmarks of cancer: the next generation. Cell 144: 646-674, 2011.

134. Hare JL, Brown JK, Leano R, Jenkins C, Woodward N, and Marwick TH. Use of myocardial deformation imaging to detect preclinical myocardial dysfunction before conventional measures in patients undergoing breast cancer treatment with trastuzumab. Am Heart J 158: 294-301, 2009.

135. Hasinoff BB and Patel D. The lack of target specificity of small molecule anticancer kinase inhibitors is correlated with their ability to damage myocytes in vitro. Toxicol Appl Pharmacol 249: 132-139, 2010.

136. Hasinoff BB, Patel D, and Wu X. Molecular mechanisms of the cardiotoxicity of the proteasomal-targeted drugs bortezomib and carfilzomib. Cardiovasc Toxicol 2016 [Epub ahead of print]; DOI: 10.1007/s12012-016-9378-7.

137. Hasinoff BB, Patel D, and Wu X. The myocyte-damaging effects of the BCR-ABL1-targeted tyrosine kinase inhibitors increase with potency and decrease with specificity. Cardiovasc Toxicol 2016 [Epub ahead of print]; DOI: 10.1007/s12012-016-9386-7.

138. Hatfield A, Owen S, and Pilot PR. In reply to 'Cardiotoxicity of the cancer therapeutic agent imatinib mesylate'. Nat Med 13: 13, 2007.

139. Haykowsky MJ, Mackey JR, Thompson RB, Jones LW, and Paterson DI. Adjuvant trastuzumab induces ventricular remodeling despite aerobic exercise training. Clin Cancer Res 15: 4963-4967, 2009.

140. Heinecke JL, Ridnour LA, Cheng RY, Switzer CH, Lizardo MM, Khanna C, Glynn SA, Hussain SP, Young HA, Ambs S, and Wink DA. Tumor microenvironment-based feed-forward regulation of NOS2 in breast cancer progression. Proc Natl Acad Sci U S A 111: 6323-6328, 2014.

141. Heinzerling L, Ott PA, Hodi FS, Husain AN, Tajmir-Riahi A, Tawbi H, Pauschinger M, Gajewski TF, Lipson EJ, and Luke JJ. Cardiotoxicity associated with CTLA4 and PD1 blocking immunotherapy. J Immunother Cancer 4: 50, 2016.

142. Henninger $C$, Huelsenbeck $S$, Wenzel $P$, Brand $M$, Huelsenbeck J, Schad A, and Fritz G. Chronic heart damage following doxorubicin treatment is alleviated by lovastatin. Pharmacol Res 91: 47-56, 2015.

143. Herrmann J, Saguner AM, Versari D, Peterson TE, Chade A, Olson M, Lerman LO, and Lerman A. Chronic proteasome inhibition contributes to coronary atherosclerosis. Circ Res 101: 865-874, 2007.

144. Hesser BA, Liang XH, Camenisch G, Yang S, Lewin DA, Scheller R, Ferrara N, and Gerber HP. Down syndrome critical region protein 1 (DSCR1), a novel VEGF target gene that regulates expression of inflammatory markers on activated endothelial cells. Blood 104: 149-158, 2004.

145. Higuchi $\mathrm{T}$ and Schwaiger $\mathrm{M}$. Imaging cardiac neuronal function and dysfunction. Curr Cardiol Rep 8: 131-138, 2006.

146. Ho E, Brown A, Barrett P, Morgan RB, King G, Kennedy MJ, and Murphy RT. Subclinical anthracycline- and trastuzumab-induced cardiotoxicity in the long-term follow-up of asymptomatic breast cancer survivors: a speckle tracking echocardiographic study. Heart 96: 701$707,2010$.

147. Hoch M, Fischer P, Stapel B, Missol-Kolka E, Sekkali B, Scherr M, Favret F, Braun T, Eder M, Schuster-Gossier K, Gossier A, Hilfker A, Balligand JL, Drexler H, and Hilfker-Kleiner D. Erythropoietin preserves the endothe- lial differentiation capacity of cardiac progenitor cells and reduces heart failure during anticancer therapies. Cell Stem Cell 9: 131-143, 2011.

148. Hochhaus A, Saglio G, Hughes TP, Larson RA, Kim DW, Issaragrisil S, le Coutre PD, Etienne G, Dorlhiac-Llacer PE, Clark RE, Flinn IW, Nakamae H, Donohue B, Deng W, Dalal D, Menssen HD, and Kantarjian HM. Long-term benefits and risks of frontline nilotinib vs imatinib for chronic myeloid leukemia in chronic phase: 5-year update of the randomized ENESTnd trial. Leukemia 30: 10441054, 2016

149. Hodi FS, O’Day SJ, McDermott DF, Weber RW, Sosman JA, Haanen JB, Gonzalez R, Robert C, Schadendorf D, Hassel JC, Akerley W, van den Eertwegh AJ, Lutzky J, Lorigan P, Vaubel JM, Linette GP, Hogg D, Ottensmeier $\mathrm{CH}$, Lebbe C, Peschel C, Quirt I, Clark JI, Wolchok JD, Weber JS, Tian J, Yellin MJ, Nichol GM, Hoos A, and Urba WJ. Improved survival with ipilimumab in patients with metastatic melanoma. $N$ Engl J Med 363: 711-723, 2010.

150. Hoffmann R, von Bardeleben S, ten Cate F, Borges AC, Kasprzak J, Firschke C, Lafitte S, Al-Saadi N, KuntzHehner S, Engelhardt M, Becher H, and Vanoverschelde JL. Assessment of systolic left ventricular function: a multicenter comparison of cineventriculography, cardiac magnetic resonance imaging, unenhanced and contrastenhanced echocardiography. Eur Heart J 26: 607-616, 2005.

151. Honton B, Despas F, Dumonteil N, Rouvellat C, Roussel M, Carrie D, Galinier M, Montastruc JL, and Pathak A. Bortezomib and heart failure: case-report and review of the French Pharmacovigilance database. Fundam Clin Pharmacol 28: 349-352, 2014.

152. Horacek JM, Tichy M, Pudil R, and Jebavy L. Glycogen phosphorylase $\mathrm{BB}$ could be a new circulating biomarker for detection of anthracycline cardiotoxicity. Ann Oncol 19: 1656-1657, 2008.

153. Horacek JM, Tichy M, Jebavy L, Pudil R, Ulrychova M, and Maly J. Use of multiple biomarkers for evaluation of anthracycline-induced cardiotoxicity in patients with acut myeloid leukemia. Exp Oncol 30: 157-159, 2008.

154. Horacek JM, Vasatova M, Tichy M, Pudil R, Jebavy L, and Maly J. The use of cardiac biomarkers in detection of cardiotoxicity associated with conventional and high-dose chemotherapy for acute leukemia. Exp Oncol 32: 97-99, 2010.

155. Horie T, Ono K, Nishi H, Nagao K, Kinoshita M, Watanabe S, Kuwabara Y, Nakashima Y, Takanabe-Mori R, Nishi E, Hasegawa K, Kita T, and Kimura T. Acute doxorubicin cardiotoxicity is associated with miR-146ainduced inhibition of the neuregulin-ErbB pathway. Cardiovasc Res 87: 656-664, 2010.

156. Hornsveld M and Dansen TB. The Hallmarks of cancer from a redox perspective. Antioxid Redox Signal 25: 300325, 2016.

157. Hoshino A, Mita Y, Okawa Y, Ariyoshi M, Iwai-Kanai E, Ueyama T, Ikeda K, Ogata T, and Matoba S. Cytosolic p53 inhibits Parkin-mediated mitophagy and promotes mitochondrial dysfunction in the mouse heart. Nat Commun 4: 2308, 2013.

158. Huang C, Zhang X, Ramil JM, Rikka S, Kim L, Lee Y, Gude NA, Thistlethwaite PA, Sussman MA, Gottlieb RA, and Gustafsson AB. Juvenile exposure to anthracyclines impairs cardiac progenitor cell function and vasculariza- 
tion resulting in greater susceptibility to stress-induced myocardial injury in adult mice. Circulation 121: 675$683,2010$.

159. Huelsenbeck J, Henninger C, Schad A, Lackner KJ, Kaina B, and Fritz G. Inhibition of Rac1 signaling by lovastatin protects against anthracycline-induced cardiac toxicity. Cell Death Dis 2: e190, 2011.

160. Hurwitz H, Fehrenbacher L, Novotny W, Cartwright T, Hainsworth J, Heim W, Berlin J, Baron A, Griffing S, Holmgren E, Ferrara N, Fyfe G, Rogers B, Ross R, and Kabbinavar F. Bevacizumab plus irinotecan, fluorouracil, and leucovorin for metastatic colorectal cancer. $N$ Engl $J$ Med 350: 2335-2342, 2004.

161. Hurwitz HI, Saltz LB, Van Cutsem E, Cassidy J, Wiedemann J, Sirzén F, Lyman GH, and Rohr UP. Venous thromboembolic events with chemotherapy plus bevacizumab: a pooled analysis of patients in randomized phase II and III studies. J Clin Oncol 29: 1757-1764, 2011.

162. Hutson TE, Lesovoy V, Al-Shukri S, Stus VP, Lipatov ON, Bair AH, Rosbrook B, Chen C, Kim S, and Vogelzang NJ. Axitinib versus sorafenib as first-line therapy in patients with metastatic renal-cell carcinoma: a randomised open-label phase 3 trial. Lancet Oncol 14: 12871294, 2013.

163. Ichikawa Y, Ghanefar M, Bayeva M, Wu R, Khechaduri A, Naga Prasad SV, Mutharasan RK, Naik TJ, and Ardehali H. Cardiotoxicity of doxorubicin is mediated through mitochondrial iron accumulation. J Clin Invest 124: 617-630, 2014.

164. Iqbal M, Dubey K, Anwer T, Ashish A, and Pillai KK. Protective effects of telmisartan against acute doxorubicininduced cardiotoxicity in rats. Pharmacol Rep 60: 382-390, 2008.

165. Ito H, Miller SC, Billingham ME, Akimoto H, Torti SV, Wade R, Gahlmann R, Lyons G, Kedes L, and Torti FM. Doxorubicin selectively inhibits muscle gene expression in cardiac muscle cells in vivo and in vitro. Proc Natl Acad Sci U S A 87: 4275-4279, 1990.

166. Ito Y, Miyamoto T, Chong Y, Maki T, Akashi K, and Kamimura T. Nilotinib exacerbates diabetes mellitus by decreasing secretion of endogenous insulin. Int J Hematol 9: 135-138, 2013.

167. Iurlo A, Orsi E, Cattaneo D, Resi V, Bucelli C, Orofino N, Sciumè M, Elena C, Grancini V, Consonni D, Orlandi EM, and Cortelezzi A. Effects of first- and secondgeneration tyrosine kinase inhibitor therapy on glucose and lipid metabolism in chronic myeloid leukemia patients: a real clinical problem? Oncotarget 6: 33944 33951, 2015.

168. Izumiya Y, Shiojima I, Sato K, Sawyer DB, Colucci WS, and Walsh K. Vascular endothelial growth factor blockade promotes the transition from compensatory cardiac hypertrophy to failure in response to pressure overload. Hypertension 47: 887-893, 2006.

169. Jabbour A, Hayward CS, Keogh AM, Kotlyar E, McCrohon JA, England JF, Amor R, Liu X, Li XY, Zhou MD, Graham RM, and Macdonald PS. Parenteral administration of recombinant human neuregulin-1 to patients with stable chronic heart failure produces favourable acute and chronic haemodynamic responses. Eur J Heart Fail 13: 83-92, 2011.

170. Jang SW, Ihm SH, Choo EH, Kim OR, Chang K, Park CS, Kim HY, and Seung KB. Imatinib mesylate attenuates myocardial remodeling through inhibition of plateletderived growth factor and transforming growth factor activation in a rat model of hypertension. Hypertension 63: 1228-1234, 2014.

171. Jarfelt M, Kujacic V, Holmgren D, Bjarnason R, and Lannering B. Exercise echocardiography reveals subclinical cardiac dysfunction in young adult survivors of childhood acute lymphoblastic leukemia. Pediatr Blood Cancer 49: 835-840, 2007.

172. Jassal DS, Han SY, Hans C, Sharma A, Fang T, Ahmadie R, Lytwyn M, Walker JR, Bhalla RS, Czarnecki A, Moussa T, and Singal PK. Utility of tissue Doppler and strain rate imaging in the early detection of trastuzumab and anthracycline mediated cardiomyopathy. J Am Soc Echocardiogr 22: 418-424, 2009.

173. Jemal A, Bray F, Center MM, Ferlay J, Ward E, and Forman D. Global cancer statistics. CA Cancer J Clin 61: 69-90, 2011. Erratum in: CA Cancer J Clin 61: 134, 2011.

174. Jensen SA and Sørensen JB. 5-fluorouracil-based therapy induces endovascular injury having potential significance to development of clinically overt cardiotoxicity. Cancer Chemother Pharmacol 69: 57-64, 2012.

175. Jensen SA and Sørensen JB. Risk factors and prevention of cardiotoxicity induced by 5-fluorouracil or capecitabine. Cancer Chemother Pharmacol 58: 487-493, 2006.

176. Jeon TJ, Lee JD, Ha JW, Yang WI, and Cho SH. Evaluation of cardiac adrenergic neuronal damage in rats with doxorubicin-induced cardiomyopathy using iodine- ${ }^{131}$ MIBG autoradiography and PGP 9.5 immunohistochemistry. Eur J Nucl Med 27: 686-693, 2000.

177. Jin Z, Zhang J, Zhi H, Hong B, Zhang S, Guo H, and Li L. Beneficial effects of tadalafil on left ventricular dysfunction in doxorubicin-induced cardiomyopathy. $J$ Cardiol 62: 110-116, 2013.

178. Jirkovská-Vávrová A, Roh J, Lenčová-Popelová O, Jirkovský E, Hrušková, K, Potůčková-Macková E, Jansová H, Hašková P, Martinková P, Eisner T, Kratochvíl M, Šůs J, Macháček M, Vostatková-Tichotová L, Geršl V, Kalinowski DS, Muller MT, Richardson DR, Vávrová K, Štěrba M, and Šimůnek T. Synthesis and analysis of novel analogues of dexrazoxane and its open-ring hydrolysis product for protection against anthracycline cardiotoxicity in vitro and in vivo. Toxicol Res 4: 10981114, 2015.

179. Jones LM, Stoner L, Brown C, Baldi C, and McLaren B. Cardiovascular disease among breast cancer survivors: the call for a clinical vascular health toolbox. Breast Cancer Res Treat 142: 645-653, 2013.

180. Kalam K and Marwick TH. Role of cardioprotective therapy for prevention of cardiotoxicity with chemotherapy: a systematic review and meta-analysis. Eur J Cancer 49: 2900-2909, 2013.

181. Kalay N, Basar E, Ozdogru I, Er O, Cetinkaya Y, Dogan A, Inanc T, Oguzhan A, Eryol NK, Topsakal R, and Ergin A. Protective effects of carvedilol against anthracyclineinduced cardiomyopathy. J Am Coll Cardiol 48: 22582262, 2006.

182. Kantarjian H, Giles F, Wunderle L, Bhalla K, O'Brien S, Wassmann B, Tanaka C, Manley P, Rae P, Mietlowski W, Bochinski K, Hochhaus A, Griffin JD, Hoelzer D, Albitar M, Dugan M, Cortes J, Alland L, and Ottmann OG. Nilotinib in imatinib-resistant CML and Philadelphia chromosome-positive ALL. N Engl J Med 354: 25422551, 2006. 
183. Kaya MG, Ozkan M, Gunebakmaz O, Akkaya H, Kaya EG, Akpek M, Kalay N, Dikilitas M, Yarlioglues M, Karaca H, Berk V, Ardic I, Ergin A, and Lam YY. Protective effects of nebivolol against anthracycline-induced cardiomyopathy: a randomized control study. Int J Cardiol 167: 2306-2310, 2013.

184. Kelly K, Swords R, Mahalingam D, Padmanabhan S, and Giles FJ. Serosal inflammation (pleural and pericardial effusions) related to tyrosine kinase inhibitors. Target Oncol 4: 99-105, 2009.

185. Kerkelä R, Grazette L, Yacobi R, Iliescu C, Patten R, Beahm C, Walters B, Shevtsov S, Pesant S, Clubb FJ, Rosenzweig A, Salomon RN, Van Etten RA, Alroy J, Durand JB, and Force T. Cardiotoxicity of the cancer therapeutic agent imatinib mesylate. Nat Med 12: 908916, 2006.

186. Kerkela R, Woulfe KC, Durand JB, Vagnozzi R, Kramer D, Chu TF, Beahm C, Chen MH, and Force T. Sunitinibinduced cardiotoxicity is mediated by off-target inhibition of AMP-activated protein kinase. Clin Transl Sci 2: 1525, 2009.

187. Khakoo AY, Kassiotis CM, Tannir N, Plana JC, Halushka M, Bickford C, Trent J, 2nd, Champion JC, Durand JB, and Lenihan DJ. Heart failure associated with Sunitinib Malate: a multitargeted receptor tyrosine kinase inhibitor. Cancer 112: 2500-2508, 2008.

188. Khan MF, Gottesman S, Boyella R, and Juneman E. Gemcitabine-induced cardiomyopathy: a case report and review of the literature. J Medical Case Reports 8: 220$225,2014$.

189. Kim IM, Tilley DG, Chen J, Salazar NC, Whalen EJ, Violin JD, and Rockman HA. Beta-blockers alprenolol and carvedilol stimulate beta-arrestin-mediated EGFR transactivation. Proc Natl Acad Sci U S A 105: 1455514560, 2008.

190. Kim TD, Rea D, Schwarz M, Grille P, Nicolini FE, Rosti G, Levato L, Giles FJ, Dombret H, Mirault T, Labussière H, Lindhorst R, Haverkamp W, Buschmann I, Dörken B, and le Coutre PD. Peripheral artery occlusive disease in chronic phase chronic myeloid leukemia patients treated with nilotinib or imatinib. Leukemia 27: 1316-1321, 2013.

191. Kinhult S, Albertsson M, Eskilsson J, and Cwikiel M. Antithrombotic treatment in protection against thrombogenic effects of 5-fluorouracil on vascular endothelium: a scanning microscopy evaluation. Scanning 23: 1-8, 2001.

192. Kirkham AA and Davis MK. Exercise prevention of cardiovascular disease in breast cancer survivors. J Oncol 2015: 917606, 2015.

193. Klaus A, Müller M, Schulz H, Saga Y, Martin JF, and Birchmeier $\mathrm{W}$. Wnt $/ \beta$-catenin and Bmp signals control distinct sets of transcription factors in cardiac progenitor cells. Proc Natl Acad Sci U S A 109: 10921-10926, 2012.

194. Kloth JS, Pagani A, Verboom MC, Malovini A, Napolitano C, Kruit WH, Sleijfer S, Steeghs N, Zambelli A, and Mathijssen RH. Incidence and relevance of QTc-interval prolongation caused by tyrosine kinase inhibitors. $\mathrm{Br} J$ Cancer 112: 1011-1016, 2015.

195. Koca D, Salman T, Unek IT, Oztop I, Ellidokuz H, Eren $\mathrm{M}$, and Yilmaz U. Clinical and electrocardiography changes in patients treated with capecitabine. Chemotherapy 57: 381-387, 2011.

196. Kohlhaas M, Liu T, Knopp A, Zeller T, Ong MF, Böhm M, O'Rourke B, and Maack C. Elevated cytosolic Na+ increases mitochondrial formation of reactive oxygen species in failing cardiac myocytes. Circulation 121: 1606-1613, 2010.

197. Koka S, Das A, Zhu SG, Durrant D, Xi L, and Kukreja RC. Long-acting phosphodiesterase-5 inhibitor tadalafil attenuates doxorubicin-induced cardiomyopathy without interfering with chemotherapeutic effect. J Pharmacol Exp Ther 334: 1023-1030, 2010.

198. Kosmas C, Kallistratos MS, Kopterides P, Syrios J, Skopelitis H, Mylonakis N, Karabelis A, and Tsavaris N. Cardiotoxicity of fluoropyrimidines in different schedules of administration: a prospective study. J Cancer Res Clin Oncol 134: 75-82, 2008.

199. Kremer LC, van Dalen EC, Offringa M, and Voûte PA. Frequency and risk factors of anthracycline-induced clinical heart failure in children: a systematic review. Ann Oncol 13: 503-512, 2002.

200. Krummel MF and Allison JP. CD28 and CTLA-4 have opposing effects on the response of $\mathrm{T}$ cells to stimulation. J Exp Med 182: 459-465, 1995.

201. Kubiczkova L, Pour L, Sedlarikova L, Hajek R, and Sevcikova S. Proteasome inhibitors-molecular basis and current perspectives in multiple myeloma. J Cell Mol Med 18: 947-961, 2014.

202. Kuehn HS, Ouyang W, Lo B, Deenick EK, Niemela JE, Avery DT, Schickel JN, Tran DQ, Stoddard J, Zhang Y, Frucht DM, Dumitriu B, Scheinberg P, Folio LR, Frein CA, Price S, Koh C, Heller T, Seroogy CM, Huttenlocher A, Rao VK, Su HC, Kleiner D, Notarangelo LD, Rampertaap Y, Olivier KN, McElwee J, Hughes J, Pittaluga S, Oliveira JB, Meffre E, Fleisher TA, Holland SM, Lenardo MJ, Tangye SG, and Uzel G. Immune dysregulation in human subjects with heterozygous germline mutations in CTLA4. Science 345: 1623-1627, 2014.

203. Ky B, Putt M, Sawaya H, French B, Januzzi JL Jr., Sebag IA, Plana JC, Cohen V, Banchs J, Carver JR, Wiegers SE, Martin RP, Picard MH, Gerszten RE, Halpern EF, Passeri J, Kuter I, and Scherrer-Crosbie M. Early increases in multiple biomarkers predict subsequent cardiotoxicity in patients with breast cancer treated with doxorubicin, taxanes, and trastuzumab. J Am Coll Cardiol 63: 809-816, 2014

204. Ky B, Vejpongsa P, Yeh ET, Force T, and Moslehi JJ. Emerging paradigms in cardiomyopathies associated with cancer therapies. Circ Res 113: 754-764, 2013.

205. Lamberti M, Porto S, Zappavigna S, Addeo E, Marra M, Miraglia N, Sannolo N, Vanacore D, Stiuso P, and Caraglia M. A mechanistic study on the cardiotoxicity of 5-fluorouracil in vitro and clinical and occupational perspectives. Toxicol Lett 227: 151-156, 2014.

206. Lancellotti P, Anker SD, Donal E, Edvardsen T, Popescu BA, Farmakis D, Filippatos G, Habib G, Maggioni AP, Jerusalem G, and Galderisi M. EACVI/HFA Cardiac Oncology Toxicity Registry in breast cancer patients: rationale, study design, and methodology (EACVI/HFA COT Registry)-EURObservational Research Program of the European Society of Cardiology. Eur Heart J Cardiovasc Imaging 16: 466-470, 2015.

207. Laubli H, Balmelli C, Bossard M, Pfister O, Glatz K, and Zippelius A. Acute heart failure due to autoimmune myocarditis under pembrolizumab treatment for metastatic melanoma. J Immunother Cancer 3: 11, 2015.

208. Laugwitz KL, Moretti A, Caron L, Nakano A, and Chien KR. Islet1 cardiovascular progenitors: a single source for heart lineages? Development 135: 193-205, 2008. 
209. Le Coutre P, Rea D, Abruzzese E, Dombret H, Trawinska MM, Herndlhofer S, Dörken B, and Valent P. Severe peripheral arterial disease during nilotinib therapy. $J$ Natl Cancer Inst 103: 1347-1348, 2011.

210. Lebrecht D, Kokkori A, Ketelsen UP, Setzer B, and Walker UA. Tissue-specific mtDNA lesions and radicalassociated mitochondrial dysfunction in human hearts exposed to doxorubicin. J Pathol 207: 436-444, 2005.

211. Lee CS, Cragg M, Glennie M, and Johnson P. Novel antibodies targeting immune regulatory checkpoints for cancer therapy. Br J Clin Pharmacol 76: 233-247, 2013.

212. Lee S, Chen TT, Barber CL, Jordan MC, Murdock J, Desai S, Ferrara N, Nagy A, Roos KP, and Iruela-Arispe ML. Autocrine VEGF signaling is required for vascular homeostasis. Cell 130: 691-703, 2007.

213. Lenčová-Popelová O, Jirkovský E, Jansová H, JirkovskáVávrová A, Vostatková-Tichotová L, Mazurová Y, Adamcová M, Chládek J, Hroch M, Pokorná Z, Geršl V, Šimůnek T, and Štěrba M. Cardioprotective effects of inorganic nitrate/nitrite in chronic anthracycline cardiotoxicity: comparison with dexrazoxane. J Mol Cell Cardiol 91: 92-103, 2016.

214. Lenihan DJ and Kowey PR. Overview and management of cardiac adverse events associated with tyrosine kinase inhibitors. Oncologist 18: 900-908, 2013.

215. Lestuzzi C, Tartuferi L, and Corona G. Capecitabine (and 5 fluorouracil) cardiotoxicity. Metabolic considerations. Breast J 17: 564-565, 2011.

216. Lestuzzi C, Vaccher E, Talamini R, Lleshi A, Meneguzzo N, Viel E, Scalone S, Tartuferi L, Buonadonna A, Ejiofor $\mathrm{L}$, and Schmoll HJ. Effort myocardial ischemia during chemotherapy with 5-fluorouracil: an underestimated risk. Ann Oncol 25: 1059-1064, 2014.

217. Lévy BI. Microvascular plasticity and experimental heart failure. Hypertension 47: 827-829, 2006.

218. Li B, Zheng Z, Wei Y, Wang M, Peng J, Kang T, Huang $\mathrm{X}$, Xiao J, Li $\mathrm{Y}$, and $\mathrm{Li} \mathrm{Z}$. Therapeutic effects of neuregulin-1 in diabetic cardiomyopathy rats. Cardiovasc Diabetol 10: 69, 2011.

219. Li T and Singal PK. Adriamycin-induced early changes in myocardial antioxidant enzymes and their modulation by probucol. Circulation 102: 2105-2110, 2000.

220. Lim CC, Zuppinger C, Guo X, Kuster GM, Helmes M, Eppenberger HM, Suter TM, Liao R, and Sawyer DB. Anthracyclines induce calpain-dependent titin proteolysis and necrosis in cardiomyocytes. J Biol Chem 279: 82908299, 2004.

221. Lim SL, Lam CS, Segers VF, Brutsaert DL, and De Keulenaer GW. Cardiac endothelium-myocyte interaction: clinical opportunities for new heart failure therapies regardless of ejection fraction. Eur Heart J 36: 20502060, 2015.

222. Lipshultz SE, Miller TL, Lipsitz SR, Neuberg DS, Dahlberg SE, Colan SD, Silverman LB, Henkel JM, Franco VI, Cushman LL, Asselin BL, Clavell LA, Athale U, Michon B, Laverdière C, Schorin MA, Larsen E, Usmani N, and Sallan SE; Dana-Farber Cancer Institute Acute Lymphoblastic Leukemia Consortium. Continuous versus bolus infusion of doxorubicin in children with ALL: long-term cardiac outcomes. Pediatrics 130: 10031011, 2012.

223. Lipshultz SE, Rifai N, Sallan SE, Lipsitz SR, Dalton V, Sacks DB, and Ottlinger ME. Predictive value of cardiac troponin $\mathrm{T}$ in pediatric patients at risk for myocardial injury. Circulation 96: 2641-2648, 1997.

224. Lipshultz SE, Scully RE, Lipsitz SR, Sallan SE, Silverman LB, Miller TL, Barry EV, Asselin BL, Athale U, Clavell LA, Larsen E, Moghrabi A, Samson Y, Michon B, Schorin MA, Cohen HJ, Neuberg DS, Orav EJ, and Colan SD. Assessment of dexrazoxane as a cardioprotectant in doxorubicin-treated children with high-risk acute lymphoblastic leukaemia: long-term follow-up of a prospective, randomised, multicentre trial. Lancet Oncol 11: 950-961, 2010.

225. Lipson EJ, Bagnasco SM, Moore J Jr., Jang S, Patel MJ, Zachary AA, Pardoll DM, Taube JM, and Drake CG. Tumor regression and allograft rejection after administration of anti-PD-1. N Engl J Med 374: 896-898, 2016.

226. Lipton JH, Chuah C, Guerci-Bresler A, Rosti G, Simpson D, Assouline S, Etienne G, Nicolini FE, le Coutre P, Clark RE, Stenke L, Andorsky D, Oehler V, Lustgarten S, Rivera VM, Clackson T, Haluska FG, Baccarani M, Cortes JE, Guilhot F, Hochhaus A, Hughes T, Kantarjian HM, Shah NP, Talpaz M, Deininger MW, and EPIC investigators. Ponatinib versus imatinib for newly diagnosed chronic myeloid leukaemia: an international, randomised, open-label, phase 3 trial. Lancet Oncol 17: 612-621, 2016.

227. Liu X, Gu X, Li Z, Li X, Li H, Chang J, Chen P, Jin J, Xi B, Chen D, Lai D, Graham RM, and Zhou M. Neuregulin$1 /$ erbB-activation improves cardiac function and survival in models of ischemic, dilated, and viral cardiomyopathy. J Am Coll Cardiol 48: 1438-1447, 2006.

228. Loges S, Roncal C, and Carmeliet P. Development of targeted angiogenic medicine. J Thromb Haemost 7: 2133, 2009.

229. Lyu YL, Kerrigan JE, Lin CP, Azarova AM, Tsai YC, Ban Y, and Liu LF. Topoisomerase Ilbeta mediated DNA double-strand breaks: implications in doxorubicin cardiotoxicity and prevention by dexrazoxane. Cancer Res 67: 8839-8846, 2007.

230. Ma Y, Yamazaki T, Yang H, Kepp O, Galluzzi L, Zitvogel L, Smyth MJ, and Kroemer G. Tumor necrosis factor is dispensable for the success of immunogenic anticancer chemotherapy. Oncoimmunology 2: e24786, 2013.

231. Madonna R, Cadeddu C, Deidda M, Giricz Z, Madeddu C, Mele D, Monte I, Novo G, Pagliaro P, Pepe A, Spallarossa P, Tocchetti CG, Varga ZV, Zito C, Geng YJ, Mercuro G, and Ferdinandy P. Cardioprotection by gene therapy: a review paper on behalf of the Working Group on Drug Cardiotoxicity and Cardioprotection of the Italian Society of Cardiology. Int J Cardiol 191: 203-210, 2015.

232. Madonna R, Cadeddu C, Deidda M, Mele D, Monte I, Novo G, Pagliaro P, Pepe A, Spallarossa P, Tocchetti CG, Zito $\mathrm{C}$, and Mercuro G. Improving the preclinical models for the study of chemotherapy-induced cardiotoxicity: a Position Paper of the Italian Working Group on Drug Cardiotoxicity and Cardioprotection. Heart Fail Rev 20: 621-631, 2015.

233. Madonna R, Van Laake LW, Davidson SM, Engel FB, Hausenloy DJ, Lecour S, Leor J, Perrino C, Schulz R, Ytrehus K, Landmesser U, Mummery CL, Janssens S, Willerson J, Eschenhagen T, Ferdinandy P, and Sluijter JP. Position Paper of the European Society of Cardiology Working Group Cellular Biology of the Heart: cell-based therapies for myocardial repair and regeneration in ischemic heart disease and heart failure. Eur Heart $J$ 37: 1789-1798, 2016. 
234. Maiello M, Sharma RK, Ciccone MM, Reddy HK, and Palmiero P. Early diagnosis of cardiac toxicity related to antineoplastic treatment. J Cancer Ther 2: 161-166, 2011.

235. Maini CL, Sciuto R, Ferraironi A, Vici P, Tofani A, Festa A, Conti F, and Lopez M. Clinical relevance of radionuclide angiography and antimyosin immunoscintigraphy for risk assessment in epirubicin cardiotoxicity. $J$ Nucl Cardiol 4: 502-508, 1997.

236. Maitland ML, Bakris GL, Black HR, Chen HX, Durand JB, Elliott WJ, Ivy SP, Leier CV, Lindenfeld J, Liu G, Remick SC, Steingart R, and Tang WH; Cardiovascular Toxicities Panel, Convened by the Angiogenesis Task Force of the National Cancer Institute Investigational Drug Steering Committee. Initial assessment, surveillance, and management of blood pressure in patients receiving vascular endothelial growth factor signaling pathway inhibitors. J Natl Cancer Inst 102: 596-604, 2010.

237. Makkar RR, Smith RR, Cheng K, Malliaras K, Thomson LE, Berman D, Czer LS, Marbán L, Mendizabal A, Johnston PV, Russell SD, Schuleri KH, Lardo AC, Gerstenblith G, and Marbán E. Intracoronary cardiospherederived cells for heart re generation after myocardial infarction (CADUCEUS): a prospective, randomised phase 1 trial. Lancet 379: 895-904, 2012.

238. Malm S, Frigstad S, Sagberg E, Larsson H, and Skjaerpe $\mathrm{T}$. Accurate and reproducible measurement of left ventricular volume and ejection fraction by contrast echocardiography: a comparison with magnetic resonance imaging. J Am Coll Cardiol 44: 1030-1035, 2004.

239. Markman TM and Markman M. Cardiotoxicity of antineoplastic agents: what is the present and future role for imaging? Curr Oncol Rep 16: 396, 2014.

240. Marone G and Granata F. Angiogenesis, lymphangiogenesis and clinical implications. Preface. Chem Immunol Allergy 99: XI-XII, 2014.

241. Marquez-Rodas I, Cerezuela P, Soria A, Berrocal A, Riso A, Gonzalez-Cao M, and Martin-Algarra S. Immune checkpoint inhibitors: therapeutic advances in melanoma. Ann Transl Med 3: 267, 2015.

242. Martin E, Thougaard AV, Grauslund M, Jensen PB, Bjorkling F, Hasinoff BB, Tjørnelund J, Sehested M, and Jensen LH. Evaluation of the topoisomerase II-inactive bisdioxopiperazine ICRF-161 as a protectant against doxorubicin-induced cardiomyopathy. Toxicology 255: 72-79, 2009.

243. Matsui H, Morishima I, Numaguchi Y, Toki Y, Okumura $\mathrm{K}$, and Hayakawa T. Protective effects of carvedilol against doxorubicin-induced cardiomyopathy in rats. Life Sci 65: 1265-1274, 1999.

244. May D, Gilon D, Djonov V, Itin A, Lazarus A, Gordon O, Rosenberger C, and Keshet E. Transgenic system for conditional induction and rescue of chronic myocardial hibernation provides insights into genomic programs of hibernation. Proc Natl Acad Sci U S A 105: 282-287, 2008.

245. McGuire WP, Rowinsky EK, Rosenhein NB, Grumbine FC, Ettinger DS, Armstrong DK, and Donehower RC. Taxol: a unique antineoplastic agent with significant activity in advanced ovarian epithelial neoplasms. Ann Intern Med 111: 273-279, 1989.

246. Mele D, Nardozza M, Spallarossa P, Frassoldati A, Tocchetti CG, Cadeddu C, Madonna R, Malagù M, Ferrari R, and Mercuro G. Current views on anthracycline cardiotoxicity. Heart Fail Rev 21: 621-634, 2016.
247. Mele D, Tocchetti CG, Pagliaro P, Madonna R, Novo G, Pepe A, Zito C, Maurea N, and Spallarossa P. Pathophysiology of anthracycline cardiotoxicity. J Cardiovasc Med (Hagerstown) 17: S3-S11, 2016.

248. Menna P, Pax OG, Chello M, Covino E, Salvatorelli E, and Minotti G. Anthracycline cardiotoxicity. Expert Opin Drug Saf 1: S21-S36, 2012.

249. Menna P, Salvatorelli E, and Minotti G. Cardiotoxicity of antitumor drugs. Chem Res Toxicol 21: 978-989, 2008.

250. Mercurio V, Pirozzi F, Lazzarini E, Marone G, Rizzo P, Agnetti G, Tocchetti CG, Ghigo A, and Ameri P. Models of heart failure based on the cardiotoxicity of anticancer drugs. J Card Fail 22: 449-458, 2016.

251. Merino $H$ and Singla DK. Notch-1 mediated cardiac protection following embryonic and induced pluripotent stem cell transplantation in doxorubicin-induced heart failure. PLoS One 9: e101024, 2014.

252. Messina E, De Angelis L, Frati G, Morrone S, Chimenti S, Fiordaliso F, Salio M, Battaglia M, Latronico MV, Coletta M, Vivarelli E, Frati L, Cossu G, and Giacomello A. Isolation and expansion of adult cardiac stem cells from human and murine heart. Circ Res 95: 911-921, 2004.

253. Meyer CC, Calis KA, Burke LB, Walawander CA, and Grasela TH. Symptomatic cardiotoxicity associated with 5-fluorouracil. Pharmacotherapy 17: 729-736, 1997.

254. Michot JM, Bigenwald C, Champiat S, Collins M, Carbonnel F, Postel-Vinay S, Berdelou A, Varga A, Bahleda $\mathrm{R}$, Hollebecque A, Massard C, Fuerea A, Ribrag V, Gazzah A, Armand JP, Amellal N, Angevin E, Noel N, Boutros C, Mateus C, Robert C, Soria JC, Marabelle A, and Lambotte $\mathrm{O}$. Immune-related adverse events with immune checkpoint blockade: a comprehensive review. Eur J Cancer 54: 139-148, 2016.

255. Milan A, Puglisi E, Ferrari L, Bruno G, Losano I, and Veglio F. Arterial hypertension and cancer. Int J Cancer 134: 2269-2277, 2014.

256. Miller KD, Chap LI, Holmes FA, Cobleigh MA, Marcom PK, Fehrenbacher L, Dickler M, Overmoyer BA, Reimann JD, Sing AP, Langmuir V, and Rugo HS. Randomized phase III trial of capecitabine compared with bevacizumab plus capecitabine in patients with previously treated metastatic breast cancer. J Clin Oncol 23: 792-799, 2005.

257. Minotti G, Menna P, Salvatorelli E, Cairo G, and Gianni L. Anthracyclines: molecular advances and pharmacologic developments in antitumor activity and cardiotoxicity. Pharmacol Rev 56: 185-229, 2004.

258. Minotti G, Salvatorelli E, and Menna P. Pharmacological foundations of cardio-oncology. J Pharmacol Exp Ther 334: 2-8, 2010.

259. Minotti G. Pharmacology at work for cardio-oncology: ranolazine to treat early cardiotoxicity induced by antitumor drugs. J Pharmacol Exp Ther 346: 343-349, 2013.

260. Mirotsou M, Jayawardena TM, Schmeckpeper J, Gnecchi $\mathrm{M}$, and Dzau VJ. Paracrine mechanisms of stem cell reparative and regenerative actions in the heart. $\mathrm{J} \mathrm{Mol} \mathrm{Cell}$ Cardiol 50: 280-289, 2011.

261. Miura K, Kinouchi M, Ishida K, Kinouchi M, Ishida K, Fujibuchi W, Naitoh T, Ogawa H, Ando T, Yazaki N, Watanabe K, Haneda S, Shibata C, and Sasaki I. 5-FU metabolism in cancer and orally-administrable 5-FU drugs. Cancers (Basel) 2: 1717-1730, 2010.

262. Molinaro M, Ameri P, Marone G, Petretta M, Abete P, Di Lisa F, De Placido S, Bonaduce D, and Tocchetti CG. Recent advances on pathophysiology, diagnostic and 
therapeutic insights in cardiac dysfunction induced by antineoplastic drugs. Biomed Res Int 2015: 138148, 2015.

263. Montani D, Bergot E, Günther S, Savale L, Bergeron A, Bourdin A, Bouvaist H, Canuet M, Pison C, Macro M, Poubeau P, Girerd B, Natali D, Guignabert C, Perros F, O'Callaghan DS, Jaïs X, Tubert-Bitter P, Zalcman G, Sitbon O, Simonneau G, and Humbert M. Pulmonary arterial hypertension in patients treated by dasatinib. Circulation 125: 2128-2137, 2012.

264. Montani D, Seferian A, Savale L, Simonneau G, and Humbert M. Drug-induced pulmonary arterial hypertension: a recent outbreak. Eur Respir Rev 22: 244-250, 2013.

265. Moreo A, Vallerio P, Ricotta R, Stucchi M, Pozzi M, Musca F, Meani P, Maloberti A, Facchetti R, Di Bella S, Giganti MO, Sartore-Bianchi A, Siena S, Mancia G, and Giannattasio C. Effects of cancer therapy targeting vascular endothelial growth factor receptor on central blood pressure and cardiovascular system. Am J Hypertens 29: 158-162, 2016.

266. Moslehi JJ. Cardiovascular toxic effects of targeted cancer therapies. N Engl J Med 375: 1457-1467, 2016.

267. Moslehi JJ and Deininger M. Tyrosine kinase inhibitorassociated cardiovascular toxicity in chronic myeloid leukemia. J Clin Oncol 33: 4210-4218, 2015.

268. Motzer RJ, Hutson TE, Cella D, Reeves J, Hawkins R, Guo J, Nathan P, Staehler M, de Souza P, Merchan JR, Boleti E, Fife K, Jin J, Jones R, Uemura H, De Giorgi U, Harmenberg U, Wang J, Sternberg CN, Deen K, McCann L, Hackshaw MD, Crescenzo R, Pandite LN, and Choueiri TK. Pazopanib versus sunitinib in metastatic renal-cell carcinoma. N Engl J Med 369: 722-731, 2013.

269. Motzer RJ, Hutson TE, Tomczak P, Michaelson MD, Bukowski RM, Rixe O, Oudard S, Negrier S, Szczylik C, Kim ST, Chen I, Bycott PW, Baum CM, and Figlin RA. Sunitinib versus interferon alfa in metastatic renal-cell carcinoma. N Engl J Med 356: 115-124, 2007.

270. Motzer RJ, Michaelson MD, Redman BG, Hudes GR, Wilding G, Figlin RA, Ginsberg MS, Kim ST, Baum CM, DePrimo SE, Li JZ, Bello CL, Theuer CP, George DJ, and Rini BI. Activity of SU11248, a multitargeted inhibitor of vascular endothelial growth factor receptor and platelet-derived growth factor receptor, in patients with metastatic renal cell carcinoma. J Clin Oncol 24: 16-24, 2006.

271. Moulin M, Piquereau J, Mateo P, Fortin D, Rucker-Martin C, Gressette M, Lefebvre F, Gresikova M, Solgadi A, Veksler V, Garnier A, and Ventura-Clapier R. Sexual dimorphism of doxorubicin-mediated cardiotoxicity: potential role of energy metabolism remodeling. Circ Heart Fail 8: 98-108, 2015.

272. Mourad JJ and Levy BI. Mechanisms of antiangiogenicinduced arterial hypertension. Curr Hypertens Rep 13: 289-293, 2011.

273. Murata T, Yamawaki H, Hori M, Sato K, Ozaki H, and Karaki H. Chronic vascular toxicity of doxorubicin in an organ-cultured artery. Br J Pharmacol 132: 1365-1373, 2001.

274. Nakamae H, Tsumura K, Terada Y, Nakane T, Nakamae M, Ohta K, Yamane T, and Hino M. Notable effects of angiotensin II receptor blocker, valsartan, on acute cardiotoxic changes after standard chemotherapy with cyclophosphamide, doxorubicin, vincristine, and prednisolone. Cancer 104: 2492-2498, 2005.
275. Nakamura K, Kusano K, Nakamura Y, Kakishita M, Ohta K, Nagase S, Yamamoto M, Miyaji K, Saito H, Morita H, Emori T, Matsubara H, Toyokuni S, and Ohe T. Carvedilol decreases elevated oxidative stress in human failing myocardium. Circulation 105: 2867-2871, 2002.

276. Naymagon L and Abdul-Hay M. Novel agents in the treatment of multiple myeloma: a review about the future. J Hematol Oncol J 9: 52, 2016.

277. Nazer B, Humphreys BD, and Moslehi J. Effects of novel angiogenesis inhibitors for the treatment of cancer on the cardiovascular system: focus on hypertension. Circulation 124: 1687-1691, 2011.

278. Ng M, Cunningham D, and Norman AR. The frequency and pattern of cardiotoxicity observed with capecitabine used in conjunction with oxaliplatin in patients treated for advanced colorectal cancer (CRC). Eur J Cancer 41: 1542-1546, 2005.

279. Nguyen LT and Ohashi PS. Clinical blockade of PD1 and LAG3-potential mechanisms of action. Nat Rev Immunol 15: 45-56, 2015.

280. Nishimura H, Okazaki T, Tanaka Y, Nakatani K, Hara M, Matsumori A, Sasayama S, Mizoguchi A, Hiai H, Minato $\mathrm{N}$, and Honjo T. Autoimmune dilated cardiomyopathy in PD-1 receptor-deficient mice. Science 291: 319-322, 2001.

281. Nohria A. $\beta$-Adrenergic blockade for anthracycline- and trastuzumab-induced cardiotoxicity: is prevention better than cure? Circ Heart Fail 6: 358-361, 2013.

282. Nousiainen T, Vanninen E, Jantunen E, Remes J, Ritanen E, Vuolteenaho O, and Hartikainen J. Neuroendocrine changes during the evolution of doxorubicin-induced left ventricular dysfunction in adult lymphoma patients. Clin Sci (Lond) 101: 601-607, 2001.

283. Novo G, Cadeddu C, Sucato V, Pagliaro P, Romano S, Tocchetti CG, Zito C, Longobardo L, Nodari S, and Penco $\mathrm{M}$. Role of biomarkers in monitoring antiblastic cardiotoxicity. J Cardiovasc Med (Hagerstown) 17: S27-S34, 2016.

284. Nowis D, Mączewski M, Mackiewicz U, Kujawa M, Ratajska A, Wieckowski MR, Wilczyński GM, Malinowska M, Bil J, Salwa P, Bugajski M, Wójcik C, Siński M, Abramczyk P, Winiarska M, Dabrowska-Iwanicka A, Duszyński J, Jakóbisiak M, and Golab J. Cardiotoxicity of the anticancer therapeutic agent bortezomib. Am J Pathol 176: 2658-2668, 2010.

285. O'Brien SG, Guilhot F, Larson RA, Gathmann I, Baccarani M, Cervantes F, Cornelissen JJ, Fischer T, Hochhaus A, Hughes T, Lechner K, Nielsen JL, Rousselot P, Reiffers J, Saglio G, Shepherd J, Simonsson B, Gratwohl A, Goldman JM, Kantarjian H, Taylor K, Verhoef G, Bolton AE, Capdeville R, and Druker BJ; IRIS Investigators. Imatinib compared with interferon and lowdose cytarabine for newly diagnosed chronic-phase chronic myeloid leukemia. N Engl J Med 348: 994-1004, 2003.

286. Odiete O, Hill MF, and Sawyer DB. Neuregulin in cardiovascular development and disease. Circ Res 111: 1376-1385, 2012.

287. Oeffinger KC, Mertens AC, Sklar CA, Kawashima T, Hudson MM, Meadows AT, Friedman DL, Marina N, Hobbie W, Kadan-Lottick NS, Schwartz CL, Leisenring W, and Robison LL; Childhood Cancer Survivor Study. Chronic health conditions in adult survivors of childhood cancer. N Engl J Med 355: 1572-1582, 2006. 
288. Okazaki T, Tanaka Y, Nishio R, Mitsuiye T, Mizoguchi A, Wang J, Ishida M, Hiai H, Matsumori A, Minato N, and Honjo T. Autoantibodies against cardiac troponin I are responsible for dilated cardiomyopathy in PD-1deficient mice. Nat Med 9: 1477-1483, 2003.

289. Oliveira MS, Melo MB, Carvalho JL, Melo IM, Lavor MS, Gomes DA, de Goes AM, and Melo MM. Doxorubicin cardiotoxicity and cardiac function improvement after stem cell therapy diagnosed by strain echocardiography. J Cancer Sci Ther 5: 52-57, 2013.

290. Ong DS, Scherrer-Crosbie M, Coelho-Filho O, Francis SA, and Neilan TG. Imaging methods for detection of chemotherapy-associated cardiotoxicity and dysfunction. Expert Rev Cardiovasc Ther 12: 487-497, 2014.

291. Ozcelik C, Erdmann B, Pilz B, Wettschureck N, Britsch S, Hübner N, Chien KR, Birchmeier C, and Garratt AN. Conditional mutation of the ErbB2 (HER2) receptor in cardiomyocytes leads to dilated cardiomyopathy. Proc Natl Acad Sci U S A 99: 8880-8885, 2002.

292. Oztop I, Gencer M, Okan T, Yaren A, Altekin E, Turker $\mathrm{S}$, and Yilmaz U. Evaluation of cardiotoxicity of a combined bolus plus infusional 5-fluorouracil/folinic acid treatment by echocardiography, plasma troponin I level, QT interval and dispersion in patients with gastrointestinal system cancers. Jpn J Clin Oncol 34: 262268, 2004.

293. Pagliaro P, Moro F, Tullio F, Perrelli MG, and Penna C. Cardioprotective pathways during reperfusion: focus on redox signaling and other modalities of cell signaling. Antioxid Redox Signal 14: 833-850, 2011.

294. Pagliaro P and Penna C. Redox signalling and cardioprotection: translatability and mechanism. Br J Pharmacol 172: 1974-1995, 2015.

295. Papapetropoulos A, Foresti R, and Ferdinandy P. Pharmacology of the 'gasotransmitters' $\mathrm{NO}, \mathrm{CO}$ and $\mathrm{H} 2 \mathrm{~S}$ : translational opportunities. Br J Pharmacol 172: 13951396, 2015.

296. Pardoll DM. The blockade of immune checkpoints in cancer immunotherapy. Nat Rev Cancer 12: 252-264, 2012.

297. Paydas S. Dasatinib, large granular lymphocytosis, and pleural effusion: useful or adverse effect? Crit Rev Oncol Hematol 89: 242-247, 2014.

298. Penna C, Angotti C, and Pagliaro P. Protein Snitrosylation in preconditioning and postconditioning. Exp Biol Med (Maywood) 239: 647-662, 2014.

299. Pepe A, Pizzino F, Gargiulo P, Perrone-Filardi P, Cadeddu C, Mele D, Monte I, Novo G, Zito C, and Di Bella G. Cardiovascular imaging in the diagnosis and monitoring of cardiotoxicity: cardiovascular magnetic resonance and nuclear cardiology. J Cardiovasc Med (Hagerstown) 17: S45-S54, 2016.

300. Pereira GC, Pereira SP, Tavares LC, Carvalho FS, Magalhães-Novais S, Barbosa IA, Santos MS, Bjork J, Moreno AJ, Wallace KB, and Oliveira PJ. Cardiac cytochrome $\mathrm{c}$ and cardiolipin depletion during anthracyclineinduced chronic depression of mitochondrial function. Mitochondrion 30: 95-104, 2016.

301. Perik PJ, Lub-De Hooge MN, Gietema JA, van der Graaf WT, de Korte MA, Jonkman S, Kosterink JG, van Veldhuisen DJ, Sleijfer DT, Jager PL, and de Vries EG. Indium111-labeled trastuzumab scintigraphy in patients with human epidermal growth factor receptor 2-positive metastatic breast cancer. J Clin Oncol 24: 2276-2282, 2006.
302. Petrelli F, Barni S, Bertocchi P, and Zaniboni A. TAS102 , the first "cardio-gentle" fluoropyrimidine in the colorectal cancer landscape? BMC Cancer 16: 386, 2016.

303. Pick AM and Nystrom KK. Pazopanib for the treatment of metastatic renal cell carcinoma. Clin Ther 34: 511-520, 2012.

304. Pitt JM, Vetizou M, Daillere R, Roberti MP, Yamazaki T, Routy B, Lepage P, Boneca IG, Chamaillard M, Kroemer $\mathrm{G}$, and Zitvogel L. Resistance mechanisms to immunecheckpoint blockade in cancer: tumor-intrinsic and -extrinsic factors. Immunity 44: 1255-1269, 2016.

305. Pituskin E, Haykowsky M, Mackey JR, Thompson RB, Ezekowitz J, Koshman S, Oudit G, Chow K, Pagano JJ, and Paterson I. Rationale and design of the Multidisciplinary Approach to Novel Therapies in Cardiology Oncology Research Trial (MANTICORE 101-Breast): a randomized, placebo-controlled trial to determine if conventional heart failure pharmacotherapy can prevent trastuzumab-mediated left ventricular remodeling among patients with HER2+ early breast cancer using cardiac MRI. BMC Cancer 11: 318, 2011.

306. Pizzino F, Vizzari G, Qamar R, Bomzer C, Carerj S, Zito $\mathrm{C}$, and Khandheria BK. Multimodality imaging in cardiooncology. J Oncol 2015: 263950, 2015.

307. Plana JC, Galderisi M, Barac A, Ewer MS, Ky B, Scherrer-Crosbie M, Ganame J, Sebag IA, Agler DA, Badano LP, Banchs J, Cardinale D, Carver J, Cerqueira M, DeCara JM, Edvardsen T, Flamm SD, Force T, Griffin BP, Jerusalem G, Liu JE, Magalhães A, Marwick T, Sanchez LY, Sicari R, Villarraga HR, and Lancellotti P. Expert consensus for multimodality imaging evaluation of adult patients during and after cancer therapy: a report from the American Society of Echocardiography and the European Association of Cardiovascular Imaging. Eur Heart J Cardiovasc Imaging 15: 1063-1093, 2014.

308. Pointon AV, Walker TM, Phillips KM, Luo J, Riley J, Zhang SD, Parry JD, Lyon JJ, Marczylo EL, and Gant TW. Doxorubicin in vivo rapidly alters expression and translation of myocardial electron transport chain genes, leads to ATP loss and caspase 3 activation. PLoS One 5: e12733, 2010.

309. Polk A, Vaage-Nilsen M, Vistisen K, and Nielsen DL. Cardiotoxicity in cancer patients treated with 5fluorouracil or capecitabine: a systematic review of incidence, manifestations and predisposing factors. Cancer Treat Rev 39: 974-984, 2013.

310. Polk A, Vistisen K, Vaage-Nilsen M, and Nielsen DL. A systematic review of the pathophysiology of 5fluorouracil-induced cardiotoxicity. BMC Pharmacol Toxicol 15: 47, 2014.

311. Ponikowski P, Voors AA, Anker SD, Bueno H, Cleland JG, Coats AJ, Falk V, González-Juanatey JR, Harjola VP, Jankowska EA, Jessup M, Linde C, Nihoyannopoulos P, Parissis JT, Pieske B, Riley JP, Rosano GM, Ruilope LM, Ruschitzka F, Rutten FH, and van der Meer P; Authors/ Task Force Members; Document Reviewers. 2016 ESC Guidelines for the diagnosis and treatment of acute and chronic heart failure: the Task Force for the diagnosis and treatment of acute and chronic heart failure of the European Society of Cardiology (ESC). Developed with the special contribution of the Heart Failure Association (HFA) of the ESC. Eur J Heart Fail 18: 891-975, 2016.

312. Quintás-Cardama A, Kantarjian H, O'brien S, Borthakur G, Bruzzi J, Munden R, and Cortes J. Pleural effusion in 
patients with chronic myelogenous leukemia treated with dasatinib after imatinib failure. J Clin Oncol 25: 39083914, 2007.

313. Racil Z, Razga F, Drapalova J, Buresova L, Zackova D, Palackova M, Semerad L, Malaskova L, Haluzik M, and Mayer J. Mechanism of impaired glucose metabolism during nilotinib therapy in patients with chronic myelogenous leukemia. Haematologica 98: e124-e126, 2013.

314. Rainer PP, Doleschal B, Kirk JA, Sivakumaran V, Saad Z, Groschner K, Maechler H, Hoefler G, Bauernhofer T, Samonigg H, Hutterer G, Kass DA, Pieske B, von Lewinski D, and Pichler M. Sunitinib causes dose-dependent negative functional effects on myocardium and cardiomyocytes. BJU Int 110: 1455-1462, 2012.

315. Ranpura V, Hapani S, Chuang J, and Wu S. Risk of cardiac ischemia and arterial thromboembolic events with the angiogenesis inhibitor bevacizumab in cancer patients: a meta-analysis of randomized controlled trials. Acta Oncol 49: 287-297, 2010.

316. Rastogi S, Sharov VG, Mishra S, Gupta RC, Blackburn B, Belardinelli L, Stanley WC, and Sabbah HN. Ranolazine combined with enalapril or metoprolol prevents progressive LV dysfunction and remodeling in dogs with moderate heart failure. Am J Physiol Heart Circ Physiol 295: H2149-H2155, 2008.

317. Rea D, Mirault T, Cluzeau T, Gautier JF, Guilhot F, Dombret H, and Messas E. Early onset hypercholesterolemia induced by the 2nd-generation tyrosine kinase inhibitor nilotinib in patients with chronic phase-chronic myeloid leukemia. Haematologica 99: 1197-1203, 2014.

318. Reck M, Kaiser R, Mellemgaard A, Douillard JY, Orlov S, Krzakowski M, von Pawel J, Gottfried M, Bondarenko I, Liao M, Gann CN, Barrueco J, Gaschler-Markefski B, Novello S, and LUME-Lung 1 Study Group. Docetaxel plus nintedanib versus docetaxel plus placebo in patients with previously treated non-small-cell lung cancer (LUME-Lung 1): a phase 3, double-blind, randomised controlled trial. Lancet Oncol 15: 143-155, 2014.

319. Reus TL, Robert AW, Da Costa MB, de Aguiar AM, and Stimamiglio MA. Secretome from resident cardiac stromal cells stimulates proliferation, cardiomyogenesis and angiogenesis of progenitor cells. Int J Cardiol 221: 396403, 2016.

320. Riad A, Bien S, Westermann D, Becher PM, Loya K, Landmesser U, Kroemer HK, Schultheiss HP, and Tschöpe C. Pretreatment with statin attenuates the cardiotoxicity of Doxorubicin in mice. Cancer Res 69: 695699, 2009.

321. Richards CJ, Je Y, Schutz FA, Heng DY, Dallabrida SM, Moslehi JJ, and Choueiri TK. Incidence and risk of congestive heart failure in patients with renal and nonrenal cell carcinoma treated with sunitinib. J Clin Oncol 29: 3450-3456, 2011.

322. Rickard J, Kumbhani DJ, Baranowski B, Martin DO, Tang WH, and Wilkoff BL. Usefulness of cardiac resynchronization therapy in patients with Adriamycininduced cardiomyopathy. Am J Cardiol 105: 522-526, 2010.

323. Ridnour LA, Thomas DD, Donzelli S, Espey MG, Roberts DD, Wink DA, and Isenberg JS. The biphasic nature of nitric oxide responses in tumor biology. Antioxid Redox Signal 8: 1329-1337, 2006.

324. Rini BI, Escudier B, Tomczak P, Kaprin A, Szczylik C, Hutson TE, Michaelson MD, Gorbunova VA, Gore ME,
Rusakov IG, Negrier S, Ou YC, Castellano D, Lim HY, Uemura H, Tarazi J, Cella D, Chen C, Rosbrook B, Kim $\mathrm{S}$, and Motzer RJ. Comparative effectiveness of axitinib versus sorafenib in advanced renal cell carcinoma (axis): a randomised phase 3 trial. Lancet 378: 1931-1939, 2011.

325. Rohrbach S, Niemann B, Silber RE, and Holtz J. Neuregulin receptors erbB2 and erbB4 in failing human myocardium-depressed expression and attenuated activation. Basic Res Cardiol 100: 240-249, 2005.

326. Rohrbach S, Yan X, Weinberg EO, Hasan F, Bartunek J, Marchionni MA, and Lorell BH. Neuregulin in cardiac hypertrophy in rats with aortic stenosis. Differential expression of erbB2 and erbB4 receptors. Circulation 100: 407-412, 1999.

327. Romano S, Fratini S, Procaccini V, Stifano G, Mancini M, Di Mauro M, Ficorella C, and Penco M. Serial measurements of NT-proBNP are predictive of not-high-dose anthracycline cardiotoxicity in breast cancer patients. $\mathrm{Br} \mathrm{J}$ Cancer 105: 1663-1668, 2011.

328. Rosenthal A, Luthi J, Belohlavek M, Kortüm KM, Mookadam F, Mayo A, Fonseca R, Bergsagel PL, Reeder CB, Mikhael JR, and Stewart AK. Carfilzomib and the cardiorenal system in myeloma: an endothelial effect? Blood Cancer J 6: e384, 2016.

329. Rosti G, Martinelli G, and Baccarani M. In reply to 'Cardiotoxicity of the cancer therapeutic agent imatinib mesylate'. Nat Med 13: 15, 2007.

330. Rowinsky EK, Eisenhauer EA, Chaudhry V, Arbuck SG, and Donehower RC. Clinical toxicities encountered with paclitaxel (Taxol). Semin Oncol 20: 1-15, 1993.

331. Russell SD, Lyon A, Lenihan DJ, Moreau P, Joshua D, ChngW-J, Palumbo A, Goldschmidt H, Hájek R, Facon T, Ludwig H, Pour L, Niesvizky R, Oriol A, Rosinol L, Suvorov A, Gaidano G, Goranova-Marinova V, Gillenwater HH, Mohamed N, Feng S, and Dimopoulos MA. Serial echocardiographic assessment of patients (pts) with relapsed multiple myeloma (RMM) receiving carfilzomib and dexamethasone (Kd) vs bortezomib and dexamethasone (Vd): a substudy of the phase 3 Endeavor Trial (NCT01568866). Blood 126: 4250, 2015.

332. Salesse $S$ and Verfaillie CM. BCR/ABL: from molecular mechanisms of leukemia induction to treatment of chronic myelogenous leukemia. Oncogene 21: 8547-8559, 2002.

333. Salvatorelli E, Menna $P$, and Minotti G. Managing anthracycline-induced cardiotoxicity: beginning with the end in mind. Future Cardiol 11: 363-366, 2015.

334. Sandler A, Gray R, Perry MC, Brahmer J, Schiller JH, Dowlati A, Lilenbaum R, and Johnson DH. Paclitaxelcarboplatin alone or with bevacizumab for nonsmall-cell lung cancer. N Engl J Med 355: 2542-2550, 2006.

335. Sandri MT, Salvatici M, Cardinale D, Zorzino L, Passerini R, Lentati P, Leon M, Civelli M, Martinelli G, and Cipolla CM. N-terminal pro-B-type natriuretic peptide after highdose chemotherapy: a marker predictive of cardiac dysfunction? Clin Chem 51: 1405-1410, 2005.

336. Sano M, Minamino T, Toko H, Miyauchi H, Orimo M, Qin Y, Akazawa H, Tateno K, Kayama Y, Harada M, Shimizu I, Asahara T, Hamada H, Tomita S, Molkentin JD, Zou Y, and Komuro I. p53-induced inhibition of HIF1 causes cardiac dysfunction during pressure overload. Nature 446: 444-448, 2007.

337. Santos DL, Moreno AJM, Leino RL, Froberg MK, and Wallace KB. Carvedilol protects against doxorubicin- 
induced mitochondrial cardiomyopathy Toxicol Appl Pharmacol 185: 218-227, 2002.

338. Sanz-Ruiz R, Bolli R, Gersh BJ, Janssens S, Menasché P, Perin EC, Taylor DA, Terzic A, Willerson JT, and Fernández-Avilés. The TACTIS initiative: time for a global alliance on cardiovascular regenerative medicine. Eur Heart J 37: 2208-2211, 2016.

339. Sasaki K, Strom SS, O’Brien S, Jabbour E, Ravandi F, Konopleva M, Borthakur G, Pemmaraju N, Daver N, Jain P, Pierce S, Kantarjian H, and Cortes JE. Relative survival in patients with chronic-phase chronic myeloid leukaemia in the tyrosine-kinase inhibitor era: analysis of patient data from six prospective clinical trials. Lancet Haematol 5: e186-e193, 2015.

340. Sawaya H, Sebag IA, Plana JC, Januzzi JL, Ky B, Tan TC, Cohen V, Banchs J, Carver JR, Wiegers SE, Martin RP, Picard MH, Gerszten RE, Halpern EF, Passeri J, Kuter I, and Scherrer-Crosbie M. Assessment of echocardiography and biomarkers for the estende prediction of cardiotoxicity in patients treated with anthracyclines, taxanes, and trastuzumab. Circ Cardiovasc Imaging 5: 596-603, 2012.

341. Sawyer DB. Anthracyclines and heart failure. $N$ Engl J Med 368: 1154-1156, 2013.

342. Scartozzi M, Galizia E, Chiorrini S, Giampieri R, Berardi $\mathrm{R}$, Pierantoni $\mathrm{C}$, and Cascinu S. Arterial hypertension correlates with clinical outcome in colorectal cancer patients treated with first-line bevacizumab. Ann Oncol 20: 227-230, 2009.

343. Schermuly RT, Dony E, Ghofrani HA, Pullamsetti S, Savai R, Roth M, Sydykov A, Lai YJ, Weissmann N, Seeger W, and Grimminger F. Reversal of experimental pulmonary hypertension by PDGF inhibition. J Clin Invest 115: 2811-2821, 2005.

344. Schmidinger M, Zielinski CC, Vogl UM, Bojic A, Bojic M, Schukro C, Ruhsam M, Hejna M, and Schmidinger H. Cardiac toxicity of sunitinib and sorafenib in patients with metastatic renal cell carcinoma. J Clin Oncol 26: 5204 5212, 2008.

345. Scott JM, Khakoo A, Mackey JR, Haykowsky MJ, Douglas PS, and Jones LW. Modulation of anthracyclineinduced cardiotoxicity by aerobic exercise in breast cancer: current evidence and underlying mechanisms. Circulation 124: 642-650, 2011.

346. Scott JM, Koelwyn GJ, Hornsby WE, Khouri M, Peppercorn J, Douglas PS, and Jones LW. Exercise therapy as treatment for cardiovascular and oncologic disease after a diagnosis of early-stage cancer. Semin Oncol 40: 218-228, 2013.

347. Seicean S, Seicean A, Alan N, Plana JC, Budd GT, and Marwick TH. Cardioprotective effect of $\beta$-adrenoceptor blockade in patients with breast cancer undergoing chemotherapy: follow-up study of heart failure. Circ Heart Fail 6: 420-426, 2013.

348. Seicean S, Seicean A, Plana JC, Budd GT, and Marwick $\mathrm{TH}$. Effect of statin therapy on the risk for incident heart failure in patients with breast cancer receiving anthracycline chemotherapy: an observational clinical cohort study. J Am Coll Cardiol 60: 2384-2390, 2012.

349. Seidman A, Hudis C, Pierri MK, Shak S, Paton V, Ashby M, Murphy M, Stewart SJ, and Keefe D. Cardiac dysfunction in the trastuzumab clinical trials experience. $J$ Clin Oncol 20: 1215-1221, 2002.

350. Seymour L, Bramwell V, and Moran LA. Use of dexrazoxane as a cardioprotectant in patients receiving doxo- rubicin or epirubicin chemotherapy for the treatment of cancer. The Provincial Systemic Treatment Disease Site Group. Cancer Prev Control 3: 145-159, 1999.

351. Shah NP, Rousselot P, Schiffer C, Rea D, Cortes JE, Milone J, Mohamed H, Healey D, Kantarjian H, Hochhaus A, and Saglio G. Dasatinib in imatinib-resistant or -intolerant chronic-phase, chronic myeloid leukemia patients: 7-year follow-up of study CA180-034. Am J Hematol 91: 869-874, 2016.

352. Shah NP, Wallis N, Farber HW, Mauro MJ, Wolf RA, Mattei D, Guha M, Rea D, and Peacock A. Clinical features of pulmonary arterial hypertension in patients receiving dasatinib. Am J Hematol 90: 1060-1064, 2015.

353. Shalkey-Hahn V, Lenihan DJ, and Ky B. Cancer therapyinduced cardiotoxicity: basic mechanisms and potential cardioprotective therapies. J Am Heart Assoc 3: e000665, 2014.

354. Sharma P and Allison JP. Immune checkpoint targeting in cancer therapy: toward combination strategies with curative potential. Cell 161: 205-214, 2015.

355. Sharma $P$ and Allison JP. The future of immune checkpoint therapy. Science 348: 56-61, 2015.

356. Shimazaki C, Ochiai N, Uchida R, Fuchida SI, Okano A, Ashihara E, Inaba $\mathrm{T}$, Fujita $\mathrm{N}$, and Nakagawa M. Intramuscular edema as a complication of treatment with imatinib. Leukemia 17: 804-805, 2003.

357. Shoemaker LK, Arora U, Rocha, and Lima CM. 5fluorouracil-induced coronary vasospasm. Cancer Control 11: 46-49, 2004.

358. Simůnek T, Stérba M, Popelová O, Adamcová M, Hrdina $\mathrm{R}$, and Gersl V. Anthracycline-induced cardiotoxicity: overview of studies examining the roles of oxidative stress and free cellular iron. Pharmacol Rep 61: 154-171, 2009.

359. Singh CK, Siddigui IA, El-Abd S, Mukhtar H, and Ahmad N. Combination chemoprevention with grape antioxidants. Mol Nutr Food Res 60: 1406-1415, 2016.

360. Singla DK and Abdelli LS. Embryonic stem cells and released factors stimulate c-kit/FLK-1 progenitor cells and promote neovascularization in doxorubicin-induced cardiomyopathy. Cell Transplant 24: 1043-1052, 2015.

361. Slamon DJ, Clark GM, Wong SG, Levin WJ, Ullrich A, and McGuire WL. Human breast cancer: correlation of relapse and survival with amplification of the HER-2/neu oncogene. Science 235: 177-182, 1987.

362. Slamon DJ, Leyland-Jones B, Shak S, Fuchs H, Paton V, Bajamonde A, Fleming T, Eiermann W, Wolter J, Pegram $\mathrm{M}$, Baselga $\mathrm{J}$, and Norton L. Use of chemotherapy plus a monoclonal antibody against her2 for metastatic breast cancer that overexpresses HER2. N Engl J Med 344: 783792, 2001.

363. Smith RR, Barile L, Cho HC, Leppo MK, Hare JM, Messina E, Giacomello A, Abraham MR, and Marban E. Regenerative potential of cardiosphere-derived cells expanded from percutaneous endomyocardial biopsy specimens. Circulation 115: 896-908, 2007.

364. Soga M, Kamal FA, Watanabe K, Ma M, Palaniyandi S, Prakash P, Veeraveedu P, Mito S, Kunisaki M, Tachikawa $\mathrm{H}$, Kodama M, and Aizawa Y. Effects of angiotensin II receptor blocker (candesartan) in daunorubicin-induced cardiomyopathic rats. Int J Cardiol 110: 378-385, 2006.

365. Sorrentino MF, Kim J, Foderaro AE, and Truesdell AG. 5fluorouracil induced cardiotoxicity: review of the literature. Cardiol J 19: 453-458, 2012.

366. Spallarossa P, Garibaldi S, Altieri P, Fabbi P, Manca V, Nasti S, Rossettin P, Ghigliotti G, Ballestrero A, Patrone F, 
Barsotti A, and Brunelli C. Carvedilol prevents doxorubicininduced free radical release and apoptosis in cardiomyocytes in vitro. J Mol Cell Cardiol 37: 837-846, 2004.

367. Spallarossa P, Maurea N, Cadeddu C, Madonna R, Mele D, Monte I, Novo G, Pagliaro P, Pepe A, Tocchetti CG, Zito C, and Mercuro G. A recommended practical approach to the management of anthracycline-based chemotherapy cardiotoxicity: an opinion paper of the working group on drug cardiotoxicity and cardioprotection, Italian Society of Cardiology. J Cardiovasc Med (Hagerstown) 17: S84-S92, 2016.

368. Spur EM, Althof N, Respondek D, Klingel K, Heuser A, Overkleeft HS, and Voigt A. Inhibition of chymotrypticlike standard proteasome activity exacerbates doxorubicininduced cytotoxicity in primary cardiomyocytes. Toxicology 353-354: 34-47, 2016.

369. Stefani L, Maffulli N, Mascherini G, Francini L, Petri C, and Galanti G. Exercise as prescription therapy: benefits in cancer and hypertensive patients. Transl Med UniSa 11: 39-43, 2014.

370. Steingart RM, Bakris GL, Chen HX, Chen MH, Force T, Ivy SP, Leier CV, Liu G, Lenihan D, Lindenfeld J, Maitland ML, Remick SC, and Tang WH. Management of cardiac toxicity in patients receiving vascular endothelial growth factor signaling pathway inhibitors. Am Heart $J$ 163: 156-163, 2012.

371. Stejskal D, Lacnak B, Jedelsky L, Stepanova L, Proskova J, Solichova P, Kadalova L, Janosova M, Seitlova P, Karpisek M, and Sprongl L. Use of glycogen phosphorylase BB measurement with POCT in the diagnosis of acute coronary syndromes. A comparison with the ELISA method. Biomed Pap Med Fac Univ Palacky Olomouc Czech Repub 151: 247-249, 2007.

372. Stěrba M, Popelová O, Vávrová A, Jirkovský E, Kovaříková P, Geršl V, and Simůnek T. Oxidative stress, redox signaling, and metal chelation in anthracycline cardiotoxicity and pharmacological cardioprotection. Antioxid Redox Signal 18: 899-929, 2013.

373. Stewart AK, Rajkumar SV, Dimopoulos MA, Masszi TS, Špička I, Albert Oriol, Hajek R, Rosinol L, Siegel DS, Mihaylov GG, Goranova-Marinova V, Rajnics P, Suvorov A, Ruben Niesvizky R, Jakubowiak AJ, San-Miguel JF, Ludwig H, Wang M, Maisnar V, Minarik J, Bensinger WI, Mateos MV, Ben-Yehuda D, Kukreti V, Zojwalla N, Tonda ME, Yang X, Xing B, Moreau P, and Palumbo A. Carfilzomib, lenalidomide, and dexamethasone for relapsed multiple myeloma. N Engl J Med 372: 142-152, 2015.

374. Su EJ, Fredriksson L, Geyer M, Folestad E, Cale J, Andrae J, Gao Y, Pietras K, Mann K, Yepes M, Strickland DK, Betsholtz C, Eriksson U, and Lawrence DA. Activation of PDGF-CC by tissue plasminogen activator impairs blood-brain barrier integrity during ischemic stroke. Nat Med 14: 731-773, 2008.

375. Sultana N, Zhang L, Yan J, Chen J, Cai W, Razzague S, Jeong D, Sheng W, Bu L, Xu M, Huang GY, Hajar RJ, Zhou B, Moon A, and Cai CL. Resident c-kit $(+)$ cells in the heart are not cardiac stem cells. Nat Commun 6: 8701, 2015.

376. Suter TM and Ewer MS. Cancer drugs and the heart: importance and management. Eur Heart J 34: 1102-1111, 2013.

377. Suter TM, Procter M, van Veldhuisen DJ, Muscholl M, Bergh J, Carlomagno C, Perren T, Passalacqua R, Bighin
C, Klijn JG, Ageev FT, Hitre E, Groetz J, Iwata H, Knap M, Gnant M, Muehlbauer S, Spence A, Gelber RD, and Piccart-Gebhart MJ. Trastuzumab-associated cardiac adverse effects in the herceptin adjuvant trial. J Clin Oncol 25: 3859-3865, 2007.

378. Suzuki T, Hayashi D, Yamazaki T, Mizuno T, Kanda Y, Komuro I, Kurabayashi M, Yamaoki K, Mitani K, Hirai $\mathrm{H}$, Nagai R, and Yazaki Y. Elevated B-type natriuretic peptide levels after anthracycline administration. Am Heart J 136: 362-363, 1998.

379. Swain SM and Vici P. The current and future role of dexrazoxane as a cardioprotectant in anthracycline treatment: expert panel review. J Cancer Res Clin Oncol 130: $1-7,2004$.

380. Swain SM, Whaley FS, and Ewer MS. Congestive heart failure in patients treated with doxorubicin: a retrospective analysis of three trials. Cancer 97: 2869-2879, 2003.

381. Swain SM, Whaley FS, Gerber MC, Weisberg S, York M, Spicer D, Jones SE, Wadler S, Desai A, Vogel C, Speyer J, Mittelman A, Reddy S, Pendergrass K, Velez-Garcia E, Ewer MS, Bianchine JR, and Gams RA. Cardioprotection with dexrazoxane for doxorubicin-containing therapy in advanced breast cancer. J Clin Oncol 15: 1318-1332, 1997.

382. Sysa-Shah P, Tocchetti CG, Gupta M, Rainer PP, Shen X, Kang BH, Belmonte F, Li J, Xu Y, Guo X, Bedja D, Gao WD, Paolocci N, Rath R, Sawyer DB, Naga Prasad SV, and Gabrielson K. Bidirectional cross-regulation between ErbB2 and $\beta$-adrenergic signalling pathways. Cardiovasc Res 109: 358-373, 2016.

383. Talpaz M, Shah NP, Kantarjian H, Donato N, Nicoll J, Paquette R, Cortes J, O’Brien S, Nicaise C, Bleickardt E, Blackwood-Chirchir MA, Iyer V, Chen TT, Huang F, Decillis AP, and Sawyers CL. Dasatinib in imatinibresistant Philadelphia chromosome-positive leukemias. $N$ Engl J Med 354: 2531-2541, 2006.

384. Tan C, Tasaka H, Yu KP, Murphy ML, and Karnofsky DA. Daunomycin, an antitumor antibiotic, in the treatment of neoplastic disease. Clinical evaluation with special reference to childhood leukemia. Cancer 20: 333-353, 1967.

385. Tarrio ML, Grabie N, Bu DX, Sharpe AH, and Lichtman AH. PD-1 protects against inflammation and myocyte damage in T cell-mediated myocarditis. J Immunol 188: 4876-4884, 2012.

386. Tassan-Mangina S, Codorean D, Metivier M, Costa B, Himberlin C, Jouannaud C, Blaise AM, Elaerts J, and Nazeyrollas P. Tissue Doppler imaging and conventional echocardiography after anthracycline treatment in adults: early and late alterations of left ventricular function during a prespective study. Eur J Echocardiogr 7: 141-146, 2006.

387. Telli ML, Witteles RM, Fisher GA, and Srinivas S. Cardiotoxicity associated with the cancer therapeutic agent sunitinib malate. Ann Oncol 19: 1613-1618, 2008.

388. Thavendiranathan $\mathrm{P}$, Wintersperger BJ, Flamm SD, and Marwick TH. Cardiac MRI in the assessment of cardiac injury and toxicity from cancer chemotherapy: a systematic review. Circ Cardiovasc Imaging 6: 1080-1091, 2013.

389. Thorn CF, Oshiro C, Marsh S, Hernandez-Boussard T, McLeod H, Klein TE, and Altman RB. Doxorubicin pathways: pharmacodynamics and adverse effects. Pharmacogenet Genomics 21: 440-446, 2011. 
390. Timolati F, Ott D, Pentassuglia L, Giraud MN, Perriard JC, Suter TM, and Zuppinger C. Neuregulin-1 beta attenuates doxorubicin-induced alterations of excitationcontraction coupling and reduces oxidative stress in adult rat cardiomyocytes. J Mol Cell Cardiol 41: 845-854, 2006.

391. Tocchetti CG, Carpi A, Coppola C, Quintavalle C, Rea D, Campesan M, Arcari A, Piscopo G, Cipresso C, Monti MG, De Lorenzo C, Arra C, Condorelli G, Di Lisa F, and Maurea N. Ranolazine protects from doxorubicin-induced oxidative stress and cardiac dysfunction. Eur J Heart Fail 16: 358-366, 2014.

392. Tocchetti CG, Gallucci G, Coppola C, Piscopo G, Cipresso C, Maurea C, Giudice A, Iaffaioli RV, Arra C, and Maurea N. The emerging issue of cardiac dysfunction induced by antineoplastic angiogenesis inhibitors. Eur $J$ Heart Fail 15: 482-489, 2013.

393. Tocchetti CG, Leppo M, Bedja D, Wang Y, Weiss RG and Paolocci N. Cardiac overexpression of creatine kinase improves cardiomyocytes function in heart failure and during increased redox stress. Circ Res 117: A338, 2015.

394. Tocchetti CG, Molinaro M, Angelone T, Lionetti V, Madonna R, Mangiacapra F, Moccia F, Penna C, Sartiani L, Quaini F, and Pagliaro P. Nitroso-Redox Balance and modulation of basal myocardial function: an update from the Italian Society of Cardiovascular Research (SIRC). Curr Drug Targets 16: 895-903, 2015.

395. Todaro MC, Khandheria BK, Longobardo L, Zito C, Cusmà-Piccione M, Di Bella G, Oreto L, Mohammed M, Oreto $\mathrm{G}$, and Carerj S. New diagnostic perspectives on heart failure with preserved ejection fraction: systolic function beyond ejection fraction. J Cardiovasc Med (Hagerstown) 16: 527-537, 2015.

396. Topalian SL, Drake CG, and Pardoll DM. Immune checkpoint blockade: a common denominator approach to cancer therapy. Cancer Cell 27: 450-461, 2015.

397. Topalian SL, Hodi FS, Brahmer JR, Gettinger SN, Smith DC, McDermott DF, Powderly JD, Carvajal RD, Sosman JA, Atkins MB, Leming PD, Spigel DR, Antonia SJ, Horn L, Drake CG, Pardoll DM, Chen L, Sharfman WH, Anders RA, Taube JM, McMiller TL, Xu H, Korman AJ, JureKunkel M, Agrawal S, McDonald D, Kollia GD, Gupta A, Wigginton JM, and Sznol M. Safety, activity, and immune correlates of anti-PD-1 antibody in cancer. $N$ Engl $\mathrm{J}$ Med 366: 2443-2454, 2012.

398. Tsibiribi P, Bui-Xuan C, Bui-Xuan B, Lombard-Bohas C, Duperret S, Belkhiria M, and Tomour Q. Cardiac lesions induced by 5-fluorouracil in the rabbit. Hum Exp Toxicol 25: 305-309, 2006.

399. Turnis ME, Andrews LP, and Vignali DA. Inhibitory receptors as targets for cancer immunotherapy. Eur J Immunol 45: 1892-1905, 2015.

400. Uray IP, Connelly JH, Thomázy V, Shipley GL, Vaughn WK, Frazier OH, Taegtmeyer H, and Davies PJ. Left ventricular unloading alters receptor tyrosine kinase expression in the failing human heart. $J$ Heart Lung Transplant 21: 771-782, 2002.

401. Valdés Olmos RA, Carrió I, Hoefnagel CA, Estorch M, ten Bokkel Huinink WW, López-Pousa J, and Dalesio O. High sensitivity of radiolabelled antimyosin scintigraphy in assessing anthracycline related early myocyte damage preceding cardiac dysfunction. Nucl Med Commun 23: $871-877,2002$.
402. Valdés Olmos RA, ten Bokkel Huinink WW, Dewit LG, Hoefnagel CA, Liem IH, and van Tinteren H. Iodine-123 metaiodobenzylguanidine in the assessment of late cardiac effects from cancer therapy. Eur J Nucl Med 23: 453-458, 1996.

403. Valent P. Severe adverse events associated with the use of second-line BCR/ABL tyrosine kinase inhibitors: preferential occurrence in patients with comorbidities. Haematologica 96: 1395-1397, 2011.

404. Van Berlo JH, Kanisicak O, Maillet M, Vagnozzi RJ, Karch J, Lin SC, Middleton RC, Marbán E, and Molkentin JD. c-kit+ cells minimally contribute cardiomyocytes to the heart. Nature 509: 337-341, 2014.

405. Van Berlo JH and Molkentin JD. An emerging consensus on cardiac regeneration. Nat Med 20: 1386-1393, 2014.

406. Van Berlo JH and Molkentin JD. Most of the dust has settled: cKit+ progenitor cells are an irrelevant source of cardiac myocytes in vivo. Circ Res 118: 17-19, 2016.

407. Van Cutsem E, Bajetta E, Valle J, Köhne CH, Hecht JR, Moore M, Germond C, Berg W, Chen BL, Jalava T, Lebwohl D, Meinhardt G, Laurent D, and Lin E. Randomized, placebo-controlled, phase III study of oxaliplatin, fluorouracil, and leucovorin with or without PTK787/ZK 222584 in patients with previously treated metastatic colorectal adenocarcinoma. J Clin Oncol 29: 2004-2010, 2011.

408. Van Cutsem E, Hoff PM, Blum JL, Abt M, and Osterwalder B. Incidence of cardiotoxicity with the oral fluoropyrimidine capecitabine is typical of that reported with 5-fluorouracil. Ann Oncol 13: 484-485, 2002.

409. Varga ZV, Ferdinandy P, Liaudet L, and Pacher P. Druginduced mitochondrial dysfunction and cardiotoxicity. Am J Physiol Heart Circ Physiol 30: H1453-H1467, 2015.

410. Vejpongsa P and Yeh ET. Prevention of anthracyclineinduced cardiotoxicity: challenges and opportunities. $\mathrm{J} \mathrm{Am}$ Coll Cardiol 64: 938-945, 2014.

411. Vermorken JB, Remenar E, van Herpen C, Gorlia T, Mesia R, Degardin M, Stewart JS, Jelic S, Betka J, Preiss JH, van den Weyngaert D, Awada A, Cupissol D, Kienzer HR, Rey A, Desaunois I, Bernier J, and Lefebvre JL; EORTC 24971/TAX 323 Study Group. Cisplatin, fluorouracil, and docetaxel in unresectable head and neck cancer. N Eng J Med 357: 1695-1704, 2007.

412. Vincent DT, Ibrahim YF, Espey MG, and Suzuki YJ. The role of antioxidants in the era of cardio-oncology. Cancer Chemother Pharmacol 72: 1157-1168, 2013.

413. Vlasova II, Tyurin VA, Kapralov AA, Kurnikov IV, Osipov AN, Potapovich MV, Stoyanovsky DA, and Kagan VE. Nitric oxide inhibits peroxidase activity of cytochrome c.cardiolipin complex and blocks cardiolipin oxidation. J Biol Chem 281: 14554-14562, 2006.

414. Voskens CJ1, Goldinger SM, Loquai C, Robert C, Kaehler KC, Berking C, Bergmann T, Bockmeyer CL, Eigentler T, Fluck M, Garbe C, Gutzmer R, Grabbe S, Hauschild A, Hein R, Hundorfean G, Justich A, Keller U, Klein C, Mateus C, Mohr P, Paetzold S, Satzger I, Schadendorf D, Schlaeppi M, Schuler G, Schuler-Thurner B, Trefzer U, Ulrich J, Vaubel J, von Moos R, Weder P, Wilhelm T, Göppner D, Dummer R, and Heinzerling LM. The price of tumor control: an analysis of rare side effects of antiCTLA-4 therapy in metastatic melanoma from the ipilimumab network. PLoS One 8: e53745, 2013.

415. Wakasugi S1, Wada A, Hasegawa Y, Nakano S, and Shibata N. Detection of abnormal cardiac adrenergic 
neuron activity in adriamycin-induced cardiomyopathy with iodine-125-metaiodobenzylguanidine. J Nucl Med 33: 208-214, 1992.

416. Walker J, Bhullar N, Fallah-Rad N, Lytwyn M, Golian M, Fang T, Summers AR, Singal PK, Barac I, Kirkpatrick ID, and Jassal DS. Role of three-dimensional echocardiography in breast cancer: comparison with two-dimensional echocardiography, multiple-gated acquisition scans, and cardiac magnetic resonance imaging. J Clin Oncol 28: 3429-3436, 2010.

417. Walsh K and Shiojima I. Cardiac growth and angiogenesis coordinated by intertissue interactions. J Clin Invest 117 : 3176-3179, 2007.

418. Wang J, Okazaki IM, Yoshida T, Chikuma S, Kato Y, Nakaki F, Hiai H, Honjo T, and Okazaki T. PD-1 deficiency results in the development of fatal myocarditis in MRL mice. Int Immunol 22: 443-452, 2010.

419. Wang X, Ha T, Liu L, Zou J, Zhang X, Kalbfleisch J, Gao $\mathrm{X}$, Williams $\mathrm{D}$, and $\mathrm{Li} \mathrm{C}$. Increased expression of microRNA146a decreases myocardial ischaemia/reperfusion injury. Cardiovasc Res 97: 432-442, 2013.

420. Weber JS, Kahler KC, and Hauschild A. Management of immune-related adverse events and kinetics of response with ipilimumab. J Clin Oncol 30: 2691-2697, 2012.

421. Welti J, Loges S, Dimmeler S, and Carmeliet P. Recent molecular discoveries in angiogenesis and antiangiogenic therapies in cancer. J Clin Invest 123: 3190-3200, 2013.

422. Will Y, Dykens JA, Nadanaciva S, Hirakawa B, Jamieson J, Marroguin LD, Hynes J, Patyna S, and Jessen BA. Effect of the multitargeted tyrosine kinase inhibitors imatinib, dasatinib, sunitinib and sorefenib on mitochondrial function in isolated rat heart mitochondria and $\mathrm{H} 9 \mathrm{c} 2$ cells. Toxicol Sci 106: 153-161, 2008.

423. Willis MS and Patterson C. Into the heart: the emerging role of the ubiquitin-proteasome system. J Mol Cell Cardiol 41: 567-579, 2006.

424. Willis MS and Patterson C. Proteotoxicity and cardiac dysfunction-Alzheimer's disease of the heart? N Engl J Med 368: 455-464, 2013.

425. Wouters KA, Kremer LC, Miller TL, Herman EH, and Lipshultz SE. Protecting against anthracycline-induced myocardial damage: a review of the most promising strategies. Br J Haematol 131: 561-578, 2005.

426. Xi L, Zhu SG, Das A, Chen Q, Durrant D, Hobbs DC, Lesnefsky EJ, and Kukreja RC. Dietary inorganic nitrate alleviates doxorubicin cardiotoxicity: mechanisms and implications. Nitric Oxide 26: 274-284, 2012.

427. Xiao Y, Yin J, Wei J, and Shang Z. Incidence and risk of cardiotoxicity associated with bortezomib in the treatment of cancer: a systematic review and meta-analysis. PLoS One 9: e87671, 2014.

428. Yamagishi S and Takeuchi M. Telmisartan is a promising cardiometabolic sartan due to its unique PPAR-gammainducing property. Med Hypotheses 64: 476-478, 2005.

429. Yeh ET and Bickford CL. Cardiovascular complications of cancer therapy: incidence, pathogenesis, diagnosis, and management. J Am Coll Cardiol 53: 2231-2247, 2009.

430. Yue TL, Cheng HY, Lysko PG, McKenna PJ, Feuerstein R, Gu JL, Lysko KA, Davis LL, and Feuerstein G. Carvedilol, a new vasodilator and beta adrenoceptor antagonist, is an antioxidant and free radical scavenger. $J$ Pharmacol Exp Ther 263: 92-98, 1992.
431. Yun S, Vincelette ND, Mansour I, Hariri D, and Motamed S. Late onset ipilimumab-induced pericarditis and pericardial effusion: a rare but life threatening complication. Case Rep Oncol Med 2015: 794842, 2015.

432. Zamorano JL, Lancellotti P, Rodriguez Muñoz D, Aboyans V, Asteggiano R, Galderisi M, Habib G, Lenihan DJ, Lip GY, Lyon AR, Lopez Fernandez T, Mohty D, Piepoli MF, Tamargo J, Torbicki A, and Suter TM; Authors/Task Force Members; ESC Committee for Practice Guidelines (CPG). 2016 ESC Position Paper on cancer treatments and cardiovascular toxicity developed under the auspices of the ESC Committee for Practice Guidelines: The Task Force for cancer treatments andcardiovascular toxicity of the European Society of Cardiology (ESC). Eur Heart J 37: 2768-2801, 2016.

433. Zanardelli M, Micheli L, Cinci L, Failli P, Ghelardini C, and Di Cesare Mannelli L. Oxalipla: 2tin neurotoxicity involves peroxisome alterations. PPAR $\gamma$ agonism as preventive pharmacological approach. PLoS One 9: e102758, 2014.

434. Zang J, Wu S, Tang L, Xu X, Bai J, Ding C, Chang Y, Yue L, Kang E, and He J. Incidence and risk of QTc interval prolongation among cancer patients treated with vandetanib: a systematic review and meta-analysis. PLoS One 7: e30353Z, 2012.

435. Zhang S, Liu X, Bawa-Khalfe T, Lu LS, Lyu YL, Liu LF, and Yeh ET. Identification of the molecular basis of doxorubicin-induced cardiotoxicity. Nat Med 18: 16391642, 2012.

436. Zhang Y, Sivakumaran P, Newcomb AE, Hernandez D, Harris N, Khana bdali R, Liu GS, Kelly DJ, Pébay A, Hewitt AW, Boyle A, Harvey R, Morrison WA, Elliot DA, Dusting GJ, and Lim SY. Cardiac repair with a novel population of mesenchymal stem cells resident in the human heart. Stem Cells 33: 3100-3113, 2015.

437. Zhu W, Zou Y, Aikawa R, Harada K, Kudoh S, Uozumi H, Hayashi D, Gu Y, Yamazaki T, Nagai R, Yazaki Y, and Komuro I. MAPK superfamily plays an important role in daunomycin-induced apoptosis of cardiac myocytes. Circulation 100: 2100-2107, 1999.

438. Zito C, Longobardo L, Cadeddu C, Monte I, Novo G, Dell'Oglio S, Pepe A, Madonna R, Tocchetti CG, and Mele D. Cardiovascular imaging in the diagnosis and monitoring of cardiotoxicity: role of echocardiography. $J$ Cardiovasc Med (Hagerstown) 17: S35-S44, 2016.

Address correspondence to: Dr. Pasquale Pagliaro Department of Clinical and Biological Sciences University of Turin Regione Gonzole 10 10043 Turin Italy

E-mail: pasquale.pagliaro@unito.it

Date of first submission to ARS Central, October 26, 2016; date of final revised submission, April 6, 2017; date of acceptance, April 10, 2017. 


\begin{tabular}{|c|}
\hline 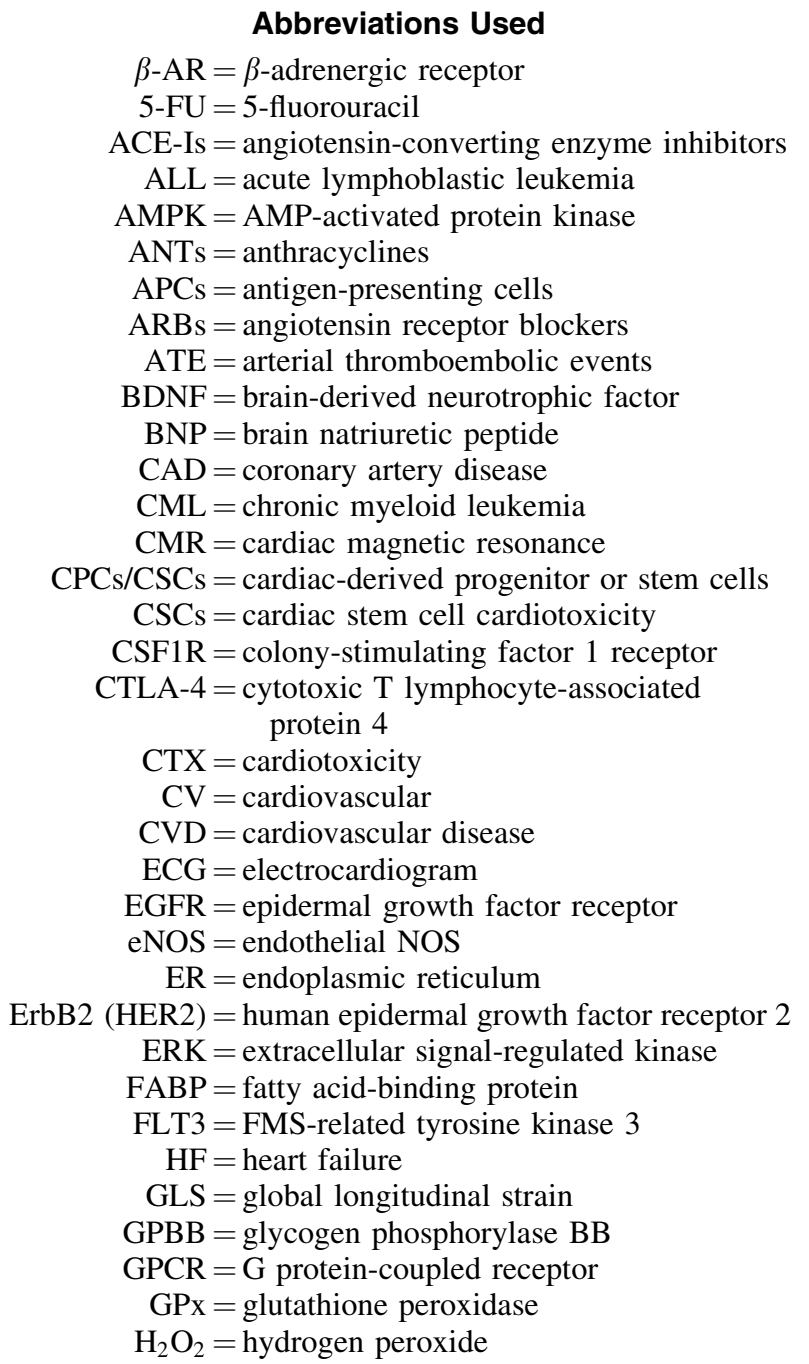 \\
\hline
\end{tabular}

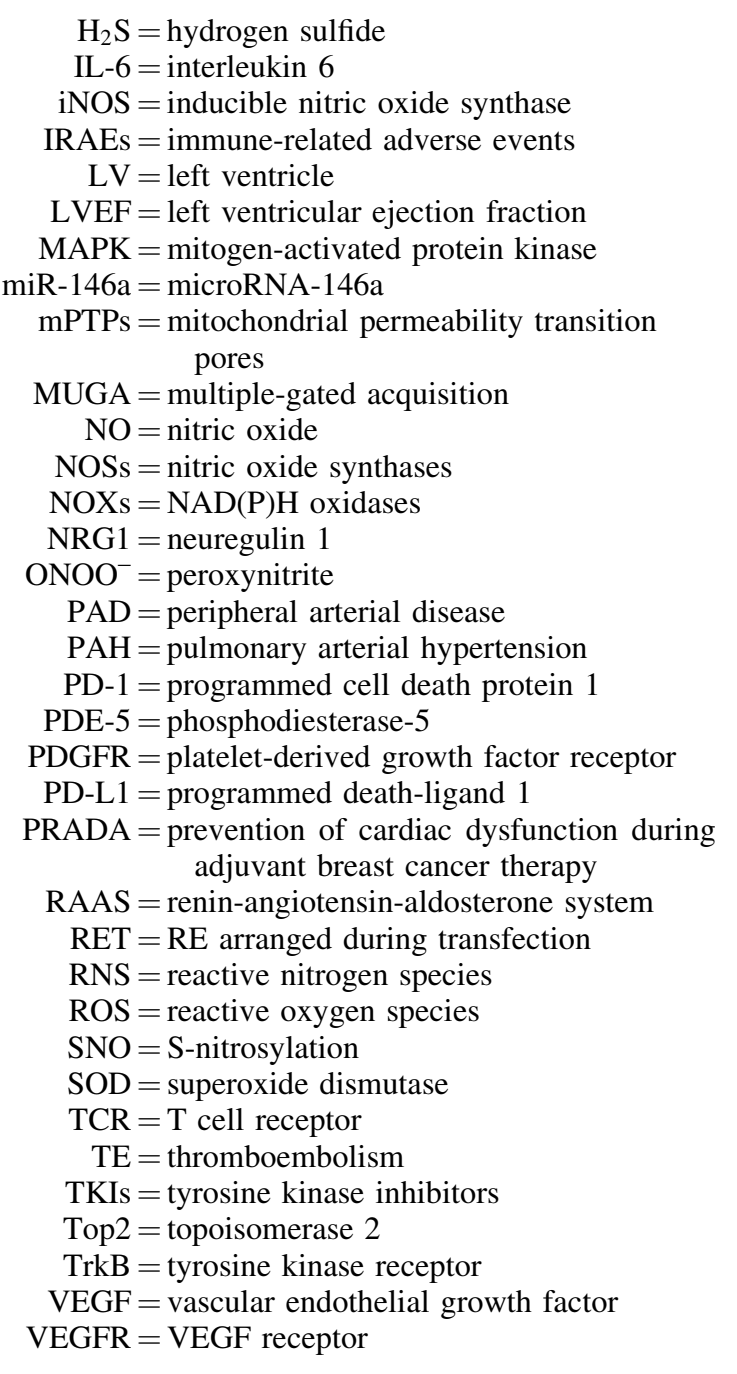

